A DYNAMIC MODEL FOR AIRCRAFT POSTSTALL DEPARTURE,

\author{
by \\ Mark Andrew "Hreha/l \\ Dissertation submitted to the Faculty of the \\ Virginia Polytechnic Institute and State University \\ in partial fulfillment of the requirements for the degree of \\ DOCTOR OF PHILOSOPHY \\ in \\ Aerospace and Ocean Engineering
}

APPROVED:

$\rho \overline{\text { F. H. LUTZE, CHAIRMAN }}$

E.-M. ELIFF

角. J. KELLEY

J. F. MARCHMAN

J. A. BURNS

May 1982

Blacksburg, Virginia 


\section{ACKNOWLEDGEMENTS}

The author wishes to express his appreciation to Professor Frederick H. Lutze, Chairman of the Committee, for his direction and encouragement in the development of the research and the successfur completion of the study.

He is grateful to Professor E. M. Cliff for his prompt and critical review of the paper and his suggestions for improvement.

The author would like to thank Professor J. F. Marchman, Professor J. A. Burns and Professor H. J. Kelley for valuable technical and mathematical guidance. 


\section{DEDICATION}

To my mother and father

They supported me in every phase of this research effort with encouragement and inspiration. 
TABLE OF CONTENTS

Page

TITLE .......................... $i$

ACKNOWLEDGEMENTS ........................ $i$

DEDICATION ..............................

TABLE OF CONTENTS ........................ iv

LISTS OF TABLES AND FIGURES .................. . . . vi

SYMBOLS ................... ix

Chapter

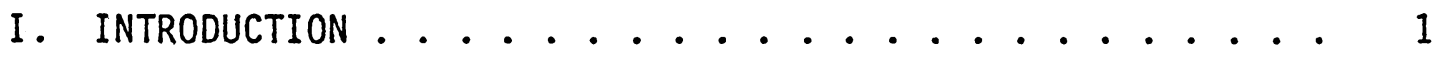

I.1 Poststall Flight Phenomena .......... 4

I.2 Purpose for Conducting Poststall Research . . . . 7

I.3 Proposal of Dynamic Model . . . . . . . . . 9

II. STALL/SPIN INVESTIGATIVE METHODS ........... 15

II.1 Full Scale Flight Testing .......... 15

II.2 Scale Model Stall/Spin Testing ......... 16

II.3 Analytical Stall/Spin Techniques ........ 21

III. PRESENTATION OF DYNAMIC MODEL ........... 25

III.I Coordinate systems ........... 25

III.2 Vortex System . . . . . . . . . . . . 28

III.3 Nonlinear Lifting Line Procedure (Levinsky) . . . . 31

III.4 Aerodynamics/Simulation Integration ..... 34

IV. PRESENTATION OF RESULTS ........................ 38

IV.1 Lift Curve Characteristics ......... 38

IV.2 Analytical Forced-Roll Oscillation Data ..... 41

IV.3 Six Degree-of-Freedom Flight Simulations ..... 43

V. DISCUSSION OF RESULTS ................ 46

V.1 Forced Oscillation Data ........... 46

V.2 Stall Departure Flight Simulations ...... 50 
Chapter

Page

VI. CONCLUSIONS AND RECOMMENDATIONS . . . . . . . . . . . 59

REFERENCES . . . . . . . . . . . . . . . . . . 65

TABLES . . . . . . . . . . . . . . . . . . . 69

FIGURES . . . . . . . . . . . . . . . . . . 72

APPENDIX A EQUATIONS OF MOTIONS . . . . . . . . . . . . 101

APPENDIX B AXES TRANSFORMATIONS . . . . . . . . . . . . 103

APPENDIX C LOCAL ANGLE OF ATTACK DEFINITION AND FORCE RESOLUTION. 106

APPENDIX D DOWNWASH CALCULATIONS OF VORTEX SYSTEM . . . . . . • . 111

APPENDIX E FORCED-ROLL OSCILLATION DATA REDUCTION . . . . . . . 117

VITA ............................ 119 


\section{LIST OF TABLES}

Table

Page

1 AIRCRAFT MASS AND GEOMETRY CHARACTERISTICS . . . . . 70

LIST OF FIGURES

Figure

1 AIRCRAFT GEOMETRIC MODEL ............ 73

2 BODY - AXIS SYSTEM ................. 74

3 INITIAL VORTEX SYSTEM GEOMETRY .......... 75

4 GEOMETRIC RELATIONSHIP BETWEEN CONTROL POINT $i$ AND PARALLELOGRAM LATTICE ELEMENT $j, k \ldots . . . . . . .76$

5 DYNAMIC MODEL FLOWCHART ............ 77

6 LIFT CURVE FOR BASIC $64_{2}-415$ AIRFOIL SECTION . . . . . 78

7 LIFT CURVE FOR $65_{1}-012$ AIRFOIL SECTION . . . . . . 79

8 LIFT CURVE FOR $64_{2}-415$ AIRFOIL SECTION WITH DROOPED

LEADING EDGE ................. 80

9 INPUT AND OUTPUT SIGNALS FOR FORCED-ROLL OSCILLATION

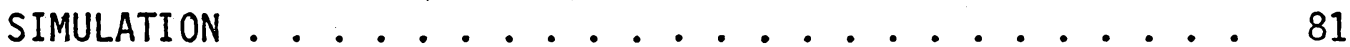

10 OUTPUT SIGNAL FOR FORCED-ROLL OSCILLATION SIMULATION $\theta=14^{\circ} \ldots \ldots . \ldots . \ldots 2$

11 OUTPUT SIGNAL FOR FORCED-ROLL OSCILLATION SIMULATION $\theta=18^{\circ} \ldots \ldots . \ldots . \ldots . \ldots 3$

12.a DAMPING-IN-ROLL PARAMETER vS ANGLE OF ATTACK, BASIC WING . 84

12.b DAMPING-IN-ROLL PARAMETER vS ANGLE OF ATTACK, DROOPED OUTBOARD SEGMENTED WING 
13 PHYSICAL AND COMPUTED STALL CELL PATTERNS . . . . . . . 85

14 SYMMETRIC STALL PENETRATION, BASIC WING - LONGITUDINAL PARAMETERS

15.a SYMMETRIC STALL PENETRATION, BASIC WING - LONGITUDINAL PARAMETERS, ASYMMETRIC INDUCED ANGLE-OF-ATTACK SOLUTION .

15.b SYMMETRIC STALL PENETRATION, BASIC WING - LATERAL PARAMETERS, ASYMMETRIC INDUCED ANGLE-OF-ATTACK SOLUTION • 88

15. C SYMMETRIC STALL PENETRATION, BASIC WING - ALTITUDE VS CROSS RANGE PLOT, ASYMMETRIC INDUCED ANGLE-OF-ATTACK SOLUTION

16.a ASYMMETRIC STALL PENETRATION, BASIC WING - LONGITUDINAL PARAMETERS ................... 90

16.b ASYMMETRIC STALL PENETRATION, BASIC WING - LATERAL PARAMETERS

16.C ASYMMETRIC STALL PENETRATION, BASIC WING - ALTITUDE VS CROSS RANGE PLOT . . . . . . . . . . . . . . . 92

17.a SYMMETRIC STALL PENETRATION, OUTBOARD DROOPED LEADING EDGE - LONGITUDINAL PARAMETERS, ASYMMETRIC INDUCED ANGLE-OF-ATTACK SOLUTION

17.b SYMMETRIC STALL PENETRATION, OUTBOARD DROOPED LEADING EDGE - LATERAL PARAMETERS, ASYMMETRIC INDUCED ANGLEOF-ATTACK SOLUTION

C.1 LOCAL WING STATION $i$ GEOMETRY FOR DETERMINING ANGLES OF ATTACK

C.2 GEOMETRY FOR COMPUTING LOCAL FORCE COEFFICIENT $\vec{C}_{F i}$ IN TERMS OF BODY-AXIS SYSTEM VECTORS . . . . . . . . . . 96

D.1 GEOMETRY USED IN DERIVING THE VELOCITY INDUCED AT POINT CP BY A STRAIGHT VORTEX FILAMENT . . . . . . .

D.2 VECTOR RELATIONSHIP BETWEEN A VORTEX ELEMENT $j$ AND CONTROL POINT $i$ IN INERTIAL SPACE . . . . . . . . . 
viii

Figure

Page

D.3 INERTIAL SPACE PLANE GEOMETRY FOR COMPUTING THE SCALAR $h$. . . . . . . . . . . . . . . . . 99

D.4 INERTIAL SPACE GEOMETRY FOR DETERMINING THE LOCAL-WING NORMAL $\hat{n}_{j}$..................... 100 
The longitudinal and lateral aerodynamic characteristics presented in this paper are referred to the body-axis system as shown in Figure 2. Forces and moments are reduced to standard coefficient form on the basis of aircraft geometric properties. Dimensional quantities are given in both the International System of Units (SI) and U.S. Customary Units. Calculations were made in the U.S. Customary Units.

Coefficients and symbols used here are defined as follows:

b wing span, $m(f t)$

c aerodynamic chord, $m(f t)$

$\overrightarrow{\mathrm{C}}_{\mathrm{F}}$ force coefficient vector, $\vec{F} / \bar{q} S$

$C_{L}$ two-dimensional lift coefficient, $L / \overline{q C}$

$c_{\ell}$ rolling-moment coefficient, $M_{\ell} / \bar{q} S b$ pitching-moment coefficient, $M / \bar{q} S C$

$C_{N}$ yawing-moment coefficient, $\quad N / \bar{q} S b$

$c_{x}$ axial force coefficient, $x / \bar{q} S$

$c_{Y}$ side force coefficient, $Y / \bar{q} S$

$C_{Z} \quad$ normal force coefficient, $Z / \bar{q} S$ 
$\vec{F}$

$r$ force vector, $N\left(1 b_{f}\right)$ gravitational acceleration, $\mathrm{m} / \mathrm{sec}^{2}\left(\mathrm{ft} / \mathrm{sec}^{2}\right)$ moment of inertia about $x$-axis, $k g-m^{2}\left(s l-f t^{2}\right)$ moment of inertia about $y$-axis, $k g-m^{2}\left(s 1-f t^{2}\right)$ moment of inertia about $z$-axis, $\mathrm{kg}-\mathrm{m}^{2}\left(\mathrm{sl}-\mathrm{ft} \mathrm{t}^{2}\right)$ lift force per unit span, $\mathrm{N} / \mathrm{m}\left(1 \mathrm{~b}_{f} / \mathrm{ft}\right)$ aircraft mass, $\mathrm{kg}(\mathrm{sl})$. pitching moment, $m-N\left(f t-1 b_{f}\right)$ rolling moment, $m-N\left(f t-1 b_{f}\right)$ yawing moment, $\quad m-N\left(f t-1 b_{f}\right)$ roll $\mathrm{rate}, \mathrm{rad} / \mathrm{sec}$ angular acceleration in $\mathrm{roll}, \mathrm{rad} / \mathrm{sec}^{2}$ pitch rate, $\mathrm{rad} / \mathrm{sec}$ angular acceleration in pitch, $\mathrm{rad} / \mathrm{sec}^{2}$ dynamic pressure, $\quad \mathrm{N} / \mathrm{m}^{2}\left(1 \mathrm{~b}_{\mathrm{f}} / \mathrm{ft}^{2}\right)$ yaw rate, $\mathrm{rad} / \mathrm{sec}$ angular acceleration in yaw, $\mathrm{rad} / \mathrm{sec}^{2}$ 


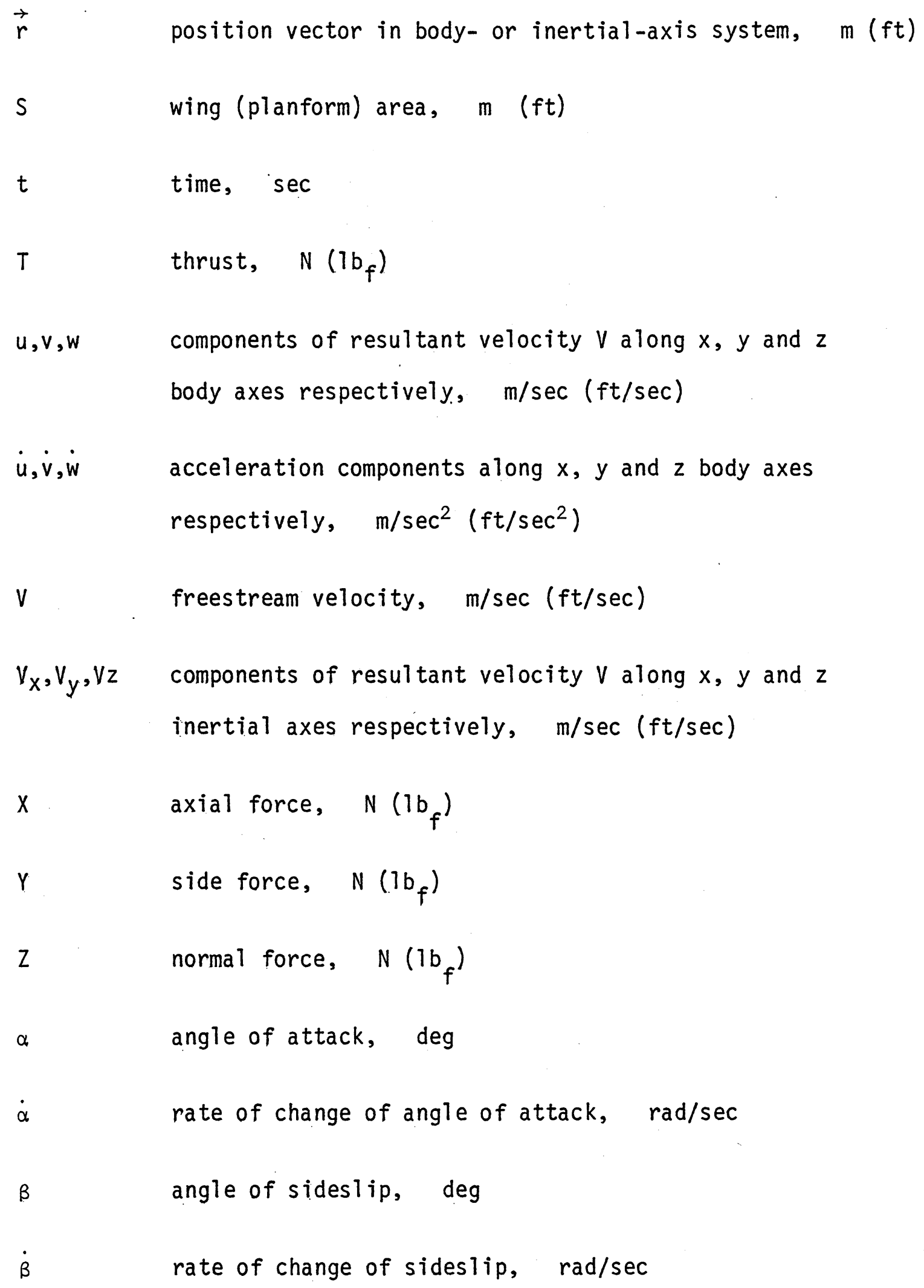




\begin{tabular}{|c|c|}
\hline$\Gamma$ & circulation strength, $\mathrm{m}^{2} / \mathrm{sec}\left(\mathrm{ft}^{2} / \mathrm{sec}\right)$ \\
\hline$\Lambda$ & lifting surface sweep angle, deg \\
\hline$\eta$ & lifting surface dihedral angle, deg \\
\hline$\nu$ & lifting surface incidence angle, deg \\
\hline$\phi$ & Euler bank angle, deg \\
\hline$\psi$ & Euler yaw angle, deg \\
\hline$\theta$ & Euler pitch angle, deg \\
\hline$\rho$ & atmospheric density, $\quad \mathrm{kg} / \mathrm{m}^{3}\left(\mathrm{sl} / \mathrm{ft}^{3}\right)$ \\
\hline$\mu$ & viscosity, $\quad \mathrm{N}-\mathrm{sec} / \mathrm{m}^{2}(\mathrm{~s} 1 / \mathrm{sec}-\mathrm{ft})$ \\
\hline$\omega$ & oscillation frequency, \\
\hline$\omega$ & vehicle angular rate, \\
\hline$\Delta \mathrm{W}$ & downwash velocity, $\mathrm{m} / \mathrm{sec}(\mathrm{ft} / \mathrm{sec})$ \\
\hline
\end{tabular}

SUBSCRIPTS

cg

center of gravity

b

body axis system

1

local (wing) axis system 
$x i i i$

$p$

pitch (geometric)

h

horizontal axis system

d

induced

eff

effective

MAX

maximum amplitude value

OUT

out-of-phase component

$\infty$

freestream condition

20

two dimensional

$3 D$

three dimensional 


\section{CHAPTER I}

\section{INTRODUCTION}

Aircraft "stall/spin problems represent one of the last technical frontiers in the aviation field and have significant impact on (especially) the general aviation industry" (Ref. 1). This observation was made in a concluding review of several research programs presented at the General Aviation Stall/Spin Workshop held at Langley Research Center on September 3 and 4, 1980. Citing a general insufficient data base in the stall/spin area, a representative group from the aviation community suggested improved flight and wind tunnel testing. The development of analytical techniques designed for the investigation of the stall and flight path departure was particularly emphasized. Special note was made that an ultimate goal of this high angle-ofattack research might be the development of an engineering handbook or "cookbook" to be used as a design phase aid for stall/spin prediction. The objective of the present research is to propose an engineering model applicable to such analysis of aircraft poststall flight characteristics.

The poststall dynamics addressed in the present paper may be described as uncontrollable motions characterized by large rolling and yawing rates. The loss of lateral stability and controllability subsequent to the stall is due to the tendency of unswept wings to experience unstable damping in roll and autorotation near stall (Ref. 2). 
The resulting departure from the intended flight path has in general been observed to occur as either an abrupt roll-off, the wing drop, or divergent yawing and rolling oscillations, wing rock, which generally terminate in a violent yaw excursion called nose slice. An obvious consequence of such departures is the development of high sink rates and associated rapid loss of altitude. The present dynamic model is constructed with the specific capability of analyzing and predicting such poststall phenomena.

Fundamental to the analysis of all atmospheric flight mechanics is the interaction of aerodynamics with vehicle dynamics. The direct influence of a vehicle's dynamic parameters, i.e., its state, on the aerodynamic forces and moments is illustrated by the familiar variations of lift, drag and pitch moment with angle of attack. This is an example of indicating the dependence of longitudinal aerodynamics on longitudinal variables. A direct analog exists in the lateral case in that side force and rolling and yawing moments are usually represented as functions of sideslip angle. While such pairing of aerodynamics with associated state variables is appropriate for modeling conventional, low angle-of-attack flight regimes, the complex nature of poststall departure mechanics demands the development of more sophiscated models. Recent studies of high angle-of-attack aerodynamic modeling have shown the importance of proper aerodynamic representation in the prediction of poststall flight motions (Ref. 3 and 4). Such representation may include among other considerations aircraft angular rate 
dependence and cross-coupling influences, for instance, roll moment due to angle of attack. Perhaps the conservative approach of retaining the influence of the complete physical state on all forces and moments is most appropriate, at least until further experience with analytical stall techniques indicates al ternative models.

The converse relationship, that the aerodynamic forces govern the dynamics of the aircraft, is directly observable from the Newtonian formulation of the equations of motion. Indeed, the axial, normal and side forces and the roll, yaw and pitch moments can be considered forcing functions to the dynamic equations. (See Eqs. A1-A6.) The present model preserves this interaction of aerodynamics and vehicle dynamics by incorporating an appropriate aerodynamics generating package used interactively with full six degree-of-freedom flight simulation. Hence, given an initial flight condition and a predetermined sequence of control inputs, the forces and moments are computed using a nonlinear lifting line method based on the current vehicle state and then used in a single trajectory integration step. For the next step, the aerodynamics are calculated with respect to the updated dynamic parameters and the process continues until the specified flight time has elapsed. This aerodynamics/simulation procedure reflects the force/dynamics interaction with explicit complete state influence and the resulting trajectory provides a quantitative description of the flight, in this case departure, mechanics. 


\section{I.1 Poststall Flight Phenomena}

The problem of poststall flight analysis can be characterized by four distinct phenomena: (1) nonlinear aerodynamics, (2) unsteady aerodynamics, (3) three dimensional effects and (4) vehicle configuration dependence. It is proposed that a valid analytical departure model must be capable of exploiting the effects of these observable physical characteristics. Justification for requiring these elements in a plausible analytical technique is briefly outlined below while the actual mechanics of introducing them into the computations is presented in Chapter III.

As a two-dimensional subsonic airfoil approaches and penetrates the stall, the nonlinear aerodynamics generally appear as either a rounded section lift curve with a large negative poststall slope (trailing edge sta11) or an abrupt curve discontinuity subsequent to stall (leading edge sta11). Trailing edge stall is associated with gradual turbulent separation propagating upstream from the rear of the airfoil. A short bubble located at an airfoil's leading edge initiates the onset of leading edge stall (Ref. 5). Separated-flow lifting properties of either type of stall are generally characterized by a positive lift curve slope. The three factors determining where and when flow separation occurs are boundary layer profile and thickness and adversity of the local pressure gradient. Al1 three factors are determined by the pressure distribution, the first two carrying the effects of the pressure gradient on boundary layer development up to the separation point while the third affects flow separation in a very direct (local) manner (Ref. 6). While several 
separation flow models are being developed from consideration of these physical fluid properties (Ref. 6 and 7), the present technique bypasses the complexities of simulating the stall mechanism in favor of modeling the effects of the nonlinear phenomenon.

The most notable evidence of unsteady aerodynamic effects are the dynamic (hysteresis) loops which occur in the normal force and pitching moment curves for a 2D airfoil oscillating in pitch near stall angle of attack (Ref. 8). The shape of these loops has been shown to be strongly dependent on oscillation frequency further emphasizing the importance of time effects. Associated with dynamic penetration of the stall are an overshoot of the static stall and undershoot of static reattachment loads (Ref. 6). Since the overshoot and undershoot are mainly results of dynamic effects on the boundary layer, they are not predicted by the modified lifting line theory employed in the present model. However, the utilization of a finite element, unsteady wake as presented in Chapter III allows for the reproduction of reasonable lift hysteres is Toops.

The highly dynamic trajectory of an aircraft undergoing poststall flight path departure immediately suggests the inadequacy of a 1 inear wake geometry for a vortex-lattice procedure. Instead, three-dimensional aerodynamic effects may be accounted for by adopting a wake geometry logic originally developed and applied to the analysis of helicopter rotors and flexible wings. This technique allows for the shedding of vortex elements into the stream surface in which they originate. Distortion 
and convection of the wake due to the mutual interactions of the shed vortices are ignored in this model. However, since the streamwise dimension of the wake used in calculating wing loadings is small (about two fuselage lengths), a reasonable assumption is that any effects on load computation due to wake distortion are negligible.

Perhaps the greatest obstacle affecting the development of a generalized stall/spin analytical technique is the strong influence of an aircraft's configuration on its poststall flight characteristics. Examples of configuration dependence can be found in NASA's experience with the flight testing of single-engine general aviation aircraft. One low-wing aircraft, the Grumman American AA-1 Yankee, exhibits two spin modes: one moderately steep ( $60^{\circ}-90^{\circ}$ pitch down attitude) and the other moderately flat $\left(20^{\circ}-60^{\circ}\right.$ attitude). The aircraft readily enters and satisfactorily recovers from the steep spin while a specific sequence of elevator inputs is required to transition to the unrecoverable flat mode. The Beechcraft $\mathrm{C}-23$ Sundowner is also a low-wing configuration but will not spin unless ailerons are deliberately deflected against the spin during entry. Recovery from the moderately steep spin mode is performed quickly using standard recovery controls. A high-wing airplane, the Cessna 172 Skyhawk, has a very steep spin characterized by a high sink speed and a high number of turns to stabilize. Automatic recovery into the spiral is often accomplished even with pro-spin controls held. Anti-spin controls effect a very quick spin exit. Further evidence of the strong influence of vehicle configuration may be found 
in Reference 10 where the effects of tail configuration, wing leadingedge modifications, moment-of-inertia variations, fuselage modifications and center-of-gravity location on the spinning characteristics of the Yankee aircraft are presented.

The approach taken in the present research allows for the particular definition of a given aircraft geometry by mathematically representing a11 lifting surfaces with discrete systems of bound vortex segments and their associated control points. This mathematical model respects not only the spacial relationships of the lifting surfaces, but also their individual orientations with respect to a body-axis system. No attempt is made to model fuselage effects. It is recognized that fuselage-flow interactions may significantly alter quantitative results but qualitative explanations inferred from the wing-empennage model simulations will still be valid.

\section{I.2 Purpose for Conducting Poststal1 Research}

As mentioned in the opening paragraph of this Introduction, this research has been motivated by the suggestions of representatives from different segments of the general aviation industry who indicated the desirability of poststall analytical prediction techniques. Opening comments at NASA's 1980 Stall/Spin Workshop conceded that "the lack of validated analytical tools, together with many unsuccessful attempts to generalize results from one configuration test to another has added a considerable amount of testing to (Langley's) original planned program and lessened the number of stall/spin experts both at this center and 
elsewhere" (Ref. 11). Emphasizing the need for predictive models, several speakers encouraged the development of "analytical tools that are very much needed in the design process." Direct application of such poststall models may assist in the much requested "research on advanced or unconventional configurations with the objective of obtaining passive or purely aerodynamic methods for preventing the stall departure and spin" (Ref. 1). The ability of the present dynamic model to include effects of the four previously outlined stalling phenomena, especially the aerodynamic configuration dependence, make it a strong candidate for a design and analysis-oriented tool.

The aircraft industry's concern in poststall flight characteristics stems from statistics confirming that stalling and spinning are major causal factors in fatal general aviation accidents. Stall and spin accidents presently account for about $28 \%$ of a 11 general aviation fatalities. This is a significant reduction from the $50 \%$ figure recorded for the postwar period 1945-48. Indeed, further analysis of older aircraft designs examined for their stall and spin accident records in modern flying situations indicates the stall/spin fatality rate is strongly related to aircraft design. However, the percentage of stall/spin accidents has remained fairly constant over the last one and one-half decades (Ref. 12). Increased analytical research in stall/ poststall flight dynamics may again accelerate the improvement in fatality rate through configuration design. Reference 12 also lists the recently computed percentage breakdown of stall/spin accidents by 
phase of flight. Roughly one fourth of these accidents occur in either the takeoff phase (extending from the start of takeoff roll to normal climbout) or the landing phase (beginning with entry to the traffic pattern and extending to touchdown or go-around). Of the remaining accidents occurring in the "inflight" phase, $85 \%$ of the inadvertent stalls appear initiated by accelerated stall maneuvers (turning and/or climbing) in which the pilot was unaware of the aircraft's high angle of attack. The purpose for presenting these statistics is to illustrate that major accident causing maneuvers may be "flown" with the proposed dynamic model subsequent to aircraft production or even initial flight testing and the resulting uncontrollable departures carefully studied.

\section{I.3 Proposal of Dynamic Model}

Following is a brief proposal of the dynamic model developed in the present research effort for the analysis of poststall departures. The mathematical description and details are presented in Chapter III. As indicated previously, the model is composed of an aerodynamic package used interactively with a six degree-of-freedom digital motion simulator. The computational aerodynamics are due to a nonlinear lifting line procedure with unsteady wake effects developed for the prediction of wing span loadings by E. S. Levinsky (Ref. 13). This procedure was specifically proposed for simulating and alleviating adverse wing stalling characteristics such as wing rock and wing drop. The idea of using nonlinear section lift data in a lifting line technique in order to model the stall is not new; for example, Reference 14 contains a linear 
wake theory useful in computing static wing lift forces near the stall. Several factors should be considered in determining the validity of extending classical lifting line theory into the region of stalled and partly stalled wings. Since the present model does not use a linear streamwise wake geometry nor any small angle approximations, the major assumption in jeopardy is that of two-dimensional flow at each spanwise station. It is recognized that the section lift curve may not be transferable with complete accuracy from two- to three-dimensional flow. Sti1l, the concept of circulation and the Kutta-Joukowski formula have been shown extensible to flows with viscous wakes. Hence, the qualitative relationships between low angle-of-attack and stall applications must remain the same (Ref. 15). In fact, a nonlinear lifting line (linear wake) approach was applied to departure and spin entry wing span load calculations in Reference 5; however, that model was not incorporated with fully configured vehicle dynamic simulation. The method used in the present proposal follows closely the theory of Levinsky except for the exclusion of body effects and a modification to the wake vortex system allowing a nonlinear geometry.

The nonlinear lifting line formulation provides for the representation of each lifting surface by a system of finite bound vortex elements and associated control points. Each vortex segment, or panel, is assumed to act aerodynamically (including stall) like a 2D airfoil in steady flow at an effective angle of attack which depends on the aircraft's dynamics. This hybrid lifting line/wind tunnel data method makes use of 
experimental 2D section lift data to compute each panel's force magnitude and thus the total span load distribution. Unsteady effects are introduced through the wakes which are composed of parallelogram lattice elements formed from the streamwise and transverse vortices shed from each panel. The parallelogram elements are fixed in space to the stream surface in which they originate. The transverse vorticity is collected and shed discretely as the aircraft moves through space; hence, the strengths of these vortices may vary in time while the velocities they induce at the control points vary with distance. The modeling employed here is similar in nature to that used in the case of helicopters and has also been applied in formulating an unsteady lifting line theory of flapping wings for the analysis of the forward flight of birds (Ref. 16).

The dominating effects of a given aircraft configuration on departure dynamics are included by mathematically reconstructing the vehicle's lifting surfaces. A system of bound vortex elements is classically placed at the $25 \%$ chord location of the main wing, horizontal tail and vertical fin and respective control points at $75 \%$ of the chord streamwise. Variables specifying the spacial relationships of the configuration are longitudinal position of the wing with respect to the center of gravity, longitudinal and vertical positions of the horizontal tail and vertical fin, and the spanwise dimension of each lifting surface. The orientations of the wing and horizontal tail with respect to a body-fixed axis system are governed by variable pitch incidence, dihedral and sweep while only sweep angle need be specified for the fin. The taper of each 
surface may be fixed through a vector containing spanwise chord length information. Finally, the influence of a particular airfoil section is accounted for by inserting the proper 2D lift curve data into the lifting line theory. An application illustrating the flexibility of this construction is the modeling of a wing with multiple airfoil profile sections by using the appropriate lift curves at the desired panel stations. This enables, for instance, the investigation of departure benefits due to passive spin entry resistance methods such as outboard drooped leading edge wing modifications. Once the aircraft configuration is modeled, full scale mass and inertia properties are imparted to it and it is "flown" through space by integrating the rigid body equations of motion subject to the forces calculated with the nonlinear lifting line theory.

Results of the stall/poststall flight investigations obtained with the dynamic model include generation of forced-roll oscillation data, six degree-of-freedom simulation of several stalling maneuvers, effects of wing modifications on forced-roll data and poststall departure and the demonstration of multiple lifting line solutions. These multiple solutions were first identified by von Karman who noted the possibility of nonunique solutions to Prandtl's integro-differential equation if evaluated with negative lift curve slopes. Von Karman hypothesized that this phenomenon of nonunique solutions might also occur for lift versus angle of attack curves having portions of both positive and negative slopes, or having discontinuities. Results of calculations using a section lift curve exhibiting an abrupt stall discontinuity reported 
in Reference 15 substantiate von Karman's supposition. These results verify the mathematical existence of asymmetrical spanwise lift distributions and associated large rolling moments near the stall without postulating any lateral asymmetric flight condition such as rolling velocity or sideslip. Sears (Ref. 15) suggests that these asymmetric distributions may explain the violent rolling moments evident in wind tunnel testing of stalling symmetric wings firmly fixed to the balance.

The present research furthers this line of reasoning by presenting evidence that asymmetric lift distributions are sufficient to initiate poststall wing drop departures from symmetric flight conditions. The choice among the multiple solutions (either symmetric or asymmetric load distributions) probably depends on the relative stability of the corresponding circulation distribution due to small disturbances (Ref. 13). Although no formal stability analysis is presented here, it is proposed through example that the establishment of a particular solution may be inferred by examining the effect of small flight asymmetries (out-oftrim conditions) existing at the stall break.

The technique of generating forced-roll oscillation data described in this research is probably the first attempt at analytically producing the variation of the dynamic stability term $C_{L_{p}}+C_{L_{\dot{\beta}}}$ sin $\alpha$ with angle of attack. The results echo graphical trends and occurrence of sign change in the derivative term exhibited by wind tunnel data. This is particularly encouraging despite the crude discretized wing and wake lattice and the fact that no fine tuning of the system was attempted. 
This capability of the dynamic model may prove to be a powerful tool in the design-phase motion stability analysis of future aircraft concepts. 
STALL/SPIN INVESTIGATIVE METHODS

Comments of experienced stall/spin researchers quoted in the Introduction point out the overwhelming emphasis placed on experimental techniques in investigating high angle-of-attack flight characteristics. The development of analytical methods, especially predictive models useful in the design phase, is an area for research advancement strongly urged by industry representatives. This chapter is devoted to a brief review of current sta11/spin investigative techniques (both experimental and analytical). It is suggested that the complexities, costs and lack of generality of experimental work in this area together with less than adequate analytical methods confirm a critical need for stall departure models.

\section{II.1 Full Scale Flight Testing}

Foremost among flight test objectives is documentation of actual sta11/spin characteristics for a particular aircraft design. Such documentation usually takes the form of time histories of selected flight parameters (e.g. angle of attack, velocity, pitch attitude) sensed during the maneuver and telemetered from the test airplane to ground recording stations. The data recorded provide Reynolds number and other scale effects at altitude for model correlation. A unique advantage of full scale flight testing is the presence of a pilot who can perform the roles of a feedback data sensor and a human servo. The 
pilot's ability to sense pitch attitude and linear and angular accelerations permits efficient assignment of human factors significance to certain parameters. His inflight observations and post-flight opinions provide information on the effectiveness of specific control techniques.

However, the fact that a pilot is onboard an aircraft being maneuvered out of its controllable flight envelope complicates the testing procedure in that adequate pilot safety must be provided. Typical safety precautions include an airplane spin recovery parachute, pyrotechnics for emergency egress (door jettison), strict adherence to verbally reported check 1 ists and decision altitudes, and helicopter standby for spin chute retrieval and pilot rescue. In addition to the spin recovery systems, the test aircraft are experimentally modified with angleof-attack sensing wing tip booms, external cameras, internal instrumentation and communications and ballasting provisions. It is quite possible that the spin-test aircraft may have aerodynamic and mass properties different from the production model from which it was derived. (Ref. 17 and 18)

\section{II.2 Scale Model Sta11/Spin Testing}

Experimental poststall flight investigations via the use of scale models can be divided into three specific techniques: (1) flight trajectory simulation by radio controlled models, (2) spin mode identification using the Langley Spin Tunnel and (3) hybrid experimental/analytical spin mode analysis using a rotary balance rig. The objectives of each procedure are outlined below along with descriptions of the testing 
technique, necessary hardware and facilities, and results obtained.

The basic objective of radio-controlled model testing is to provide a low-cost flight-test technique. Thus far, the main approach has been aimed at a qualitative assessment of general sta11, spin entry, steady spin and recovery characteristics. Ultimately, an instrumented test setup would be composed of a model command radio-control link, tape recorded comments from ground-based pilot and observer, test documentation via movie camera and a radio-controlled downlink telemetry system which is time-correlated with the motion picture film. Seven sensors onboard the model measure and relay the values of angle of attack and sideslip on each wing tip and the positions of the three control surfaces (Ref. 19). The models are typically scaled to $1 / 5$ full size and weigh between 12 and 16 pounds. These research models are equipped with spin recovery parachute systems (Ref. 20).

Radio-controlled scale models have proved good simulators of general stall and spin entry characteristics. They are capable of displaying basic poststall motions such as wing drop departure and transition into steady spin. In the case of the Yankee aircraft (Ref. 20), the model data provided good correlation with full scale flight tests, despite unresolved problems with scale effects. In addition, the pilot was able to apply control sequencing similar to full scale experience which forced the model from its moderately steep spin mode into a flatter mode. The analysis of configuration dependence must, of course, be handled by the construction of multiple models. 
In the spin tunnel testing technique, dynamic aircraft models are hand-launched into the vertical rising air stream of the spin tunnel with initial rotation and at steep (or flat) attitudes. The model then seeks an equilibrium spin mode where such data as pitch attitude (with respect to the horizon) and rotation rate are recorded. General aviation model scales vary from $1 / 10$ to $1 / 15$ full size. The ratio of gravitational to inertial forces for the airplane is preserved in the tests by dynamically scaling the spin models according to the Froude number $\left(\mathrm{V}^{2} / \mathrm{gc}\right)$. Hence, the significant parameters can be measured during the spin test and converted to full-scale values.

The spin tunnel test is useful in identifying stable developed spin modes and determining parametric effects such as center-of-gravity location and mass changes. Spin and recovery characteristics may be obtained for various combinations of rudder, elevator and aileron deflections. The spin tunnel has also been applied to the problem of sizing emergency spin recovery parachutes and their risers (1ength of line from canopy suspension lines to aircraft). If the models are equipped with radiocontrolled servos actuating the control surfaces, then the possibilities of effecting a recovery from a developed spin through some sequence of control inputs can also be investigated.

Application of the spin tunnel technique to general aviation aircraft has not been as successful as when used on military fighter type aircraft. Reference 21 suggests that the discrepancy is due to Reynolds number. This similarity parameter cannot be simultaneously scaled with Froude number without changing the fluid to change the kinematic 
viscosity $\mu / \rho$ (Ref. 22). Reynolds number effects become more important in the general aviation case where the spinning characteristics are affected to a much larger extent by wing and airfoil shape rather than configuration. Indicative of this scaling problem are results of tests on the Yankee aircraft wherein the spin tunnel predicted two spin modes (one moderately flat and the other moderately steep) which correspond roughly to the modes exhibited by the full scale aircraft. However, the spin tunnel was unable to predict the degraded spin performance of the airplane when both it and the scaled model were modified by drooping the leading edges (Ref. 21). Finally, this experimental technique has no provision for analyzing the stall departure or spin entry.

The rotary-balance technique is a hybrid experimental/analytical procedure developed for the purpose of identifying an airplane's aerodynamic characteristics in a rotational flow environment. The facility includes a 6 component balance through which a model is mounted to a rotary rig located in the 20 -foot spin tunnel at Langley Research Center. The influence of pitch attitude, bank attitude and rotation rate on the aerodynamic forces and moments of a simulated spinning vehicle can be measured with this set-up. The procedure was devised with primary objectives being the acquisition of a large aircraft spin research data base, provision of required data for the analysis of aircraft spin and the method's utilization as a design tool for configuration definition. A typical data set produced by the rotary-balance technique consists of graphical presentation of each of the six aerodynamic components versus nondimensional rotation rate with pitch and bank attitudes as parameters. 
The effects of control settings can also be measured by deflecting the models control surfaces.

The rotary rig has been coupled with an on-line data acquisition and reduction system for the identification of steady spin modes. The technique requires satisfying the moment equations resulting from balancing the aerodynamic moment with the inertial moment simultaneously in a11 three planes (pitch, rol1 and yaw). A computer program was generated that first satisfies pitch equilibrium by locating rotation rates for which the aerodynamic and inertial moments are equal at selected angles of attack. The result is a pitch-equilibrium relationship between alpha and spin rate. Combinations of these two parameters are then used in the rolling moment equation to solve for the sideslip angle, or effectively the bank attitude, necessary for roll moment balancing. The final step involves searching the data for yaw moment equilibrium at the prescribed angle of attack, bank attitude and spin rate combinations (Ref. 23).

When applied to the Yankee configuration, this experimental/analytical identification of steady-state spin modes yielded results which correlated very well with the free spin tunnel data discussed above. The method was able to verify the existence of both the moderately flat and moderately steep spin attitudes. The fact that both investigative techniques use the same spin tunnel facility and very similarly sized models may bear on the excellent agreement between the results. This implies that the rotary-balance test is subject to the same Reynolds scaling difficulties encountered with the free spin test. Another 
similarity of the two procedures is evident in that the hybrid analysis is inapplicable to departure and spin entry flight.

\section{II.3 Analytical Stall/Spin Techniques}

The whole of analytical techniques currently available for the stall/ spin area can be divided into the linear analysis of equilibrium flight conditions and flight trajectory simulation. The aerodynamic modeling may be of experimental test origin or computational. Here, any procedure using reduced wind tunnel data but completely independent of interactive wind tunnel usage is considered to be purely analytical. Linear analysis can be further partitioned according to the method by which steady-state flight conditions are determined. For example, Reference 24 describes a numerical optimization technique (Davidon-Fletcher-Powe11) which minimizes a cost function defined as the sum of the squares of the state time derivatives by a variation of the steepest descent method. This method provides a systematic search which is capable of locating both stable and unstable equilibrium spin points. Another equilibrium identifier resembling the rotary-balance procedure in using graphical presentation of moment equilibrium conditions over a range of aircraft attitudes is given in Reference 25. In fact, this technique makes use of dynamic balance test data obtained with the rotary rig.

Linear analysis of equilibrium spin conditions is an outgrowth of small perturbation theory applied to straight and level flight. However, care must be taken for the spin case in that the nominal equilibrium point will in general have nonnegligible angular rates and sideslip 
angle in addition to large angle of attack. A detailed development of a general system matrix applicable to "nontrivial" flight conditions is presented in Reference 26. Eigen-analysis of the linearized system provides not only information on the stability of the identified spin modes but also indicates the sensitivity of motion characteristics with respect to aerodynamic modeling. In Reference 3, for example, three different aerodynamic data sets were examined for their effect on the prediction of poststall gyrations of the $F 5$ fighter aircraft. Results indicated the importance of isolating the acceleration stability derivatives from the forced oscillation data (e.g. $C_{L_{\dot{\beta}}}$ separated from the term $C_{L_{p}}+$ $C_{L_{\dot{\beta}}} \sin \alpha$ ) especially for the case of large sideslip. The linear analysis also provided quantitative explanation of the pilot-observed wing rock/yaw oscillation motions subsequent to the stall.

In an attempt to capitalize on the assumption that the rotarybalance data contains the necessary aerodynamic information to describe a steady spin, a substantial effort was made to interface this data base with the analysis of References 24 and 26. The rotary data used are for the baseline Yankee configuration (Ref. 27 and 28). The six component data, as discussed previously, are given in three-dimensional graphical form, the parameters being pitch and bank attitude and rotation rate. However, it is desired to have the dependency of the aerodynamic forces and moments expressed with respect to the complete state: alpha, sideslip, pitch, roll and yaw angular rates. The parameter transformations were formulated from the kinematics of the rotary motion, but, of course, once any three of the state variables were specified, the remaining two were 
automatically determined. Results similar to those reported in Reference 25 were obtained. A moderately flat spin mode of $72^{\circ}$ angle of attack and nondimensional spin rate $(\omega b / 2 V)$ equal to 0.56 was identified as well as a steeper mode with alpha equal to $27^{\circ}$ and a spin rate of 0.12 . However, a fundamental deficiency in data representation severely limited the usefulness of this method. The rotary data do not reflect the proper "dimensionality" necessary for a methodical search of the aerodynamics to locate possible spin equilibrium candidates nor can it be extended in its present form to do so. It is recommended that future rotary rig testing include the parameters spin radius and yaw orientation (with respect to the rig rotor arm) in addition to the three currently used. (Presently, for steep pitch attitudes, $0^{\circ}$ to $30^{\circ}$ measured from the vertical, data are obtained with the model mounted on a nonzero radius arm from the rotation axis, while flatter attitude data, $30^{\circ}$ through $90^{\circ}$, are measured with the model positioned on the spin axis. This does not constitute explicit representation of spin radius dependence.)

Application of the linear analysis to departure dynamics supposes that a steady-state flight condition can be found describing the motion. If such equilibriums exist for a particular aircraft, it is suggested that the highly dynamic nature of the poststall maneuver necessitates a logical, systematic search for their "locations" such as that outlined in Reference 24.

The general discouragement with the utility of existing analytical techniques led to the formulation of the dynamic model introduced in 
Chapter I. The model is one of the few flight trajectory simulation types of analytical tools. It is described in detail in Chapter III. 
CHAPTER III

PRESENTATION OF DYNAMIC MODEL

The proposed departure dynamic model incorporates a computational aerodynamics package for the definition of the forces and moments which are input to the rigid-body equations of motion. This chapter presents a complete development of the model. The nonlinear lifting line procedure is implemented by extending, as much as possible, classical lifting theory to individual wing vortex panels. A local wing coordinate system is introduced to facilitate the calculation of each station's flow properties. Digital integration of the rigid-body equations necessitates the use of a body-fixed axis system for the representation of state variables, forces and moments. Finally, a coordinate system fixed in the aircraft's physical trajectory space is required to keep track of the vortex lattice wakes shed from the translating aircraft. The model thus entails mathematical operations in three coordinate systems and the transfer of information between them. Presentation of the dynamic model begins with a discussion of these right-handed axis systems.

\section{III.1 Coordinate Systems}

The aircraft geometry as represented by the placement of bound vortex segments and corresponding control points on all lifting surfaces is given in Figure 1. This geometric configuration is typical of general aviation aircraft. Although these light, single-engine airplanes are the main focus of the present development, it is pointed out that the 
dynamic model with the possible addition of fuselage effects is applicable to all types of aircraft (excluding high leading-edge sweep examples). A body-axis system is set up in the vehicle with the origin coincident with the center of gravity; the $x$-axis points down the fuselage center line, positive toward the nose, $y$-axis positive out the right wing and z-axis completing the right-hand triad. Each vortex segments is considered to be a vector whose direction is indicated by the sense of its circulation in a right-handed rule fashion. The location of the head (and tail) of each vector is described by a three-dimensional address $(x, y, z)$ with respect to the body-axes origin. The spacial orientation of each control point is similarly represented. A specific vehicle geometry may thus be constructed based on the longitudinal and vertical positions of the lifting surfaces relative to the origin and their orientations relative to the body axes. In particular, the main wing center-span quarter-chord point is located a given distance forward of the origin; the relative positions of its left and right semispan panels are functions of sweepback, dihedral and pitch incidence angles. Similar construction is used in positioning the horizontal tail except that additional provision for vertical placement above the main wing plane is made. Vertical fin alignment is accomplished by considering it to be a swept "wing" at $-90^{\circ}$ dihedral and zero pitch incident; the tail of its first (inboard) vortex vector is longitudinally and vertically prescribed rather than the fin center-span point.

Once this "catalog" of vortex elements and control points is formed, it is preserved in the dynamic model as a rigid-body airframe geometry. 
This aircraft model can now be "flown" through inertial space by integrating the equations of motion. Appendix A presents these equations formulated in terms of six state variables: axial, normal and lateral velocity components and pitch, roll and yaw angular rates. Expressions governing the time variations of pitch, bank and heading angles relative to an inertial-axis system are also given. Thus, geometry definition and motion simulation require the specification of a body-fixed axis system (Fig. 2).

If the individual vortex segments are assumed to act like 20 airfoils at local effective angles of attack, it is then necessary to define local-wing coordinate systems in order to compute these angles. The origins of the local-axis systems are the midpoints of the bound elements while their orientation with respect to the body axes may be derived by a sequence of Euler angle rotations involving the dihedral, sweep and pitch incidence of the associated lifting surface. The local $x$-axis lies in the plane formed by the bound vortex and the control point, positive toward the nose, the $y$-axis lies along the lifting line, positive toward the right and the z-axis completes the triad. The matrix transformation from the body-axis system to a local-wing system is given in Appendix $B$. The normal velocity to each bound element is another quantity appearing in the lifting line theory which is readily calculated in the local-wing space. Finally, on completion of the elemental force computations, the direction of the resultant is resolved locally and then transformed into body-axis components for use in the equations of motion. The local computations described above are formulated in Appendix $C$ for a typical spanwise station. 
Analogous to the vector representation of the vehicle geometry in the body-axis system is the spacial arrangement of shed vortex segments in an inertial system. At any particular time during the flight simulation, the geometric relationships between the control points fixed on the aircraft and the wake lattice elements are known from their inertial space addresses. The transformation matrix from the body-axis system to the inertial-axis system given in Appendix $B$ is used to orient the geometric model in inertial space according to current pitch, bank and yaw attitudes. This transformation is also useful in computing the instantaneous aircraft position relative to the inertial origin (coincident with the body-axes origin at trajectory initialization). The aircraft's center of gravity position is found by transforming the body velocity components known from the equations of motion into inertial components and integrating. Precise representation (both location and attitude) of the airplane model in the inertial coordinate system is essential to aircraft/wake positioning for the downwash calculations. An example of the downwash contribution of an arbitrary wake element at a particular wing control point is presented in Appendix $D$.

\section{III.2 Vortex System}

The vortex system used in the analysis is pictured in Figure 3 in its initialized form. The system is three-dimensional consisting of the superposition of three wakes trailing from the main wing, horizontal tail and vertical fin. Initially, the wakes are assumed to lie in the plane created by their associated bound vortices and the inertial $x$-axis. The wing, tail and fin lifting lines are segmented into equal-span 
elements. Results are obtained in this paper for eight element wing and tail lifting lines and a three element fin. Trailing aft of each bound segment is a parallelogram lattice element formed by two streamwise vortex legs closed by a shed transverse vortex at a downstream distance of one (main wing) chord. This lattice arrangement is repeated until the system has a streamwise dimension of about two fuselage lengths. Assigned to each parallelogram element is a circulation strength $\Gamma_{j, k}$ where $j$ denotes a particular spanwise station and $k$ signals the streamwise position. Figure 4 shows the sense of circulation associated with a typical lattice element and its geometric relationship to a given control point. Initially, the circulation strengths of streamwise lattice parallelograms are set equal to their corresponding $\Gamma_{i, 1}$ value resulting in a system whose transverse vorticity vanishes due to the opposite sense of neighboring elements.

A series of calculations were performed to determine the streamwise number of elements which must be retained in the span load computations. Single wing improvement in total load estimation by using five streamwise lattice parallelograms was less than $1 \%$ over that calculated with four; therefore, each surface load distribution is assumed to be adequately computed by using four streamwise elements of its own wake. Further tests showed that the empennage lifting lines and trailing wakes had negligible effect on the main wing calculations. On the other hand, the wing bound and free trailing vorticity was very significant in the tail load estimations. The results lead to a wake model consisting of seven streamwise wing lattice elements, four of which are used in wing load 
calculations, and four streamwise parallelograms for each of the tail and fin surfaces. The tail load calculations are performed with the complete seven-four-four wake configuration. The seven chord-length dimension is about two fuselage lengths for the present aircraft geometry.

As the simulation commences and the rigid airframe translates away from its initial vortex wake, the system geometry and circulation strength assignment must be updated. For example, as the airplane advances during the first integration step, the new location and orientation of the three surface lifting lines redefine the first streamwise row of parallelgram elements. These elements are formed by the bound vortex segments and their trailing vortex legs extending rearward to the transverse vortices deposited in the previous position of the airframe. The strengths of these redefined vortex units are fixed by the nonlinear lifting line procedure. The other elements take on the spacial positions and strengths identified with their immediate forward streamwise parallelograms in the previous time step. This updating sequence never requires recording more than the seven-four-four configuration outlined above. Of course, the regularity of the initial vortex system is disrupted with commencement of the "flight" and may become extremely nonplanar in highly dynamic portions of the trajectory. Sti11, an approximate mean chord-1ength element dimension can be maintained throughout the simulation by selecting an appropriate time step with respect to forward velocity. (The discussions presented in the remainder of this chapter refer specifically to the main wing for generality of explanation although direct analogs exist for the empennage.). 


\section{III.3 Nonlinear Lifting Line Procedure (Levinsky)}

The lifting line assumption states that each spanwise panel of the wing acts like a two-dimensional airfoil at an effective angle of attack equal to the difference between the local pitch angle of attack and the downwash angle of attack induced by the trailing vortex systems. For a wing station $i$, the lifting line equation is

$$
\alpha_{e f f_{i}}(t)=\alpha_{p_{j}}(t)-\alpha_{d_{j}}(t)
$$

This theory assumes that the two-dimensional, steady state, nonlinear functional relationship between lift and angle of attack for a given airfoil geometry can be imparted to the individual panels:

$$
C_{L_{i}}(t)=C_{L_{i}}\left\{\alpha_{e f f i}(t)\right\}
$$

Implicit in this assumption is the requirement that the chordwise pressure distribution, which affects the nature of flow separation on the airfoil, achieves the steady state distribution for the current angle of attack on a shorter time scale than that associated with wake and vehicle dynamics (Ref. 13). Since the section lift curve is independent of time, the unsteady effects must enter through the time dependence of $\alpha_{\text {eff }}$. As Equation (1) states, this may be introduced through the local pitch angle of attack which is a function of the vehicle's dynamics and/or the induced angle of attack which depends on the vortex system's circulation strengths. The calculation of the pitch angle of attack at a local station $i$ is presented in Appendix $C$. 
The control point for evaluating $\alpha_{\text {eff }} f_{j}$ is located a distance $x_{C p_{i}}$ from the leading edge on a line parallel to the body system $x$-axis and intersecting the midpoint of the local bound vortex segment. As indicated in the Introduction, the present dynamic model uses the standard Prandt l lifting line technique of placing the bound vortex segment at the local $c_{j} / 4$ distance aft of the leading edge and the associated control point at $x_{C p_{i}}=3 / 4 c_{i}$. Hence, there is no induced velocity contribution from the wing bound vortex segments. However, in order to generalize the model for arbitrary $x_{C p_{j}}$, the following formulation due to Levinsky (Ref. 13) is included: let the downwash angle $\alpha_{d_{j}}$ be equal to the three-dimensional downwash angle $\alpha_{3 D_{j}}$ due to the complete vortex system (bound and free trailing vorticity) at the control point less an equivalent two-dimensional downwash angle $\alpha_{2 D_{i}}$ induced by an infinite span bound vortex $\left(\Gamma_{i, 1}\right)$ at $c_{i} / 4$.

$$
\alpha_{d_{i}}(t)=\alpha_{3 D_{i}}(t)-\alpha_{2 D i}(t)
$$

Let the downwash component induced by the $j^{\text {th }}$ spanwise, $k^{\text {th }}$ streamwise parallelogram lattice element (of unit circulation) at control point $i$ be denoted $\Delta_{4} w_{i_{j, k}}$. Then,

$$
\alpha_{3 D i}(t)=\arctan \left\{\frac{1}{u_{1}} \sum_{j=1, k=1}^{j=N, k=M} \Delta_{4} w_{i_{j, k}} \Gamma_{j, k}(t)\right\}
$$

Here, the summation is over all $N$ spanwise by $M$ streamwise lattice elements. The local axial velocity component $u_{1}$, arises from the standard definition of angle of attack as the inverse tangent of the 
ratio of normal to axial velocity components. (See Appendix C.) Mathematical expressions for the downwash components $\Delta w_{i_{j, k}}$ (there are four composing each parallelogram element $\left.\Delta_{4} w_{i_{j, k}}\right)$ are derived in Appendix $D$ in terms of vector representations of the wake vortex segments and control points. The equivalent two-dimensional downwash angle is

$$
\alpha_{2 D_{j}}(t)=\arctan \left\{\frac{1}{u_{1}} \frac{\Gamma_{i, 1}(t)}{2 \pi\left(x_{c p}-x_{c / 4}\right)_{i}}\right\}
$$

Finally, the strengths of the individual bound vortex segments are fixed by imposing the Kutta-Joukowski Law

$$
L_{i}(t)=\rho V_{N_{j}} \Gamma_{i, 1}(t)
$$

Expressing the lift in two-dimensional coefficient form and solving for the bound circulation strengths yields

$$
\Gamma_{i, 1}(t)=\frac{1}{2} V_{N_{i}} C_{i} C_{L_{i}}\left\{\alpha_{e f f_{j}}(t)\right\}
$$

where $V_{N_{i}}$ is the local velocity component normal to the bound vortex evaluated at the segments' midpoints. (See Appendix C.)

A derivation for the generalized form of the two-dimensional KuttaJoukowski Law applicable to the unsteady case, Eq.(6), is presented in Reference 13. The formulation begins by applying the relationship equating lift magnitude to the negative rate of change of total momentum (associated with transverse vortex segments) to a discrete vortex wake system. The conditions that the shed vortex elements are convected downstream at velocity $V_{N_{j}}$ and that the total vorticity must remain zero for all time are used in developing momentum expressions for two consecutive time steps. In the limit as the step size tends to zero, the finite difference form of 
the momentum terms yields the generalized Kutta-Joukowski Law. This form is considered adequate for modeling wing-span load distributions in the present application. Reference 13 examines the lifting line equations (1) and (7) for their compatibility with existing aerodynamic load prediction methods. For example, in the case of a linear lift curve with steady state conditions, the lifting line equations ( $N$ spanwise elements) reduces to the usual form of a single matrix equation which may be solved explicitly for the circulation strengths. Additionally, if the control points are placed at the $75 \%$ chord location and a linear lift curve is used, the lifting line formulation reduces to the Weissinger L-method in the steady case. Reduction to the unsteady form of the Weissinger theory for both two-dimensional and three-dimensional flows is also verified. Finally, close correspondence between the discrete vortex formulation and the continuous vortex sheet theory of Wagner in calculating lift due to a step change in angle of attack establishes the validity of using a one-chord-length distance in the shedding of transverse vortices (Ref. 13).

\section{III.4 Aerodynamics/Simulation Integration}

The lifting line expressions given by Equations (1) through (7) can be formulated as a system of $2 \mathrm{~N}$ algebraic equations in the $2 \mathrm{~N}$ unknown quantities $\Gamma_{i, 1}(t)$ and $\alpha_{\text {eff }}(t)$ where $i=1,2, \ldots, N$. This system must be solved for each step in time during the flight trajectory. Since the equations include the nonlinear stall and poststall effects from the 2D lift curve, an iteration procedure is employed for their solution. As shown in the flowchart of Figure 5 , this iteration scheme appears as a computational loop interior to the flight simulation 10op. Given an 
aircraft geometry with segmented lifting surfaces characterized by $2 D$ profile lift curves, the vehicle dynamic parameters and the vortex wake geometry are known at a specific time interval denoted by the index $j$. The instantaneous pitch angle-of-attack distribution $\alpha_{p_{j}}{ }^{j}$ is determined from the expressions developed in Appendix C. In order to evaluate the lifting line effective angle-of-attack assumption, a spanwise distribution of induced angles must be specified. Initially, this may be accomplished by inputting a guessed distribution, or, after at least one integration step, the assumed spanwise variation for the current time interval $j$ may be set equal to the converged solution of the previous step. The effective angle of attack defined by Eq.(1) appears in Figure 5 with superscripts $j, m$ denoting time step and iteration step, respectively. The bound circulation strengths are adjusted according to the KuttaJoukowski Law, Eq.(7). Now the actual induced distribution $\tilde{\alpha}_{d_{j}}{ }^{j, m}$ is calculated from the downwash effects of the bound and free vorticity. (See Appendix D.) Levinsky gives the induced angle of attack update equation: $\quad \alpha_{d_{j}}^{j, m+1}=\alpha_{d_{j}}^{j, m}+C\left(\tilde{\alpha}_{d_{j}}^{j, m}-\alpha_{d_{j}}^{j, m}\right)$

Equation ( 8 ) says the current $m^{\text {th }}$ iterative value is added to the difference between it and its associated wake-derived value modulated by the weighting factor $C$. The procedure is repeated (the $m$ loop in Fig. 5) until convergence is obtained. In the present application, convergence requires that two successive estimations may not differ by more than $0.0057^{\circ}$ for all main wing elements and $0.57^{\circ}$ for all tail and fin stations. Upon convergence, the body forces and moments are computed from the 1 ifting surfaces' loading based on the $\alpha_{\text {eff }} \boldsymbol{j}$ distribution (Appendix $c$ ) and 
one integration step is performed (the $j$ loop in Fig. 5). The resulting rotation and translation of the airframe model redefines the vehicle dynamic parameters and the vortex system geometry whence the iteration loop can be re-entered.

Several notes are in order before concluding this presentation of the dynamic departure model. The capability of "biasing" the search for a converged induced angle-of-attack solution is realized through the initial guess option in the iteration procedure (Fig. 5). At any point during the flight trajectory, the procedure may be "directed" to search in a particular region where solutions are expected or desired. For instance, as discussed in Chapters IV and $V$, the introduction of an asymmetric induced angle-of-attack distribution during stall penetration leads to the identification of multiple lifting line solutions. The multiple solution phenomenon has been verified to be characteristic of lift discontinuities at the stall. Asymmetric induced angle guesses applied at low angles of attack result in converged symmetric solutions. Thus, load distribution uniqueness expected for conventional flight conditions is exhibited by the model.

Experience has shown that the iteration technique disallows individual panel solutions on steep negative slope regions of the section lift curves. Therefore, a modified version of the special stalled-element logic proposed by Levinsky was incorporated into the present model. When

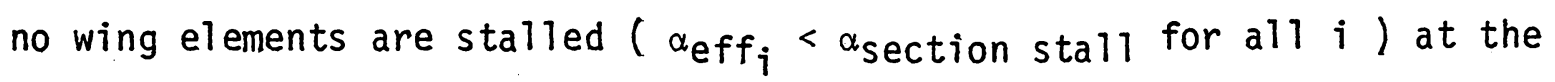
prior time step, the current step initial $\alpha_{d_{j}} j, 1$ distribution is taken equal to the previous converged solution or guessed as mentioned above. 
If one or more wing elements stall during the current iteration sequence, then the procedure is restarted assuming that the induced angles of those stalled elements are such that their corresponding effective angles of attack are in the fully stalled region of the lift curve. This logic forces any stalled elements to remain stalled as long as the lifting line equations permit. The lift hysteresis loop and the pitch angle-of-attack range under which multiple solutions exist are maximized. The stalledelement method also yields results indicating the presence of multiple stall cells discussed in Chapter IV.

Finally, the speed at which convergence is achieved is regulated by the weighting factor $C$ (see Eq. 8). Increasing $C$ speeds up the convergence, but too large values can destabilize the iteration procedure. The choice for $C$ values depends on wing configuration, the number of spanwise elements and lift curve shape. For the present model the maximum stable $C$ value for the wing, which is analyzed independently of the empennage, was determined to be 0.4 . The horizontal tail and vertical fin calculations use different $C$ values, 0.15 and 0.075 respectively, al- . though their solutions are converged simultaneously based on the complete aircraft wake as discussed in section III.2. The iteration procedure becomes unstable for any $C$ value when either of the free trailing vortex legs of a bound segment closely approach the associated control point. This problem was solved by ignoring the downwash contribution of any vortex element within a radius of $8 \%$ chord (main wing) from any control point. 


\section{CHAPTER IV}

\section{PRESENTATION OF RESULTS}

The stall departure dynamic model presented in Chapter III and detailed in Appendices $A-D$ was analytically formulated and developed into a FORTRAN computer program. The code was written to maintain complete flexibility as pertains to aircraft geometry definition and initial flight conditions. However, the nature of the nonlinear lifting line aerodynamics generator precludes the investigation of certain flight applications, for example, high speed flight or large leading-edge sweep configurations. Light, single-engine general aviation airplanes are probably most suited for analysis with the present dynamic model. Results of computations performed for the Grumman American AA-1 Yankee airplane are presented in this paper. The Yankee has a low, straight, untapered main wing and tail surfaces of moderate sweep angles and taper ratios. Simple geometry (Fig. 1), a large experimental data base and NASA documented full scale flight experience make this configuration a prime test candidate.

\section{IV.1 Lift Curve Characteristics}

The configuration data necessary in constructing the mathematical airframe geometry and the mass properties imparted to the vehicle for flight simulation are listed in Table I. These data reflect the full scale values of the test aircraft described in Reference 2 . The $2 D$ characteristic lift curves for the wing profile section NACA $64_{2}-415$ and the tail surfaces NACA $65_{1}-012$ are presented in Figures 6 and 7 . 
The data were obtained from Reference 29 with the following necessary modifications. The section lift coefficient data for the NACA $64_{2}-415$ airfoil is given only up to $20^{\circ}$ angle of attack. The fully stalled lift characteristics are estimated by assuming the difference between the maximum lift coefficient and the minimum poststall value is the same for the $2 D$ section as for a finite aspect ratio $A R=7$ wing of the same profile (Ref.5). Similarly, the poststall positive lift curve slopes of the two wings are equated. This results in a poststall slope equal to $1 / 9$ the value of the prestall lift curve slope and is comparable to section 1 ift characteristics used by Levinsky (Ref. 13) in testing his nonlinear lifting line theory. The data for the NACA $65_{1}-012$ profile, used for both the horizontal tail and vertical fin, are available through an angle of attack of $16^{\circ}$ (Ref. 29). The corresponding lift coefficient is assumed to be the poststall minimum and a positive poststall 1 ift curve slope equal to $1 / 9$ of the prestall value is applied. The 1 ift properties of this symmetric airfoil are extended into the negative angle-of-attack range by reflecting the curve about the $\alpha=0^{0}$ and $C_{L}=0$ axes.

One of the goals of the present formulation is the capability of analytically assessing the departure resistance benefits achieved by configuration modifications. An example is the application of drooped leading edges on the outboard wing panels mentioned in the Introduction. Such a modification is easily incorporated into the present model by imparting drooped leading edge lift characteristics to the outboard vortex segments. Because no 20 drooped leading edge $64_{2}-415$ data were available, the following procedure was used to estimate these lift 
characteristics: Beginning with the 20 unmodified NACA $64_{2} 415$ section data of Fig. 6, graphically add the 7 ift curve of the full scale Yankee aircraft with full span leading edge droop given in Reference 30 . Now, remove fuselage, three-dimensional and $64_{2}-415$ section effects by graphically subtracting the lift curve of the full scale Yankee-withbasic-wing (Ref. 30). Any discontinuities in the resulting graph are smoothed. The estimated lift characteristics of the drooped 642-415 modified airfoil section are given in Figure 8 . Comparison of the droop model with the basic section data shows an increase in maximum 1 ift coefficient, 1.75 from the original 1.49, and a delay in occurrence of $C_{L_{\text {MAX }}}$ from $14.6^{\circ}$ angle of attack to $22.5^{\circ}$. This estimated model is assumed a reasonable representation of drooped leading edge lift charateristics at least up to $C_{L_{M A X}}$. For the results presented in Chapter $V$, the local angle of attack on the drooped sections did not greatly exceed alpha for $C_{L_{\text {MAX }}}$.

One final aerodynamic consideration concerns the modeling of fuselage effects. Although no body influence is included in the lifting line calculations, an attempt is made to introduce fuselage axial forces into the equations of motion by using static wind tunnel data. The dependence of axial force coefficient for the Yankee aircraft on angle of attack is given by experimental data in Reference 31 . These data are analytically expressed by the two equations

$$
\begin{array}{ll}
C_{X}=3.5787 \alpha^{3}+2.1810 \alpha^{2}+0.2183 \alpha-0.0238 & \text { for } \alpha \leq 13.82^{\circ} \\
C_{X}=-1.0154 \alpha^{3}+2.9862 \alpha^{2}-2.1668 \alpha+0.6905 & \text { for } \alpha>13.82^{\circ}
\end{array}
$$


The versatility of the dynamic model is exampled by its usefulness in performing several kinds of flight analysis; the emphasis of course is on stall and poststall applications although low angle-of-attack flight may also be simulated. Two specific capabilities of the model are illustrated and discussed in Chapter V: (1) Analytical generation of forced-roll oscillation data. In this technique the dynamic stability parameter $C_{L_{p}}+C_{L_{\dot{\beta}}} \sin \alpha$ is determined by rolling the "aircraft" according to a prescribed forcing function. (2) Six degree-of-freedom flight simulations. Neither the aerodynamics nor the vehicle dynamics are known apriori, but they are computed interactively to produce the flight trajectory. The only prescribed parameter other than the initial flight conditions is the elevator control sequence. The methods by which these analyses are accomplished are outlined below.

\section{IV.2 Analytical Forced-Roll Oscillation Data}

The computational production of forced oscillation data results from an attempt to mathematically simulate the wind tunnel procedure developed by NASA to measure dynamic stability parameters. Specifically, forced-roll oscillation tests are used to define the aerodynamic damping in roll of a given configuration. A scale model is mounted to a pivoted sting assembly with an internal strain gauge. The model-sting combination is forced to oscillate in roll by an electric motor through a flywheel and bell-crank assembly. These forced oscillations are performed at various angles of attack. Outputs from the balance are analyzed to separate the signal into components in phase with, and completely out 
of phase with, the angular displacement of the model. The out-of-phase components are then used to compute the damping-in-roll stability derivatives (Ref. 2). The mathematical expressions for reducing the data presented in Appendix $E$ are due to Reference 32.

This procedure can be analytically reproduced with the present formulation by prescribing an angle of attack (pitch attitude) for the geometric model and forcing it to roll about its $x$-axis according to an input function as it translates forward. The output signal mentioned above is the time dependent roll moment. The calculations of Appendix $E$ are performed digitally. Figure 9 presents the output signal, roll moment coefficient due to wing only, for the input function

$$
\phi=15^{\circ} \sin .6 \pi t
$$

at a pitch attitude of $5^{\circ}$. This function duplicates the oscillation amplitude and frequency used in obtaining the wind tunnel measurements against which the computed data are compared. The forward velocity is set at $64.7 \mathrm{ft} / \mathrm{sec}$ to maintain roughly a one-chord length streamwise separation between shed transverse vortices during a 0.068 second interval. This interval is used since one complete oscillation cycle ( 3.33 seconds) can be accomplished in exactly 49 steps. Figure 10 gives the forced-roll output for the full configuration (wing and empennage) at $\theta=14^{\circ}$ while the output data (wing only) for the poststall pitch attitude of $\theta=18^{\circ}$ is shown in Figure 11. Comparisons of the angle-of-attack variation of the computed parameter $C_{L_{p}}+C_{L_{\dot{\beta}}} \sin \alpha$ and wind tunnel measurements are made graphically in Figure 12. Both 
the basic wing and the outboard drooped leading edge modified wing computations are presented. The drooped outboard data were generated analogous to the basic wing except that the two outboard vortex elements on either semispan were assigned the lift characteristics of Figure 8.

\section{IV.3 Six Degree-of-Freedom Flight Simulations}

The major objective of this research is the analytical simulation of poststall departure flight motions. Four trajectories in which the aircraft is "flown" through the stall are presented to aid in describing the mechanism of the departure and factors which influence its development. The success achieved in simulating the abrupt wing drop departures attests to the integrity of the vehicle dynamics/aerodynamics modeling concept.

All trajectories are flown from an initial trimmed flight condition with no thrust, simulating an engine off or idling condition. A computer routine was written and used in conjunction with the dynamic model to trim the aircraft at a given pitch attitude, say $10^{\circ}$. The routine adjusts the pitch incidence of the horizontal tail relative to the body axis system (angle $v$ in Appendix B) until the pitch moment due to the wing is equilibrated by that due to the tail. For the $10^{\circ}$ initial pitch attitude, this "elevator" trim angle is $4.56^{\circ}$ leading edge down. Since the tail incidence angle may be continually changed throughout the trajectory according to a prescribed logic, the movable tail acts as a stabilator. This control surface is used to move the aircraft from its initial $10^{\circ}$ angle of attack to the stall angle of attack, about $14^{\circ}$, by 
using a step command from the trim angle to an arbitrarily chosen $-9^{\circ}$. As the aircraft penetrates the stall, the stabilator is stepped again to its assumed maximum value $-15^{\circ}$. This control sequence approximates a pitch-up through the stall followed by an abrupt "stick full-back" maneuver at the stall break. The initial conditions of all trajectories are a $10^{\circ}$ pitch attitude, wings level trimmed aircraft with velocity of $103 \mathrm{ft} / \mathrm{sec}$ (slightly above the $1 \mathrm{~g}$ stall speed) and altitude of $3000 \mathrm{ft}$. The trajectories are presented as investigations of three poststall phenomena: (1) the existence of multiple lifting line solutions, (2) the effect of flight asymmetries during stall penetration, and (3) the effect of configuration modifications on departure. Figure 14 presents the time histories of the longitudinal variables of the Yankee aircraft as it penetrates the stall. There are no initial flight asymmetries and the induced angle-of-attack distribution required at the beginning of each iteration procedure is taken to be the last converged solution. The total elapsed time of flight is 5.0 seconds and the integration time step is 0.04 second. The lateral components are flat zeros throughout the simulation and are not presented. An identical trajectory is described by Figure 15 except that beginning with the stall penetration, an asymmetric induced angle distribution was input every time step for an arbitrary 30 intervals. Both longitudinal (Fig. 15.a) and lateral variables (Fig. 15.b) are plotted for this wing drop departure represented in physical space in Figure 15.c. The influence of asymmetric flight conditions is examined in Figure 16 where an initial sideslip of $-5^{\circ}$ is prescribed. The figure presents the time trace of all flight parameters. 
In each of the above simulations, the vehicle is configured with the basic NACA $64_{2}-415$ profile section. The final trajectory, Figure 17, repeats the scenario of Figure 15 for an aircraft with the two outboard vortex elements on either semispan assigned the $1 \mathrm{ift}$ characteristics of the drooped leading edge airfoil.

Before proceeding with a detailed discussion of the dynamic simulations in Chapter $V$, it is encouraging to note that the stall patterns calculated for steady flow conditions by this model are observable in a physical situation. Consider the oil flow visualization photograph of Figure 13 which shows the existence of two stall cells on an aspect ratio $A R=6$ wing (Ref. 33 ). The flow is attached from the leading edge to the trailing edge near both wing tips and appears attached over most of the midspan chord. This pattern is repeated by the computational model on an $A R=6$ wing; a stalled element is represented in Figure 13 by a local reduction of lift in the spanwise distribution. The outer two panels on either wing tip and the inner two panels at the center span are unstalled while the two symmetrically stalled panels resemble stall cells. The stalled panels, due to their reduced circulation strengths, allow adjacent panels to experience large induced angles of attack. This yields prestall effective angles of attack for the adjacent panels and produces the stall-cell configuration. A more realistic representation of the physical stall cells is likely through a finer discretization of the lifting line wing model. 
CHAPTER V

\section{DISCUSSION OF RESULTS}

\section{V.1 Forced Oscillation Data}

Reference 3 examines the importance of mathematically defining the dynamic stability derivatives in performing high angle-of-attack flight analysis. Indeed, these parameters are required in stability computation and mode identification throughout an aircraft's entire flight envelope. Information concerning the aircraft's damping in roll, yaw and pitch characteristics is provided through the derivatives $C_{\ell_{p}}+C_{\ell_{\dot{\beta}}} \sin \alpha, C_{N_{r}}+C_{N \dot{\beta}} \cos \alpha$ and $C_{M_{q}}+C_{M_{\dot{\alpha}}}$, respectively. The major indication of lateral instability at the stall resulting in possible wing rock or wing drop is the quantity $C_{\ell_{p}}+C_{\ell_{\dot{\beta}}} \sin \alpha$. The determination of this term by forced-roll oscillation at a particular angle of attack is accomplished through the method of Appendix $E$. A plot of $C_{\ell_{p}}+C_{\ell_{\dot{\beta}}}$ sina as a function of angle of attack is presented in Figure 12.

The most important characteristic of the damping-in-roll derivative is the change in sign which indicates a shift in lateral stability. The reason for sign crossover can be explained by examining the output signal traces for prestall and poststall angles of attack given in Figures 9-11. The roll moment due to the wing alone (output signal of Appendix E) at the $5^{\circ}$ pitch attitude of Figure 9 appears as a $90^{\circ}$ out-of-phase cosine curve with respect to the bank attitude sine curve (input function). No wing elements stall during the complete cycle. The maximum positive 
roll moment occurs at half cycle when the wings are level but the roll rate (derivative of bank attitude) is at its maximum negative value. The left semispan elements experience an increase in angle of attack over the aircraft's pitch attitude due to a py/u term while the righthand vortex segments experience a corresponding decrease. Since there is no stalling, the left wing has more lift than the right wing yielding a positive roll moment. The same argument can be made for the maximum negative roll moment values. Zero roll moments occur during the transition from positive to negative (and vice versa) roll rates. The integration of the output function multiplied by the out-of-phase component of the input function yields a negative $C_{\ell_{p}}+C_{\ell_{\dot{\beta}}} \sin \alpha$ term.

At the poststall pitch attitude of $18^{\circ}$ (Fig. 11), all elements except the wing tips would be stalled if the aircraft had no roll motion. At the half cycle point (maximum negative roll rate), the py/u contribution stalls all left wing elements and unstalls the three outboard panels on the right semispan. The major portion of the right wing is operating at high prestall angles of attack and thus produces greater lift than the stalled left wing. The result is that a maximum negative roll moment occurs at half cycle, opposite the case of Figure 9. Similar counter arguments apply for the maximum positive roll moment values and the result is the appearance of an output curve shifted by one-half cycle relative to Figure 9. This shift accounts for the positive $C_{\ell_{p}}+C_{\ell_{\dot{\beta}}} \sin \alpha$ calculation; the difference in sign of the roll damping term between the prestall $\alpha=5^{\circ}$ and poststall $\alpha=18^{\circ}$ cases 
originates in the cycle integrations (Appendix $E$ ) of the functional form $\cos (x(t)) f(t)$ compared to the term $\cos (\pi-x(t)) f(t)$.

Figure 10 presents the roll moment time trace due to the wing and empennage at $14^{\circ}$ pitch angle for a complete roll oscillation cycle. The jumps in the curve are due to the stalling of individual elements. There is no apparent sinusoidal form of the output curve as in the previous examples. However, the curve does exhibit rather frequent, constant amplitude fluctuations about zero roll moment which, when digitally integrated against the out-of-phase component of the bank angle function, result in a near zero $C_{\ell_{p}}+C_{\ell_{\dot{\beta}}} \sin \alpha$ value. This is the crossover point ( $\alpha=14^{\circ}$ for this aircraft) indicative of lateral stability shift. The difference between the wing alone and full configuration calculations is negligible even at this critical point (Fig. 12.a). In addition to the examples discussed above, several more wing alone $C_{\ell_{p}}+C_{\ell_{\dot{\beta}}}$ sin $\alpha$ estimations were made at various pitch attitudes; the resulting variation of the damping-in-roll term with angle of attack is presented in Figure 12.a along with wind tunnel data for comparison.

The data of Figure 12.a for the unmodified wing shows that the present analytical technique predicts the sign change in $C_{\ell_{p}}+C_{\ell_{\dot{\beta}}} \sin \alpha$ very well in accordance with the wind tunnel data (Ref. 2). Although the correlation breaks down away from the crossover point, the trends of the experimental data are well exhibited. In fact, the computed curve has a constant vertical shift downward relative to the test data. In light of the modified wing results presented in Figure 12.b, this 
shift appears to have no special significance. Several factors are suggested for the difference between the analytical results and test values: (1) It is likely that the wind tunnel model and the analytical model were roll oscillated about different body $x$-axes. The physical model's x-axis may have been an arbitrarily defined model axis convenient for mounting the aircraft on the oscillation rig. The analytical method forces roll motion about the $x$-body axis representing the fuselage centerline to which the main wing and empennage are assumed attached. (2) A finer discretization of the wing lifting line and the trailing vortex wake may more accurately represent the dynamic lift distribution. (3) Fuselage effects which are not analytically modeled influence the inboard wing flow field and become increasingly important with higher angles of attack. (4) The basic input to the mathematical model is the sectional lift curve and, as described in Chapter IV, the poststall $C_{L}-\alpha$ characteristics had to be estimated. Despite these potential sources for data disagreement and in view of the fact that no fine tuning of the system was attempted, the analytical technique is considered to be an excellent dynamic stability parameter estimator. Forced-roll oscillation analys is performed for the outboard leading edge droop modification is presented in Figure 12.b. The trends of the wind tunnel test data (Ref. 2) again are well predicted al though the droop leading edge was only applied to the outer $38 \%$ of the experimental model's semispan while $50 \%$ was used on the analytical model (for convenience). Neither model shows a change in sign for the roll damping term 
up through $20^{\circ}$ angle of attack. The decrease in the roll damping at about $14^{\circ}$ is due to the stalling of the inboard unmodified section of the segmented wing. The computational results verify that the effective angles of attack on the drooped sections do not greatly exceed the $\alpha$ for $C_{L_{\text {MAX }}}$ during the aircraft pitch sweep up to $20^{\circ}$. Hence the outboard portions of the wing, which have the major influence in roll stability, are operating unstalled similar to the $\alpha=5^{\circ}$ basic wing case. The wing alone and full configuration calculations again show the negligible influence of the tail assembly loads. The computation values of $C_{\ell_{p}}+C_{\ell_{\dot{\beta}}} \operatorname{sin\alpha }$ may diverge from the experimental curve at higher angles of attack since the estimated $2 \mathrm{D}$ drooped leading edge 1 ift curve is questionable at poststall angles. These modified wing calculations are of course subject to the same experimental/analytical discrepancy factors listed for the basic wing configuration; still, the beneficial stability effects of the droop outboard leading edges are well represented.

The ability of the proposed dynamic model to predict the major characteristics of the angle-of-attack variation of the damping-in-roll parameter $C_{\ell_{p}}+C_{\ell_{\beta}} \sin \alpha$ may prove extremely valuable in the design phase flight analysis of future configurations. The success of the forced-roll calculations also lends credence to the utility of the model in simulating poststall flight departures. The results of several simulations are discussed in the following section.

\section{V.2 Stall Departure Flight Simulations}

The flight trajectory represented in Figure 14 by time histories 
of the vehicle's longitudinal variables serves as a basis for comparisons of the wing drop departures. The aircraft configuration is modeled as shown in Figure 1 and specified in Table I with the basic unmodified NACA $64_{2}-415$ airfoil section. The initial flight conditions represent a laterally trimmed aircraft. The elevator control sequence given in Figure 14 is as described in Chapter IV. No asymmetric induced angleof-attack solution searches are implemented during the trajectory.

The pitch attitude, angle of attack and pitch rate time traces all indicate a short period oscillation of constant cycle duration 1.4 seconds. This is due to repeated stall penetration resulting from applying full elevator. As the aircraft pitches through the stall $14^{\circ}$ to about $23^{\circ}$ angle of attack (initial penetration), all wing vortex segments stall symmetrically. The loss of lift causes a pitch down moment created by a large horizontal tail contribution and subsequent reduction to prestall angles of attack. As lift is regained, the positive moment forces another stall penetration resulting in the oscillatory motion displayed in Figure 14. Throughout the trajectory, the pitch attitude oscillates about a decreasing mean curve while the angle of attack oscillates about a poststall value of $18.5^{\circ}$. The decay of pitch rate oscillation shows that this stalled, low pitch attitude flight condition is stable. The total velocity time history shows a decrease as the aircraft penetrates the stall followed by an increase over the last half of the flight. This is evidence of the initiation of a moderate sink rate; the resolution of the velocity into horizontal and vertical inertial-axis components verifies the build-up of sink rate 
while forward translation remains fairly constant. Due to the repeated symmetric stall/unstall cycle there is no roll-off tendency; the lateral variables (not shown) are zero throughout the simulation. The term usually applied to the "staggering motion of an aircraft" described above is "mush" (Ref. 12), where the vehicle angle of attack is such that partial stalling of the wing occurs. This motion has not been recorded in flight tests for the Yankee configuration modeled here suggesting that another poststall flight solution exists. However, it is noted that general aviation airplanes of slightly different configuration do exhibit this symmetric stall characteristic.

The search for alternative solutions to the symmetric stall penetration case was initiated by taking advantage of the multiple solution phenomena associated with discontinuous lift curves. Figure 15 presents the results of a trajectory with identical flight conditions and elevator control sequence as Figure 14. However, beginning with stall penetration and continuing for 30 time intervals, the computation model was "directed" to search for assymmetric lifting line solutions by supplying the iteration procedure with an asymmetric induced angle of attack distribution. The following distribution for the eight-element wing was arbitrarily selected:

$$
9^{\circ}, 9^{\circ}, 9^{\circ}, 9^{\circ}, 3^{\circ}, 3^{\circ}, 3^{\circ}, 3^{\circ}
$$

The starting distribution for each subsequent interval of the trajectory was assumed to be the previous converged induced angle solution. As evidenced by the time histories of the lateral variables (Fig. 15.b), 
the trajectory clearly describes a wing drop departure to the right, the aircraft rolling through $90^{\circ}$ in about 2.4 seconds. This poststall rolloff motion is characteristic of the full scale Yankee aircraft. Reference 2 discusses the flight test results of power-off, unaccelerated, symmetric stall penetration: "The basic airplane stall was characterized by a roll-off tendency before the elevator could be deflected full up. Full-up elevator could not be held even for a short time without experiencing a roll-off tendency that would, in most cases, lead to an incipient spin."

The longitudinal variables (Fig. 15.a) exhibit the same characteristics as those of the symmetric flight shown in Figure 14 for the initial 2 seconds of the simulation. This is the point at which longitudinal/lateral coupling begins due to the roll-off. As the aircraft rolls past 300 bank attitude at about 3.1 seconds into the maneuver, the body-normal velocity component is reduced as the lateral component increases. This results in angle of attack reduction but also rapid pitch rate increase due to local angle of attack reduction at and consequential unloading of the horizontal tail. The pitch rate drives the angle of attack back toward stall. The rotation of the total lift vector from the vertical accounts for an increase in lateral velocity simultaneously with an increase in descent velocity since it no longer equilibrates the vehicle's weight. A marked pitch attitude decrease is due to the large yaw rate sustained during the steep bank attitude. The yaw rate is set up mostly by the force acting on the vertical fin developed by the aircraft's banked vertical descent. This force is 
indicated by the large values of sideslip angle (Fig. 15.b). The oscillatory roll rate evident in the last second of the trajectory is of the same period as the angle of attack oscillation again demonstrating motion cross-coupling. Finally, the physical geometry of the departure is pictured by the altitude vs. cross range plot of Figure 15.c. This graph shows the rapid loss of altitude characteristic of wing drop departures.

Several observations should be made regarding the two flight simulations discussed above, subject, of course, to the configuration investigated here. Multiple solutions to the lifting line equations exist and their influence in trajectory shaping is strong enough to yield either lateral stability (Fig. 14) or instability (Fig. 15) results. The possibility of a wing drop departure from symmetric flight conditions prior to the stall penetration has been verified without the supposition of an initial rolling or yawing velocity. Since the symmetric stall mode has not been identified in flight testing, it is expected that this dynamic solution is highly unstable. Finally, the effect of pitch-up dynamics is very important to departure prediction. Initiation of the asymmetric solution search just as one or more wing elements begin to stall results in the establishment of an asymmetric lift distribution before maximum $\alpha$ is obtained. However, the duration of the associated rolling moment is too short to produce the roll-off. As the aircraft pitches up into deep stall (all wing elements stalled) the asymmetric distribution virtually vanishes. The large, sustained rolling moments occur in the asymmetric unstalling of the wing vortex segments. This 
dependence on pitch-up dynamics was also detected in a simulation (not reproduced here) in which the aircraft was trimmed at an angle of attack such that the wing was partially stalled. The asymmetric induced angle distribution was implemented, an asymmetric lifting line solution was converged but instead of an abrupt wing drop, the aircraft rolled off in a very gentle spiral motion. Once established, the asymmetric stall pattern did not change. The proper inclusion of vehicle dynamics prior and subsequent to stall penetration is necessary for the prediction of departure characteristics.

In order to examine the effects of flight asymmetries on poststall departure characteristics and determine whether the asymmetric stall pattern could be triggered by slight out-of-trim conditions, a trajectory simulating an initial $-5^{\circ}$ sideslip angle is presented in Figure 16. No artificial asymmetric solutions are introduced; the starting angle-of-attack distributions for each time interval are assumed equal to the previous converged solutions. The result is an abrupt break to the left characterized by oscillating angle of attack and rapid accelerations in pitch rate and total velocity (see Fig. 16.a) similar to the motions associated with the symmetric stall break of Figure 15. The roll-off begins at the occurrence of maximum angle of attack during the initial stall penetration after 1.2 seconds elapsed time, much earlier than the corresponding 2 second time period of Figure 15. The 0.8 second roll commencement lead in Figure 16 accounts for the larger magnitudes recorded in pitch, yaw and bank attitude at the end of the simulation. Examination of the wing stall patterns during 
the stall pitch-through indicates the reason for the earlier onset of roll-off. The left-wing semispan, yawed into the flight path direction, stalls completely during the initial pitch-up while the right wing-tip vortex element, yawed out of the flow, never stalls during the maneuver. This sets up a very large negative roll moment at the stall break resulting in the abrupt left wing drop. The sideslip oscillation recorded in Figure 16.b is due to the aircraft's initial tendency to zero the asymmetry (wethercock stability) followed by a large negative increase in slip from the left roll-off motion and banked descent. The return toward zero sideslip at the end of the trajectory results from an apparent exit or "diving out" maneuver from the departure.

It is noted that the left-hand break illustrated by the simulation is opposite that expected from full scale flight experience. The leeward wing, in this case the right wing, usually stalls ahead of the windward wing because of separated flow induced by crossflow over the fuselage. The result is a positive roll moment producing a right wing drop. Since fuselage effects are not represented in the present model, the physical situation could not be exactly simulated. Still, the computational results are sufficient to conclude that small flight asymmetries prior to stall can induce abrupt departure. The associated altitude loss is presented in Figure 16.c.

An example of how this dynamic model might be used in the analytical investigation of passive "aerodynamics methods for preventing the stall departure" (Ref. 1) is shown in the trajectory of Figure 17. The flight scenario is identical to that of Figure 15 except that the two outer wing 
elements on either semispan are assigned the drooped leading edge lift characteristics of Figure 8 . The longitudinal variables (Fig. 17.a) exhibit the same short period oscillation recorded for the symmetric stall case (Fig. 14) however, the amplitude decay is much more pronounced. As the aircraft penetrates the stall, the inboard unmodified wing section stalls before the outboard drooped panels. The result is a reduction in positive pitch moment allowing the aircraft to dive and unload the wing. The unstalled wing then re-establishes the pitch-up moment and the cyclic motion repeats. As in the case of Figure 14, the increase in velocity during the last half of the trajectory signals the development of a moderate sink rate. Even with the introduction of the asymmetric induced angle-of-attack distribution which led to the rolloff departure in Figure 15, the lateral variables show no abrupt wing drop (Fig. 17.b). Since the outboard vortex elements never stall and are the dominating influence on lateral stability, the small rolling moments set up by inboard asymmetric stalling are insufficient to produce a departure. The small fluctuations of the lateral variables are due to the stalling of the unmodified wing elements. The maximum rolloff in the 5 -second flight is only $3^{\circ}$ to the left while yaw excursion is contained between $+1^{\circ}$ and $-1^{\circ}$.

The beneficial poststall effects of the drooped leading edge configuration shown in Figure 17 are clearly indicated by flight test experience: "In contrast to the stall behavior with the basic wing, no roll-off is noted throughout the period the elevator was deflected full up (for the modified outboard leading edge). Instead, a slight 
wing rock developed ..." The pilot noted in some cases, "the airplane exhibited no roll-off tendency whatsoever and entered a wings-level highsink-rate flight condition with full aft stick." (Ref. 2) 


\section{CONCLUSIONS AND RECOMMENDATIONS}

A dynamic model applicable to the analysis of poststall flight characteristics has been developed and applied to the prediction of wing drop departures of general aviation aircraft. The model consists of the integration of a computational aerodynamics package with a six degree-of-freedom flight simulator. The aerodynamics generator is based on a nonlinear lifting line theory with an unsteady, nonplanar vortex wake applied to each lifting surface of the aircraft (wing, horizontal tail and vertical fin). Trajectory simulation is accomplished through digital integration of the nonlinear rigid-body equations of motion. The results are presented as time histories of flight parameters which completely describe the vehicle trajectory. Data presented herein support the following conclusions:

1. A model representing the interaction of aerodynamics with vehicle dynamics is necessary for the investigation of stall departure. The abruptness of poststall wing drop was found to depend on the presence of flight asymmetries at the stall and the rate of stall penetration. Very gradual approach to the stall resulted in a spiral-type roll-off while a violent wing drop developed subsequent to high pitch-rate stall penetration. 
2. Multiple lifting line solutions exist for a discretized wing whose vortex elements are assigned the lift properties of an airfoil having stall discontinuities. These multiple solutions directly affect the vehicle's lateral stability and hence are major factors in shaping the poststall trajectory.

3. One of the lifting line solutions mathematically possible during symmetric penetration of the stall is an asymmetric stall pattern yielding a large rolling moment due to asymmetric lift distribution. The resulting abrupt roll-off prediction correlates well with the known wing-drop departure of trimmed full scale aircraft pitching through the stall.

4. The stability of the multiple lifting line solutions may be investigated by varying the aircraft's flight conditions prior to the stall break. For example, a small prestall sideslip perturbation resulted in the establishment of an asymmetric stall pattern producing a poststal1 departure.

5. The dynamic model can be used in the generation of dynamic stability parameters by analytically simulating forced oscillation tests. Results presented for the generation of the angle-of-attack variation of $C_{\ell p}+C_{\ell_{\dot{\beta}}} \sin \alpha$ by oscillation-in-roll simulations show good agreement with wind tunnel data. 
6. The effects of passive aerodynamic modifications for improved resistance to departure are well predicted by the dynamic model both in forced-roll oscillation calculations and in the flight simulations. The modifications investigated here involved extending the leading edge on the outboard wing panels of the Yankee aircraft; the analysis verified the flight test conclusions that this configuration results in poststall lateral stability.

7. The model was specifically formulated for utilization in the design phase of future configuration development. For example, the aircraft's geometry is completely variable and only a minimal amount of aerodynamic experimental data is required ( 1 ift curve characteristics).

The basic concept of interactive aerodynamics and vehicle dynamics has provided the present model with much flexibility in the analysis of high angle-of-attack flight characteristics. The results show the technique to be very effective in the prediction of poststall lateral instabilities, at least for general aviation configurations. They also encourage the extension of the dynamic model to more detailed analys is of not only the high angle-of-attack regime but of all flight situations which may be anticipated for a particular aircraft during its preliminary design. The following recommendations are suggested for further development of the model: 
1. The modeling of fuselage effects may be accomplished by representing the body according to several methods: a set of plane quadrilaterals with individual source densities chosen so that the normal velocity is zero at a specified control point (Ref. 34); an extended slender body theory approach (Ref. 35); the method of trailing horseshoe vortex images (Ref. 36).

2. The analytical investigation of unconventional aircraft configurations may provide valuable data on their poststall flight charateristics prior to flight or wind tunnel tests. For example, the canard VariEze, V-tail configurations, light twin-engine aircraft and small business-type, straight-wing jets.

3. The effects of configuration modifications for a given aircraft (e.g. the vertical position of the tail relative to the center of gravity) may be studied for the purpose of configuration definition to improve poststall handling.

4. An interesting application of the present model would be its use in analyzing high performance fighter-type aircraft with straight or moderately swept wings (e.g. F5, F16, F18 or F14 with wings swept forward). The asymmetric separation of vortices from the slender pointed noses of these aircraft will have a dynamic influence on the establishment of wing asymmetric stall patterns. (Reference 37 presents a vortex shedding model relevant to this application.) 
5. Similar to the forced-roll oscillation procedure outlined in this paper, the damping-in-yaw parameter $C_{N_{r}}+C_{N_{\dot{B}}} \cos \alpha$ and dampingin-pitch term $C_{M_{q}}+C_{M_{\alpha}}$ can be generated by simulating oscillation about the $z-$ and $y$-axis respectively.

6. By supplying the analytical model with appropriate vehicle motions, the dynamic stability derivatives may be isolated from the acceleration derivatives. For instance, $C_{\ell_{p}}$ and $C_{\ell_{\dot{\beta}}}$ may be solved for separately as opposed to the combination $C_{\ell_{p}}+C_{\ell_{\dot{B}}} \sin \alpha$ found through the oscillation procedure.

7. The influence of simultaneous angle of attack and sideslip combinations on forced oscillation data is easily determined by prescribing pitch and yaw attitudes about which the analytical model is oscillated. For example, a family of $c_{\ell_{p}}+C_{\ell_{\dot{\beta}}}$ sin $\alpha$ curves may be plotted as a function of sideslip angle with angle of attack as parameter, etc.

8. Poststall flight characteristics should be determined for accelerated stall maneuvers (climbing, turning) and compared to the $1 \mathrm{~g}$, wings level stall penetration results.

9. Due to the model's capability for varying the incidence of the horizontal tail, control sequencing for stall exit or spin entry recovery can be simulated. 
10. The possibility of allowing the departure trajectories reported in this paper to develop into steady-state spins should be investigated. One preliminary test case indicates that a substantial increase in velocity severely distorts the one-chord distance desired for the shedding of transverse vortices. A variable time step should be used in the integration scheme.

11. The effect of aileron deflection at the stall break on departure development may be analyzed by using flapped section lift charateristics for the wing-tip vortex elements. The availability of lift data for various flap angles would aid in simulating aileron deployment.

12. Basic research into the multiple lifting line solution phenomena should be conducted to determine how many exist for a given configuration and the stability associated with each solution.

13. At the expense of computation time, the model may be fine tuned by representing the lifting surfaces and trailing wakes with a finer discretization of vortex elements. This should result in a more realistic representation of the stall cell phenomena.

14. The modeling of chordwise propagation of the stall requires an unsteady, nonlinear lifting surface theory with multiple spanwise lifting lines. Perhaps, this may yield the ultimate stall departure model. 


\section{REFERENCES}

1. NASA Langley Research Center, "Summary of 1980 General Aviation Sta11/Spin Workshop", September 1980.

2. NASA Langley Research Center, "Exploratory Study of the Effects of Wing-Leading-Edge Modifications on the Stall/Spin Behavior of a Light General Aviation Airplane", NASA TP 1589, December 1979.

3. Hreha, Mark A., and Lutze, Frederick H., "Linear Analysis of Poststall Gyrations", Journal of Aircraft, Vol. 17, No. 10, October 1980, pp. 727-733.

4. Anglin, Ernie L., "Aerodynamic Characteristics of Fighter Configurations During Spin Entries and Developed Spins", Journal of Aircraft, Vol. 15, No. 11, November 1978, pp. 769-776.

5. Kroeger, R. A., and Feistel, T. W., "Reduction of Stall-Spin Entry Tendencies Through Wing Aerodynamic Design", SAE Paper 760481, Apri1 1976.

6. Ericsson, L. E., and Reding, J. P., "Analytic Prediction of Dynamic Stal1 Characteristics", AIAA Paper 72-682, June 1972.

7. Strickland, J. H., Oler, J. W., Im, B. J., and Smith, T. G., "A Prel iminary Dynamic Stali Model Using a Vortex Panel Method", AIAA Paper 81-2584, December 1981.

8. Ericsson, L. E., and Reding, J. P., "Unsteady Airfoil Sta11, Review and Extension", Journal of Aircraft, Vo1. 8, August 1971, pp. 609-616.

9. Stough, H. Paul, III, "Flight Test Results for Three Test Aircraft and Basic System Variables", NASA Langley Research Center General Aviation Stal1/Spin Workshop, September 1980.

10. Stough, H. Paul, III, and Patton, J. M., Jr., "The Effects of Configuration Changes on Spin and Recovery Characteristics of a Low-Wing General Aviation Research Airplane", AIAA Paper 79-1786, August 1979.

11. Stickle, Joseph W., "Preface", NASA Langley Research Center General Aviation Stal1/Spin Workshop, September 1980. 
12. Silver, Brent W., "Statistical Analysis of General Aviation Stall Spin Accidents", SAE Paper 760480, April 1976.

13. Levinsky, E. S., "Theory of Wing Span Loading Instabilities Near Sta11", AGARD-CP-204, Prediction of Aerodynamic Loading, 1976.

14. Sivells, James C., and Neely, Robert H., "Method for Calculating Wing Characteristics by Lifting Line Theory Using Nonlinear Section Lift Data", NACA Report No. 865, 1947.

15. Sears, W. R., "Some Recent Developments in Airfoil Theory", Journal of Aerospace Sciences, Vol. 23, No. 5, May 1956, pp. 490-49.9.

16. Phlips, P. J., East, R. A., and Pratt, N. H., "An Unsteady Lifting Line Theory of Flapping Wings with Application to the Forward Flight of Birds", Journal of Fluid Mechanics, Vol. 112, November 1981, pp. 97-125.

17. Patton, James M., Jr., "Flight Test Operations and Objectives", NASA Langley Research Center General Aviation Stall/Spin Workshop, September 1980.

18. Brown, Philip. W., "The Test Pilot's Role in General Aviation Stall/Spin Flight Testing", NASA Langley Research Center General Aviation Stall/Spin Workshop, September 1980.

19. Burk, Sanger M., Jr., and Wilson, Calvin F., Jr., "RadioControlled Model Design and Testing Techniques for Stall/ Spin Evaluation of General-Aviation Aircraft", NASA TM 80510, Apri1 1975.

20. DiCarlo, Daniel J., "Use of Radio-Controlled Models in Stall/ Spin Flight Testing", NASA Langley Research Center General Aviation Stall/Spin Workshop, September 1980.

21. Bowman, James S., Jr., "Evaluation of the Spin Tunnel for General Aviation Testing", NASA Langley Research Center General. Aviation Sta11/Spin Workshop, September 1980.

22. White, Frank M., Viscous Fluid Flow, McGraw Hi11, New York, 1974.

23. Bihrle, William, "Rotary-Balance Force Tests and Analys is of Equilibrium Spin Modes", NASA Langley Research Center General Aviation Stall/Spin Workshop, September 1980. 
24. McCain, Charles, E., Jr., "Utilization of Aerodynamic Coefficients Generated by Pure Rolling and Pure Yawing Flow in the Determination of Aircraft Equilibrium Spin Properties", Master's Thesis, Virginia Tech, June 1978.

25. Tischler, M. B., and Barlow, J. B., "Determination of the Spin and Recovery Characteristics of a General Aviation Design", Journal of Aircraft, Vol 18, No. 4, April 1981, pp. 238-244.

26. Hreha, Mark A., "Linear Analysis of Incipient Spin Dynamics", Master's Thesis, Virginia Tech, June 1979.

27. Bihrle, W., Jr., Hultberg, R. S., and Mulcay, W., "Rotary Balance Data for a Typical Single Engine Low-Wing General Aviation Design for an Angle-of-Attack Range of 30 to 90 ", NASA CR 2972, 1978.

28. Bihrle, William, Jr., "Rotary Balance Data for a Typical SingleEngine General Aviation Design for an Angle-of-Attack Range of $8^{\circ}$ to $35^{\circ}$ III - Effect of Wing Leading-Edge Modifications Model A", NASA CR-3102, November 1979.

29. Abbott, Ira H., and von Doenhoff, Albert E., Theory of Wing Sections, Dover Publications, Inc., New York, 1959.

30. Johnson, J. L., Jr., Newsom, W. A., and Satran, D. R., "FullScale Wind-Tunnel Investigation of the Effects of Wing LeadingEdge Modifications on the High Angle-of-Attack Aerodynamic Characteristics of a Low-Wing General Aviation Airplane", AIAA Paper 80-1844, August 1980.

31. Bihrle, William, Jr., "Static Aerodynamic Characteristics of a Typical Single-Engine Low-Wing General Aviation Design for an Angle-of-Attack Range of $-8^{\circ}$ to $90^{\circ} "$, NASA CR-2971, July 1978.

32. Chambers, Joseph R., and Grafton, Sue B., "Static and Dynamic Longitudinal Stability Derivatives of a Powered 1/9-Scale Model of a Tilt-Wing V/STOL Transport", NASA TN D-3591, May 1966.

33. Winkelmann, Allen E., "Fluorescent 0il Flow Studies of Stall Development on Wings", NASA Langley Research Center General Aviation Sta11/Spin Workshop, September 1980.

34. Smetana, Frederick 0., Summey, Delbert C., Smith, Neill S., and Carden, Ronald K., "Light Aircraft Lift, Drag, and Moment Prediction - A Review and Analysis", NASA CR-2523, May 1975. 
35. Thomas, James L., and Nerney, Brian, "Aerodynamics of Arbitrary Wing Body Combinations with Vortex Lattice and Slender Body Theory", AIAA Paper 76-198, January 1976.

36. Zlotnick, M., and Robinson, S.W., Jr., "A Simplified Mathematical Model for Calculating Aerodynamic Loading and Downwash for Midwing Wing-Fuselage Combinations with Wings of Arbitrary P1anform", NACA RM L52J27a, January 1953.

37. Spangler, S. B., Perkins, S. C., Jr., and Mendenhall, M. R., "Prediction of Lateral Aerodynamic Loads on Aircraft at High Angles of Attack", AGARD-CP-247, High Angle of Attack Aerodynamics, 1978. 


\section{TABLES}




\section{TABLE I}

\section{AIRCRAFT MASS AND GEOMETRY CHARACTERISTICS}

Weight, $\quad N\left(1 b_{f}\right)$

6863

(1543)

Moments of Inertia, $\quad \mathrm{Kg}-\mathrm{m}^{2}\left(\mathrm{sl}-\mathrm{ft}^{2}\right)$ :
Pitch ( $\left.I_{y}\right)$
$826 \quad(609)$
Roll ( ${ }_{x}$ )
$1010 \quad(745)$
Yaw $\left(I_{z}\right)$
1741 (1284)

Center of Gravity, Percent c

Wing:

Span, $m(f t)$

Spanwise Chord Distribution ( $f t)$

$4.0,4.0,4.0,4.0,4.0,4.0,4.0,4.0$

Dihedral, deg

Incidence, deg

Sweep, deg

0.0

Horizontal Tail:

Span, $m(f t)$

Spanwise Chord Distribution ( $f t$ )

$1.848,2.203,2.559,2.915,2.915,2.559,2.203,1.848$

Dihedral, deg

Incidence, deg variable

Sweep, deg 11.93 
TABLE I - Continued

Longitudinal Distance $(\mathrm{cg}$ to $25 \% \mathrm{c}), \mathrm{m}(\mathrm{ft}) \quad 3.35 \quad(11.0)$

$\begin{array}{lll}\text { Vertical Distance, } m(f t) & 0.0 & (0.0)\end{array}$

Vertical Fin:

Span, $m(f t)$

$1.029 \quad(3.375)$

Spanwise Chord Distribution (bottom to top) ( $\mathrm{ft}$ )

$3.040,2.527,2.015$

Sweep, deg

20.56

Longitudinal Distance ( $\mathrm{cg}$ to $25 \% \mathrm{c}_{\text {bottom panel }}$ ), $\mathrm{m}(\mathrm{ft})$

$3.168 \quad(10.395)$

Vertical Distance (cg to bottom element), $m(f t)$

0.321 
FIGURES 

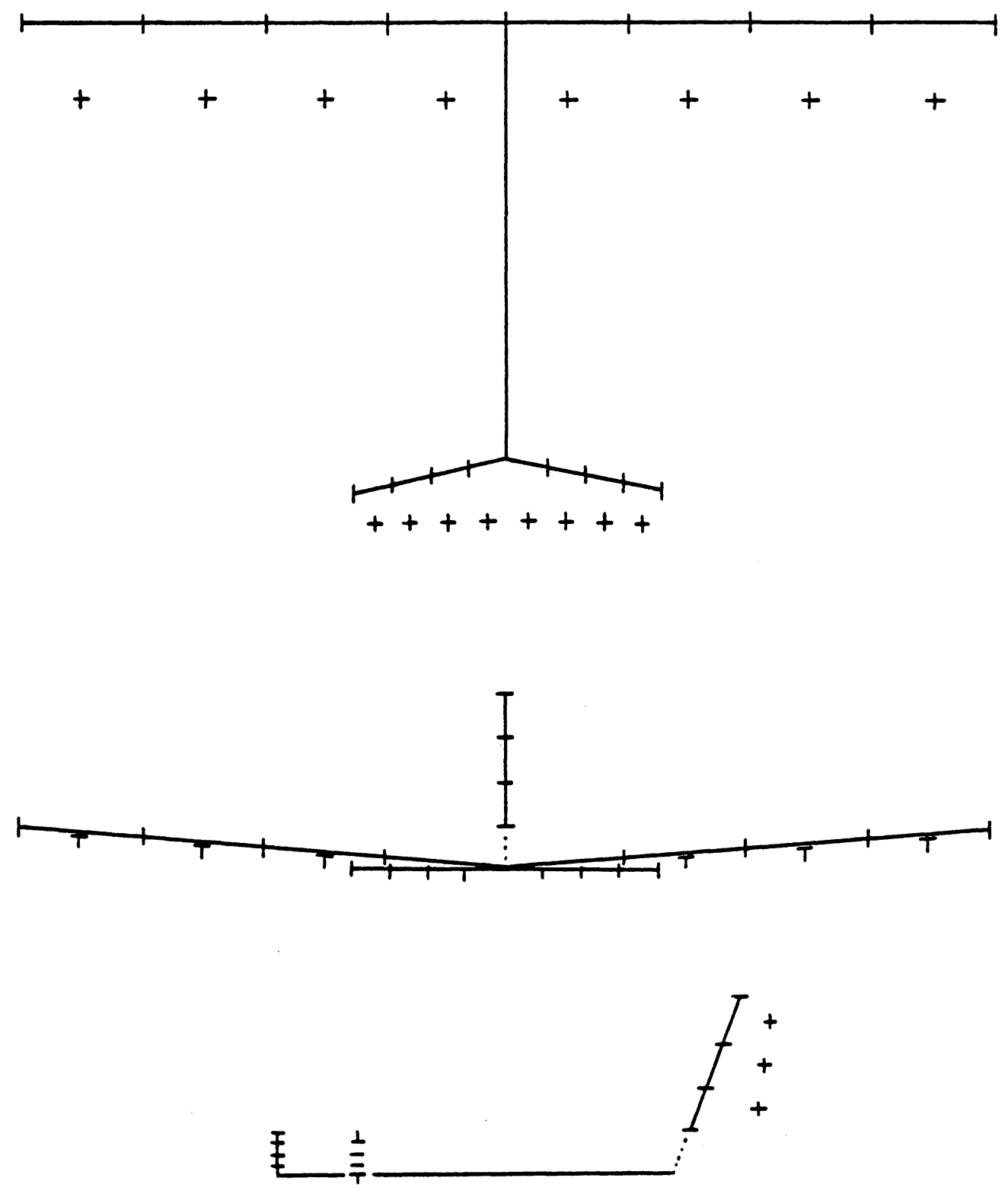

Figure 1 - AIRCRAFT GEOMETRIC MODEL

Discrete Lifting Lines Placed at $25 \%$ Chord of A11 Surfaces Control Points Stationed at $75 \%$ Chord Streamwise 


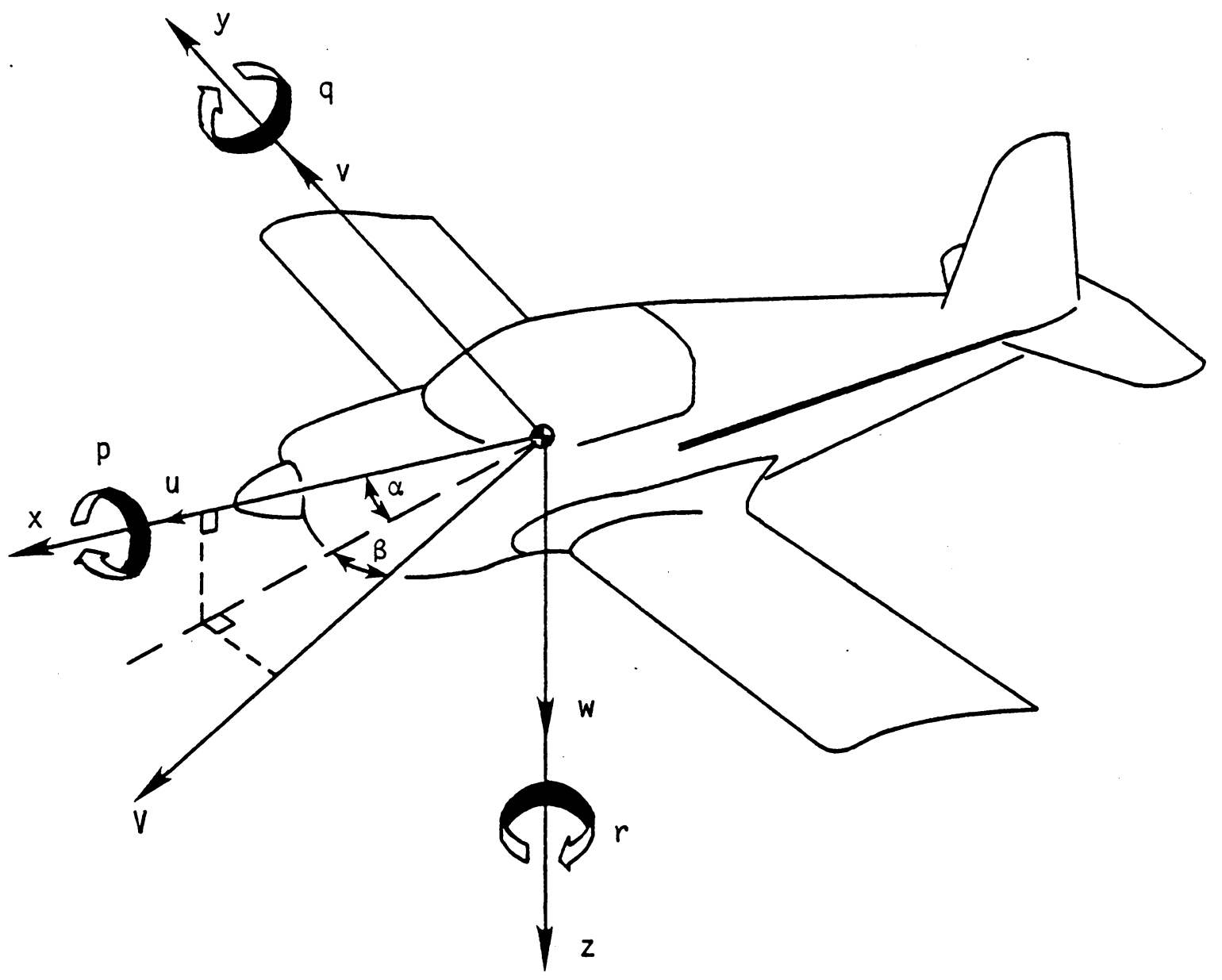

Figure 2 - Body - Axis System 


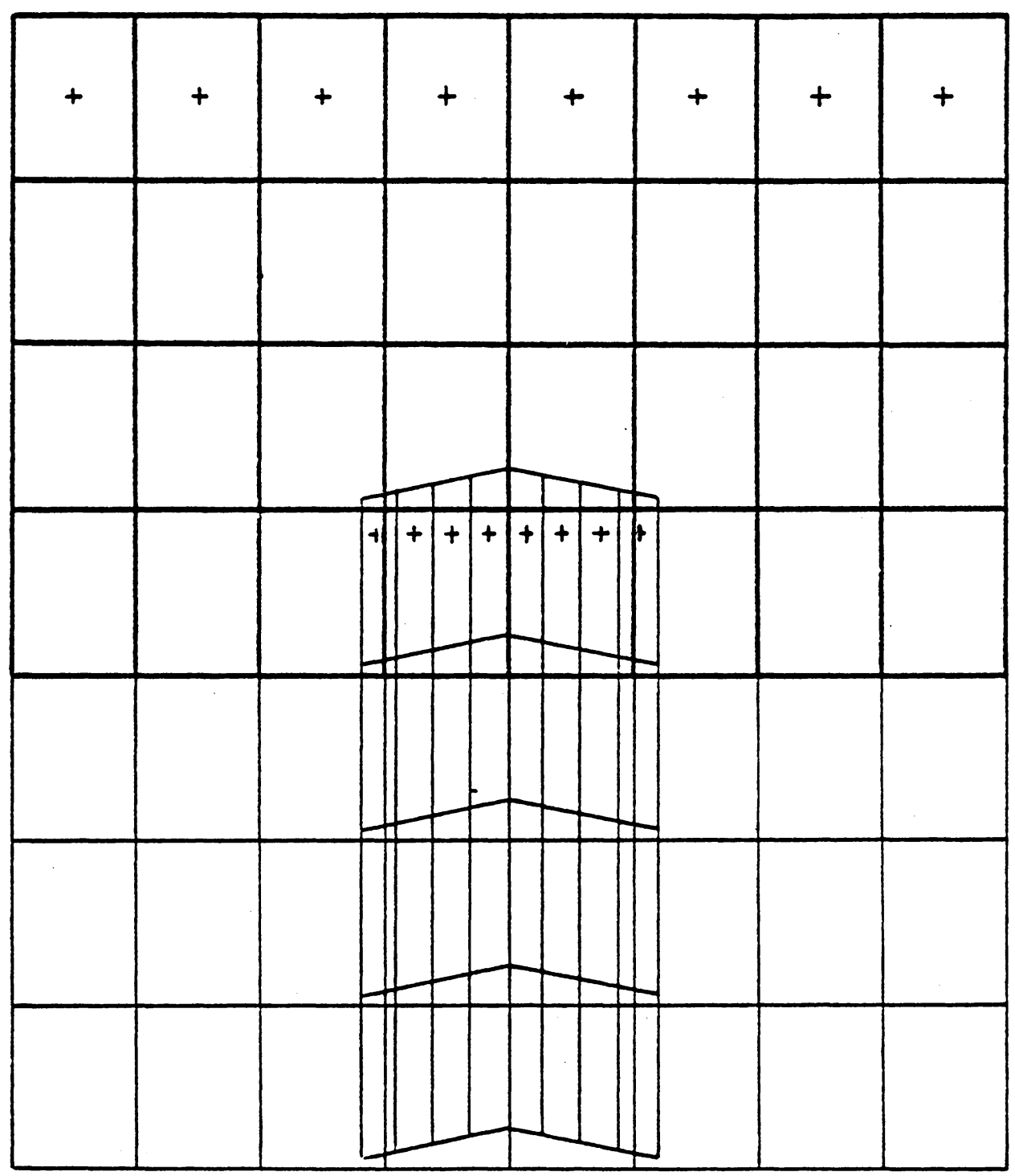

Figure 3 - INITIAL VORTEX SYSTEM GEOMETRY

Ma in Wing Downwash Computed from

8 Spanwise by 4 Streamwise Parallelogram Lattice Elements Horizontal Tail and Vertical Fin Wash Computed from

8 Spanwise by 7 Streamwise Ma in Wing Elements

8 Spanwise by 4 Streamwise Tail Plane Elements

3 Spanwise by 4 Streamwise Vertical Fin Elements 

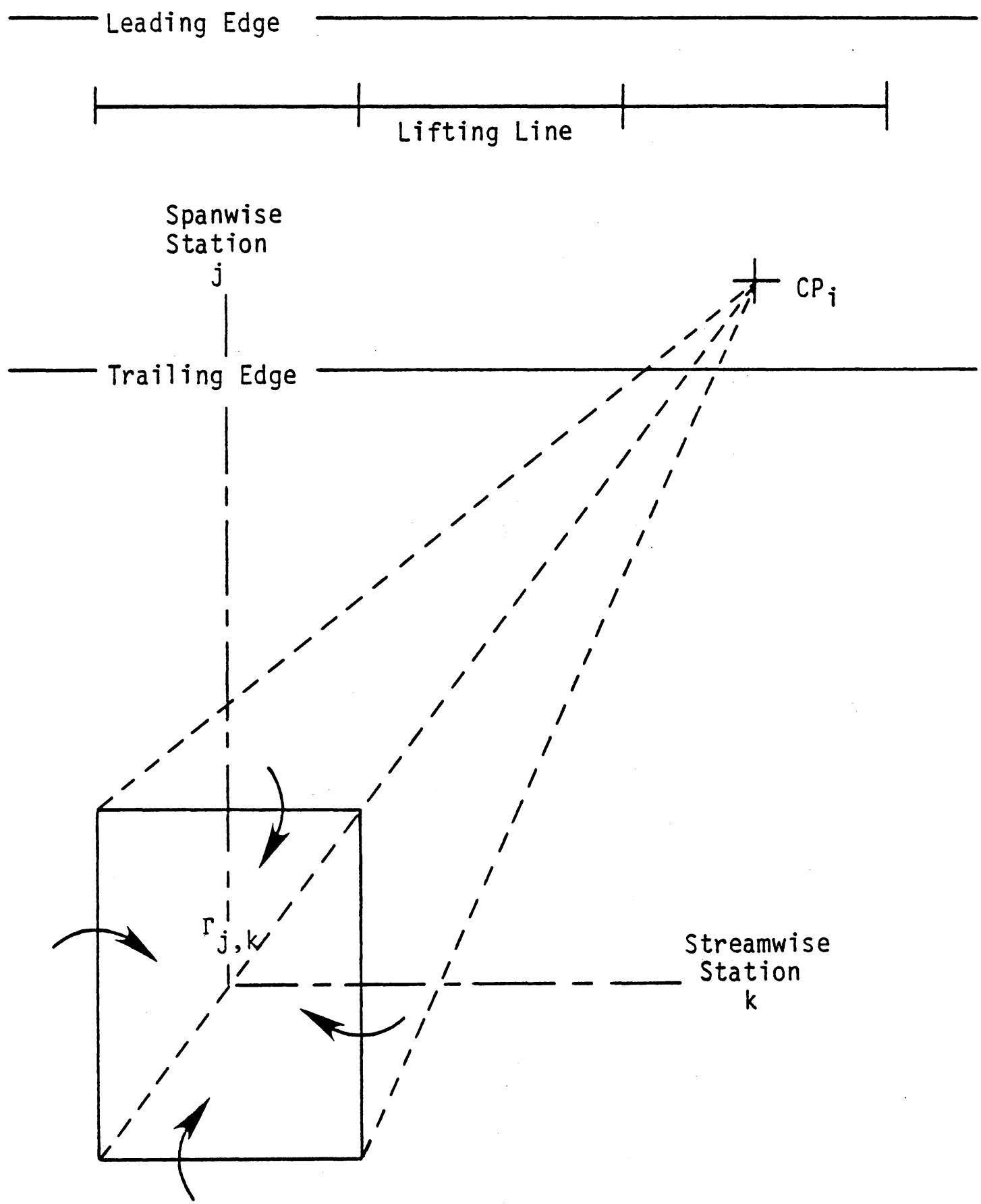

Figure 4 - Geometric Relationship between Control Point $i$ and Parallelogram Lattice Element $j, k$ 


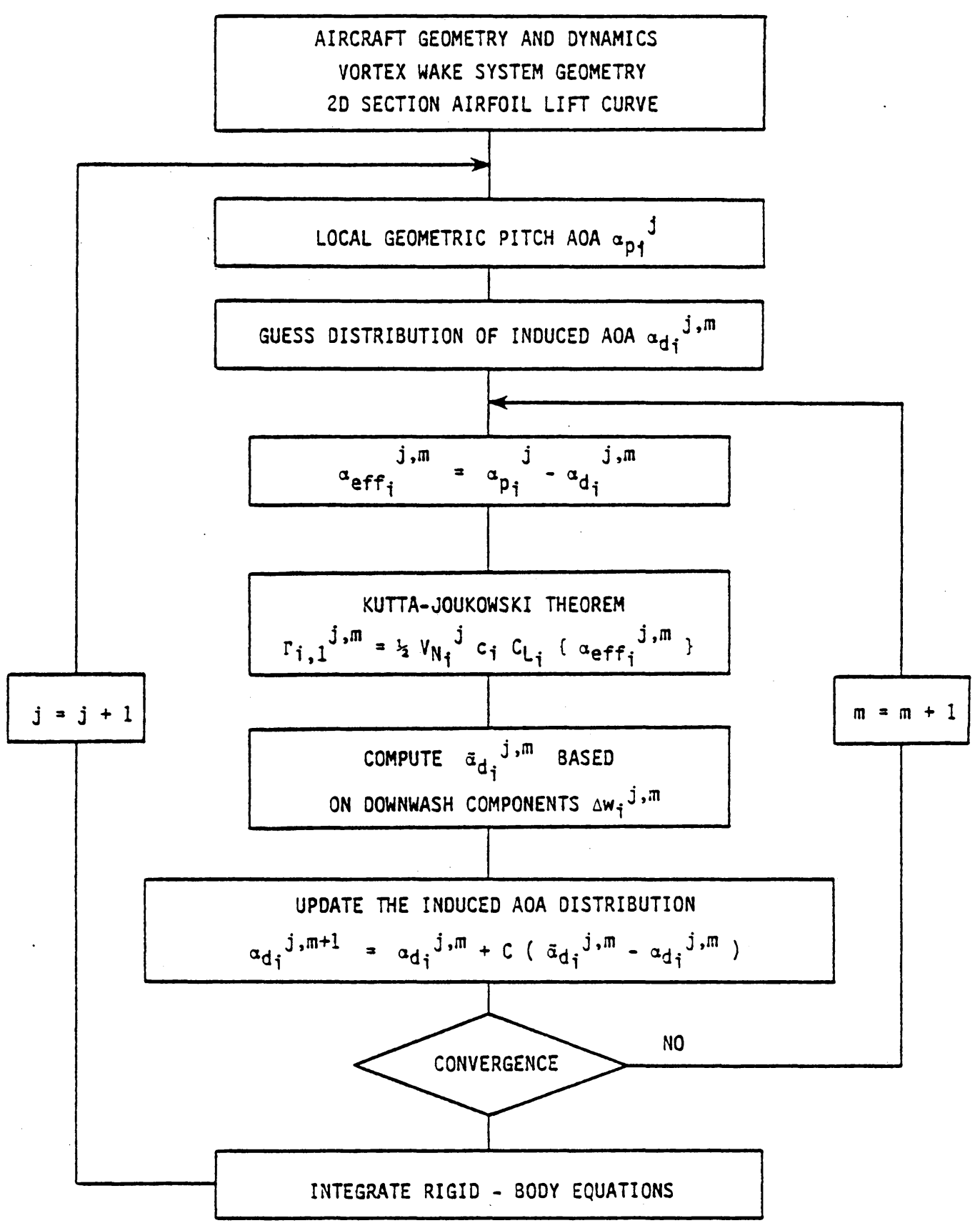

Figure 5 - Dynamic Model Flowchart 


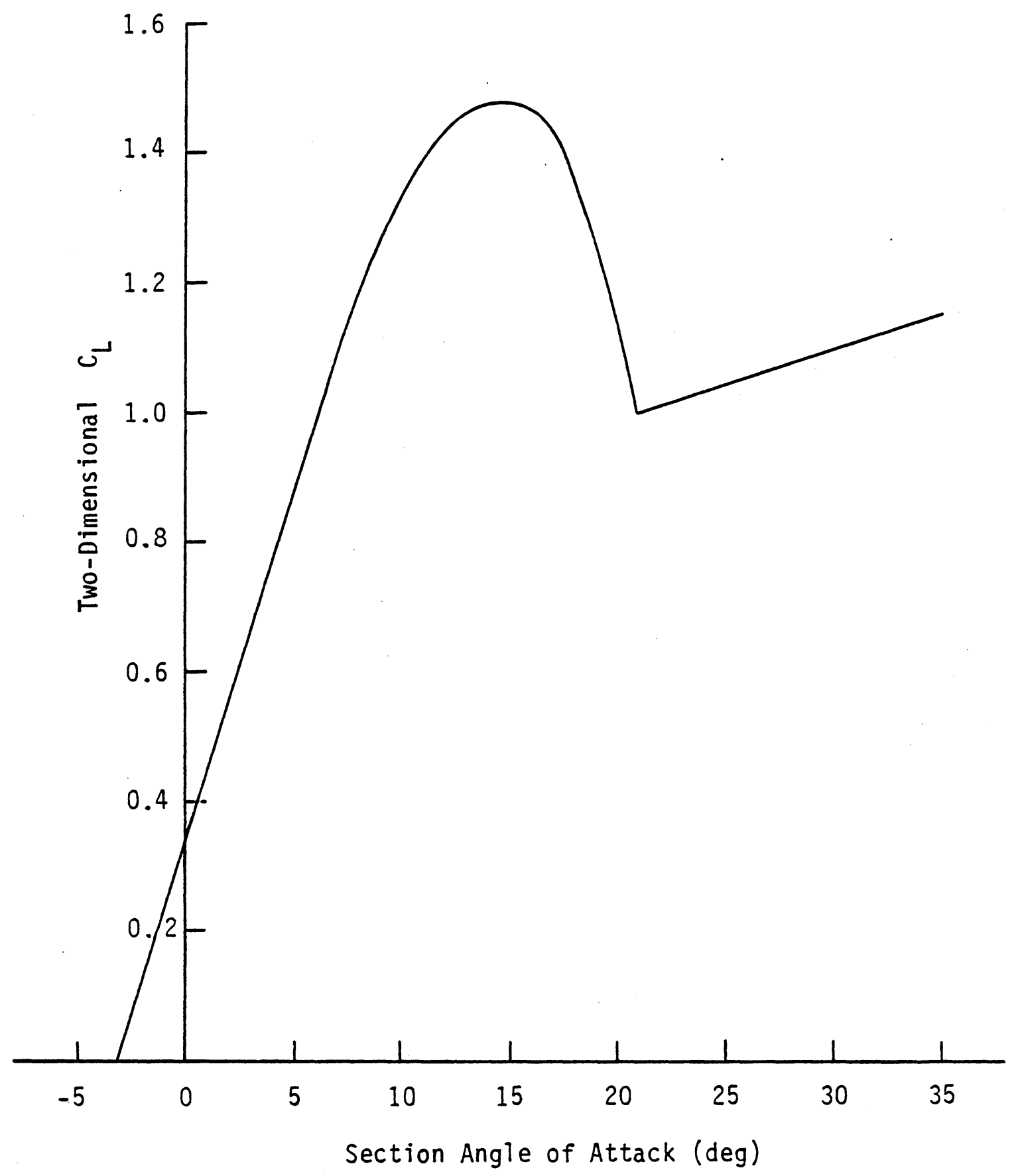

Figure 6 - Lift Curve for Basic 642-415 Airfoil Section 


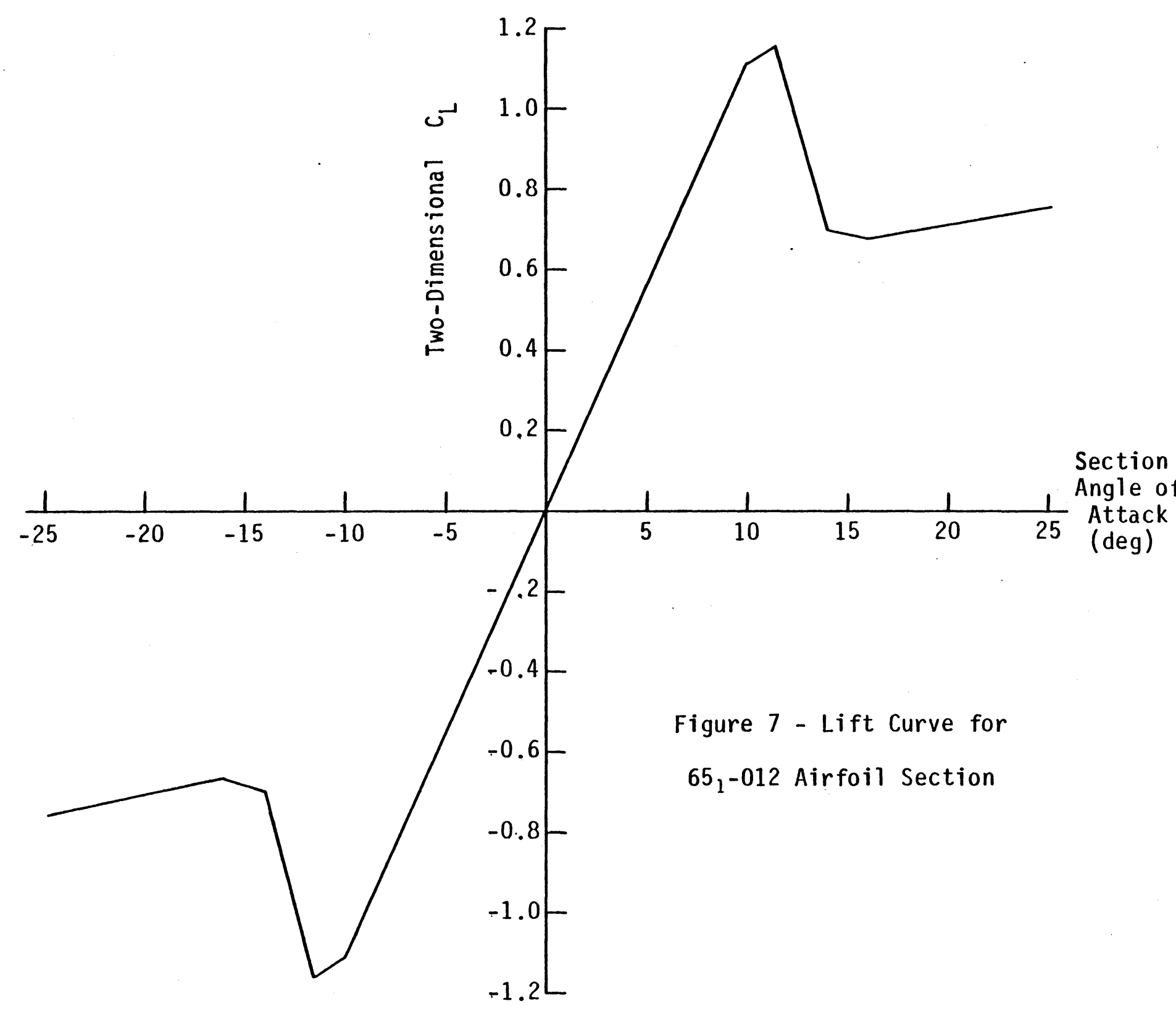




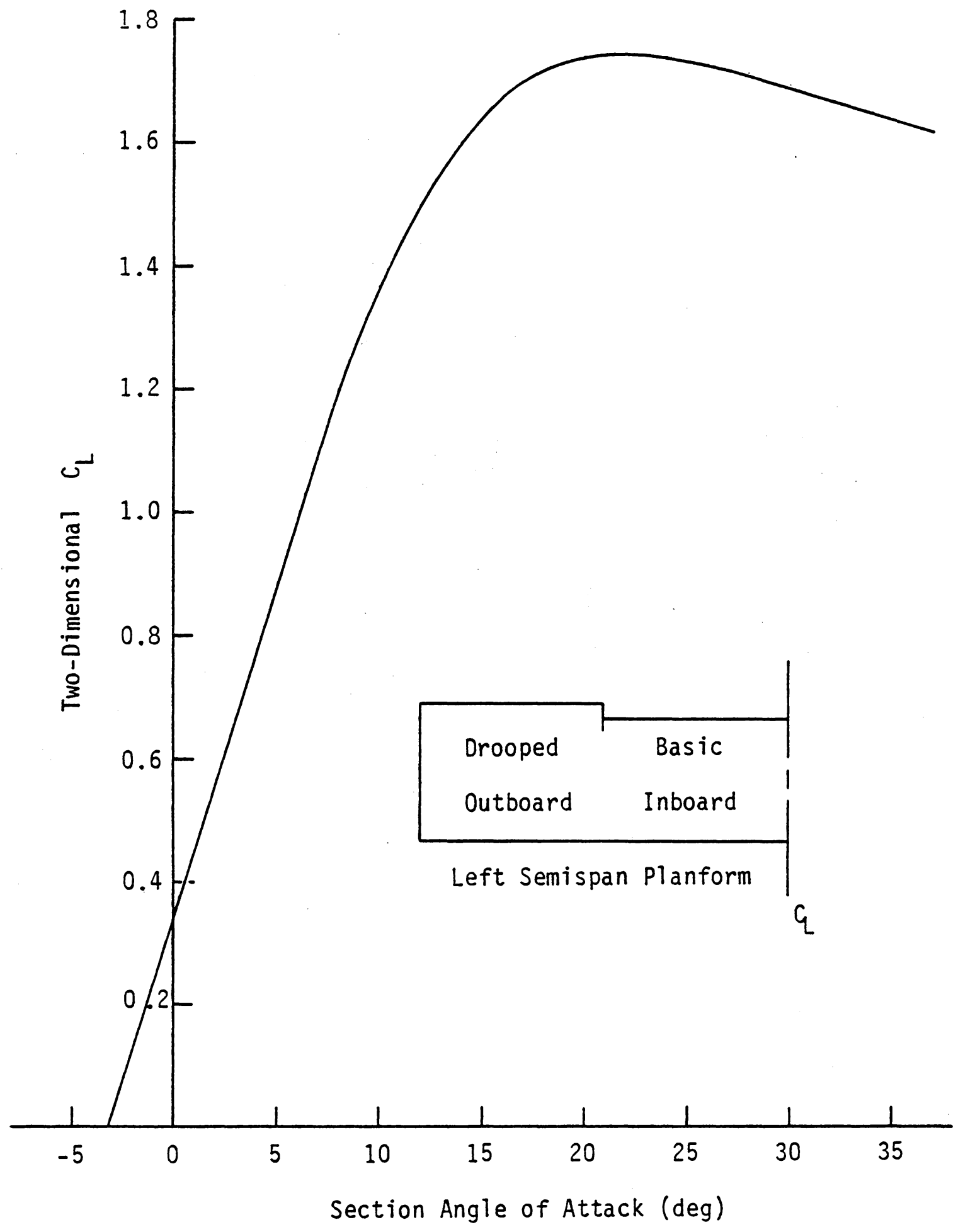

Figure 8 - Lift Curve for $64_{2}-415$ Airfoil Section with Drooped Leading Edge 

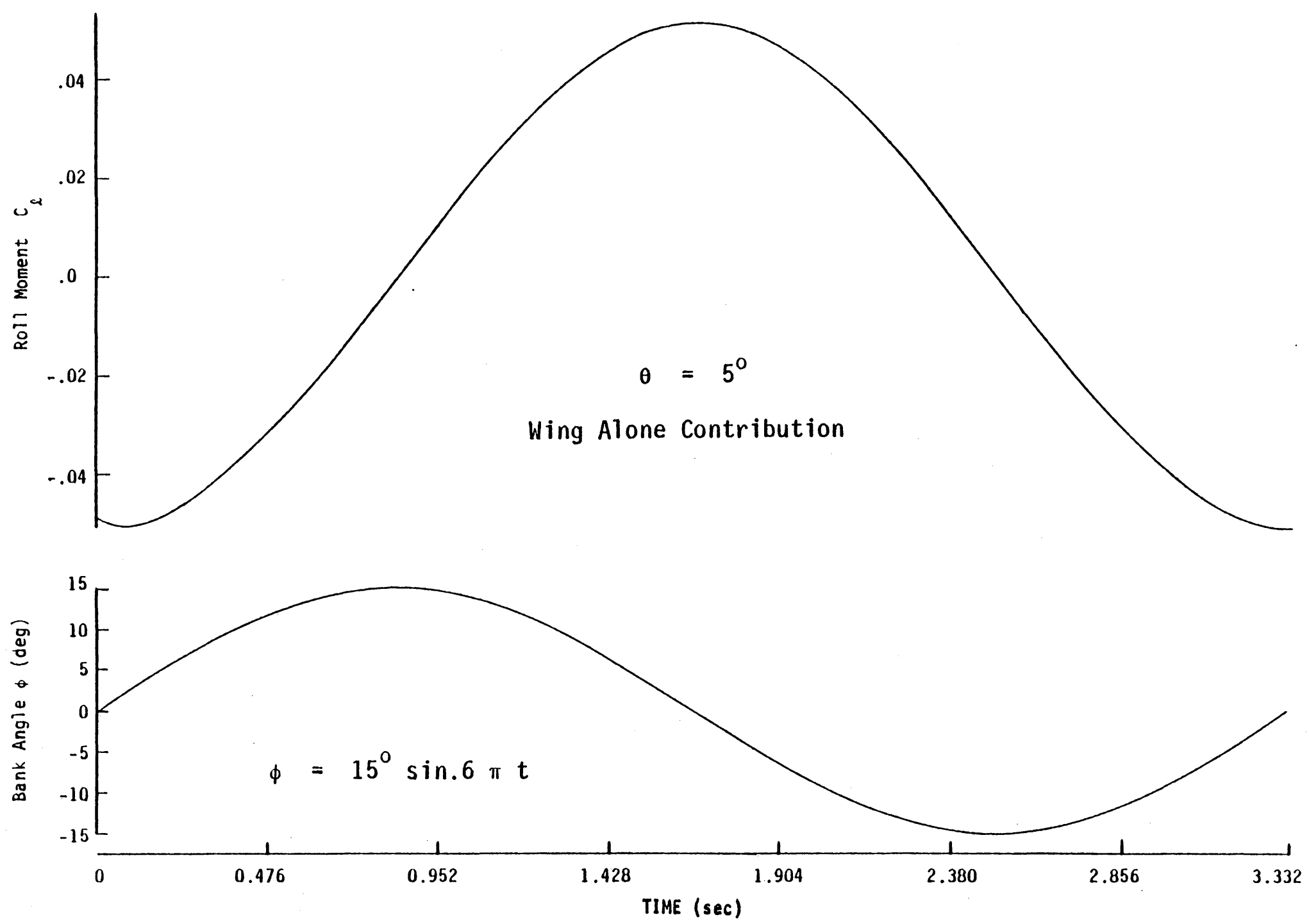

Figure 9 - INPUT AND OUTPUT SIGNALS FOR FORCED-ROLL OSCILLATION SIMULATION 


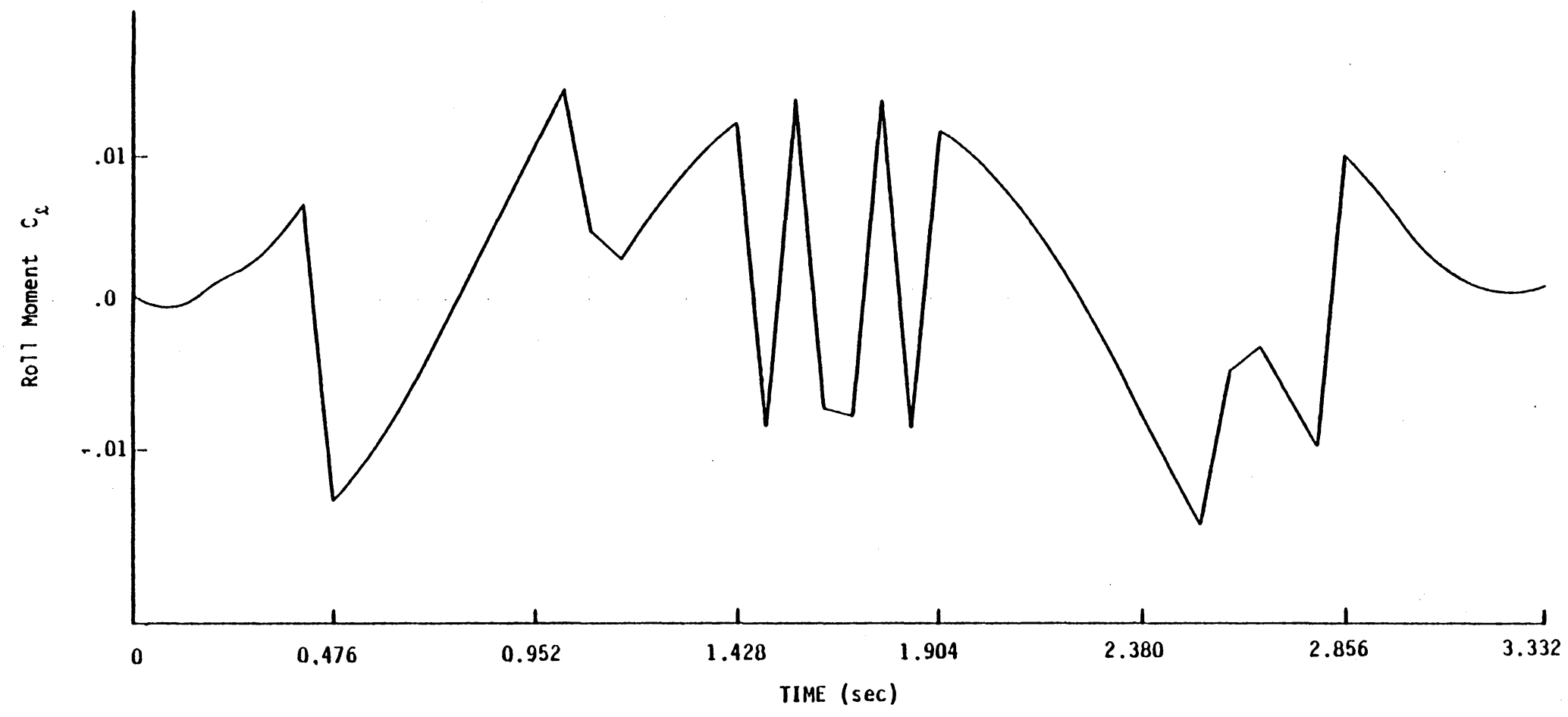

Figure 10 - OUTPUT SIGNAL FOR FORCED-ROLL OSCILLATION SIMULATION $\theta=14^{\circ}$ Wing-Empennage Configuration 


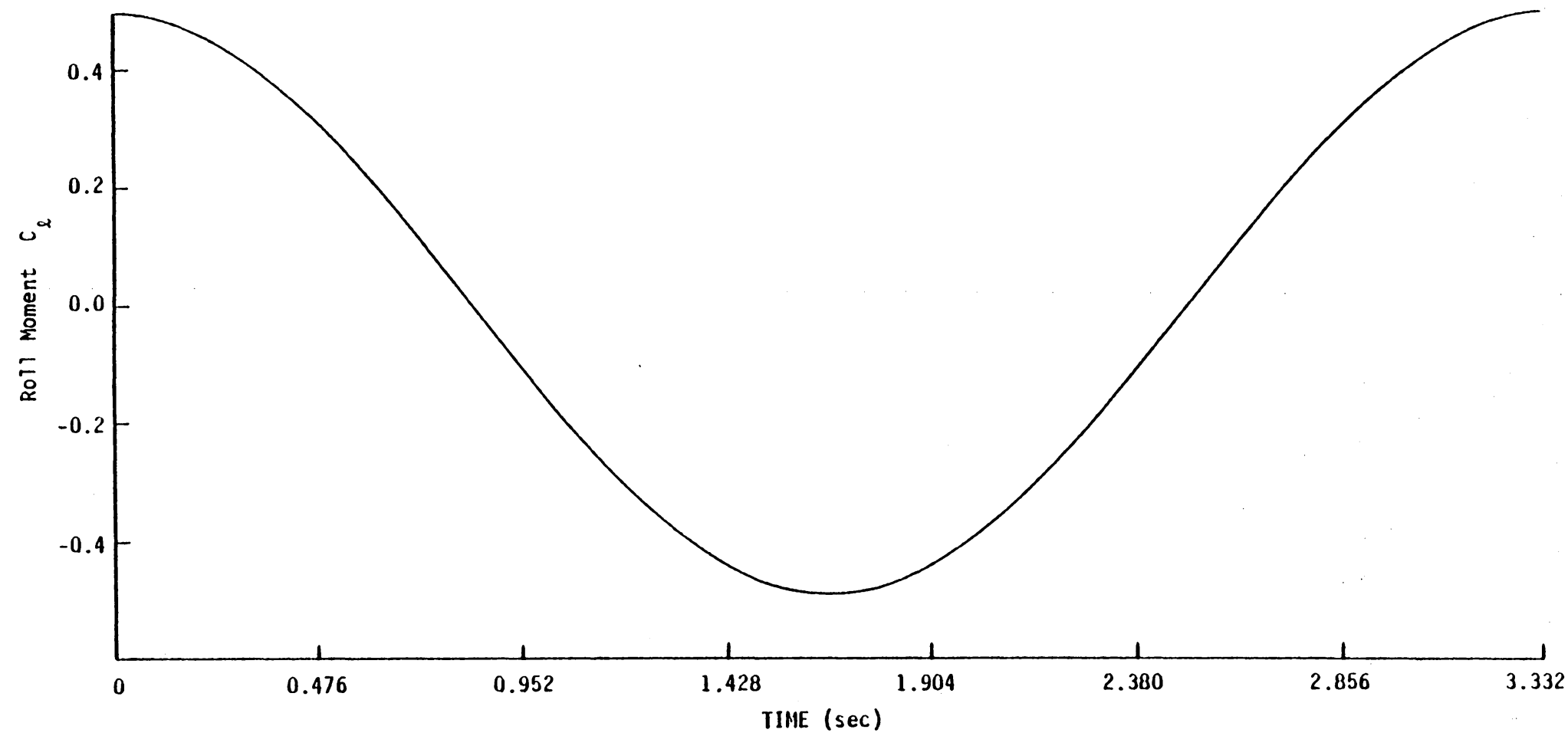

Figure 11 - OUTPUT SIGNAL FOR FORCED-ROLL OSCILLATION SIMULATION $\theta=18^{\circ}$ Wing Alone Contribution 


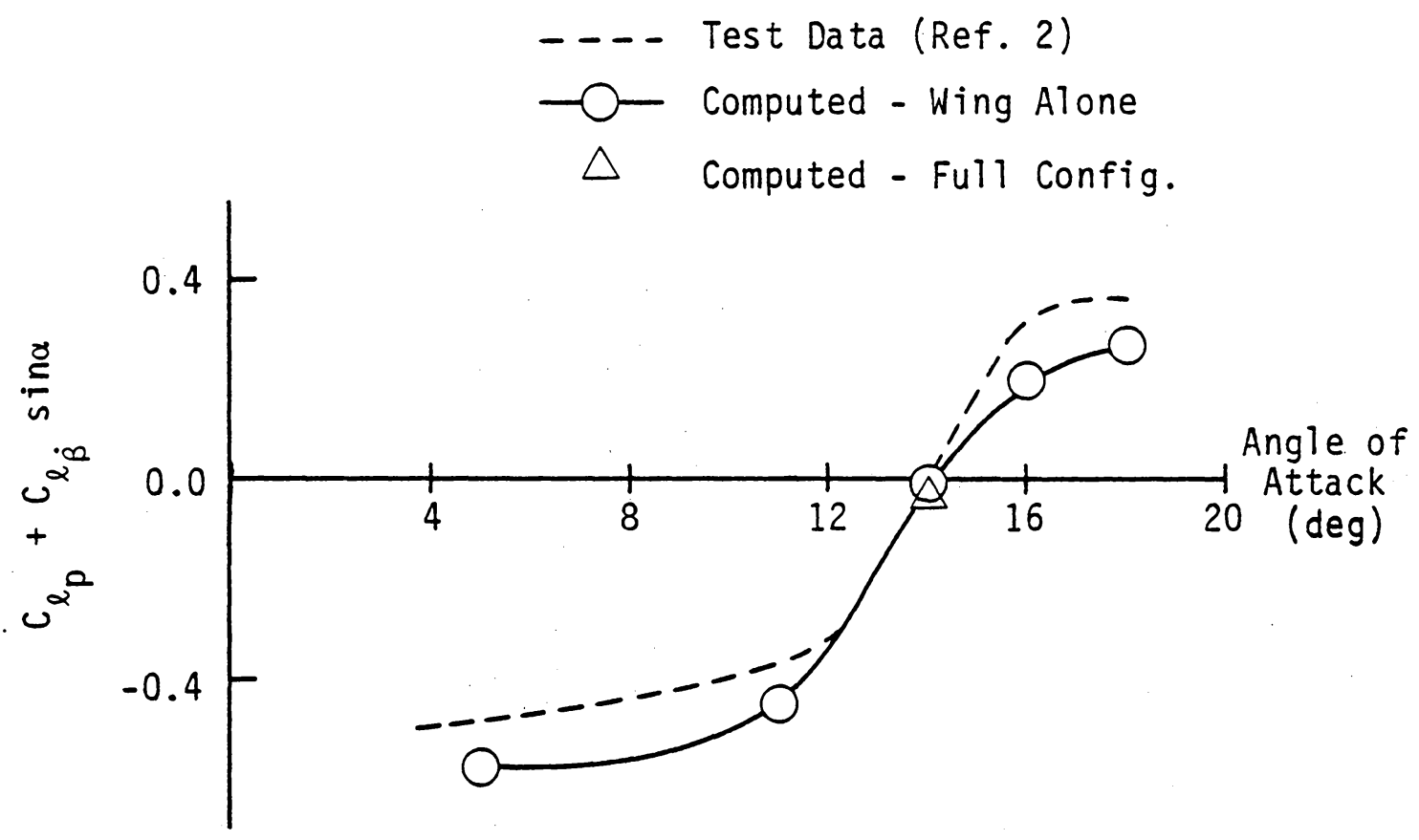

Figure 12.a - Damping-in-Roll Parameter vs Angle of Attack Basic Wing. Amplitude $= \pm 15^{\circ}$. Frequency $=0.3 \mathrm{cycles} / \mathrm{sec}$.

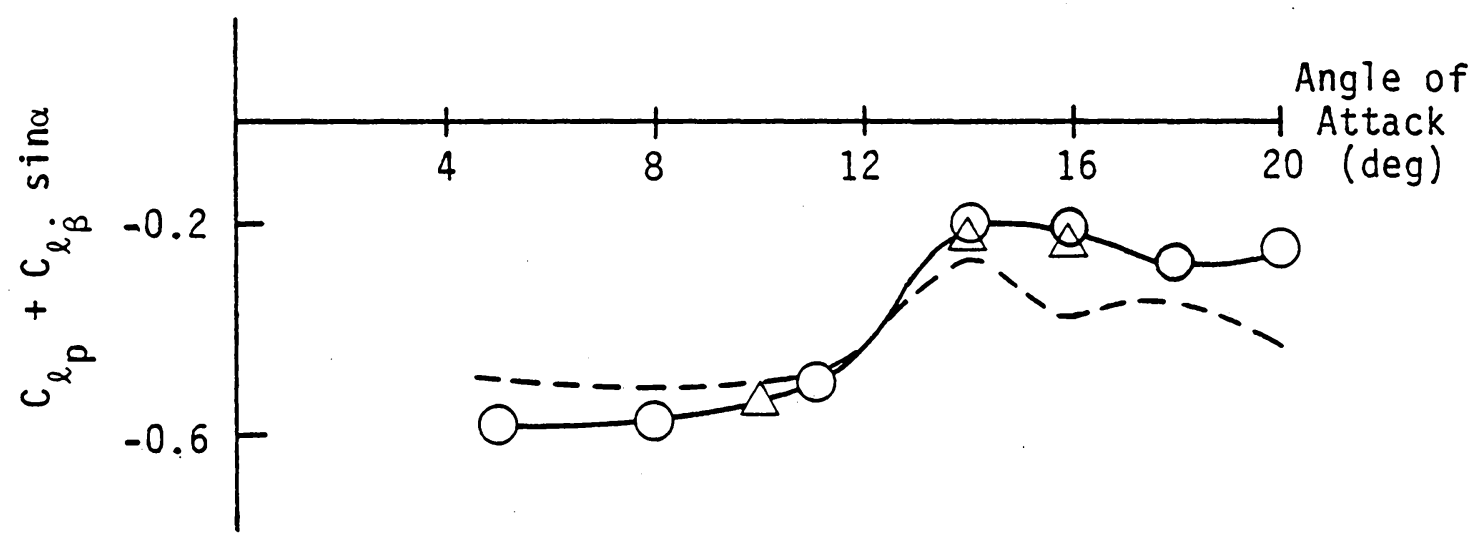

Figure 12,b - Damping-in-Roll Parameter vs Angle of Attack Drooped Outboard Segmented Wing Amplitude $= \pm 15^{\circ}$. Frequency $=0.3 \mathrm{cycles} / \mathrm{sec}$. 
$\left.\right|^{v}$

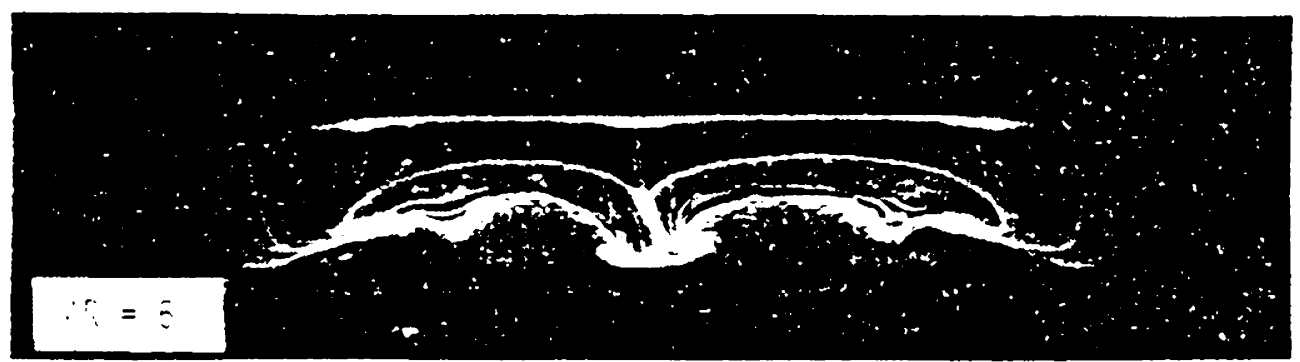

0il Flow Visualization (Ref. 33)

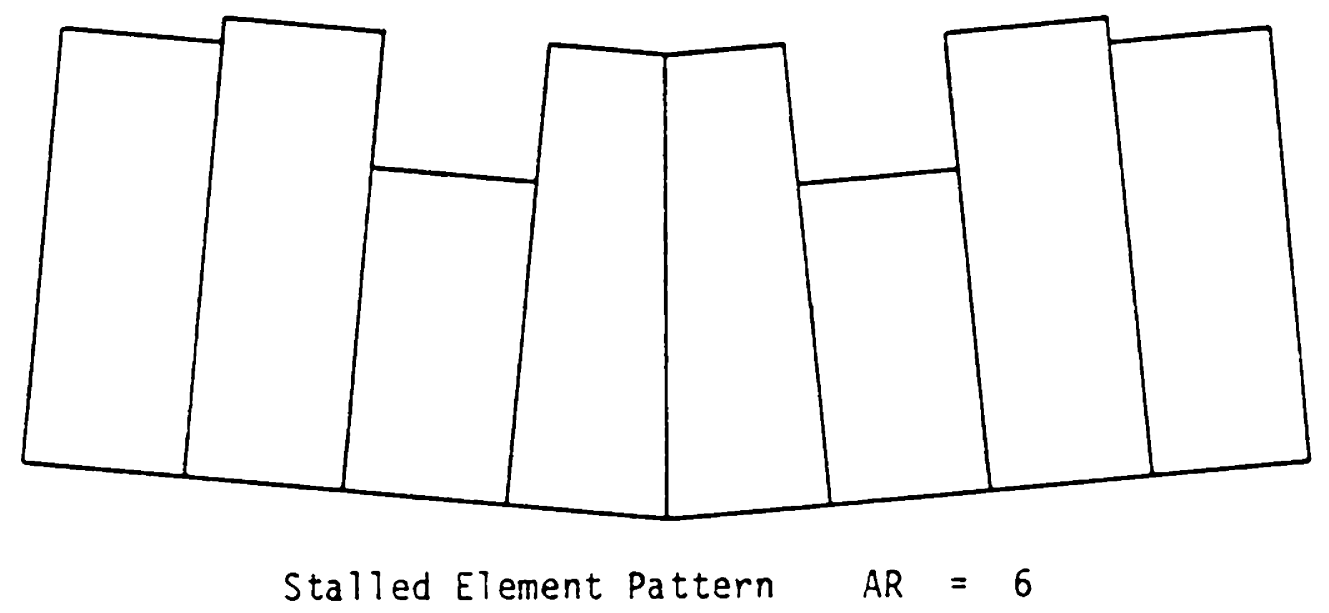

Figure 13 - PHYSICAL AND COMPUTED STALL CELL PATTERNS 

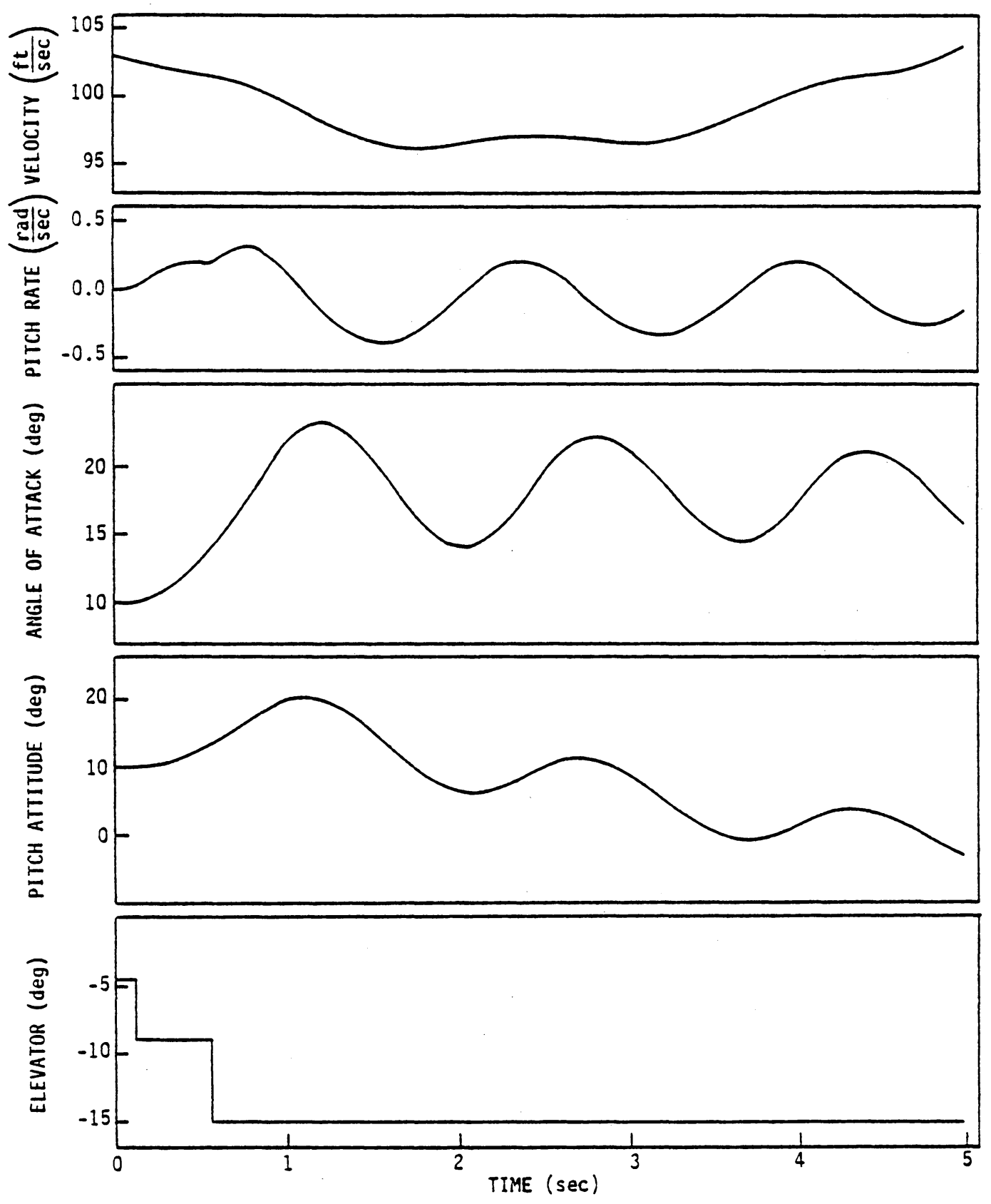

Figure 14 - SYMMETRIC STALL PENETRATION

Basic Wing - Longitudinal Parameters 


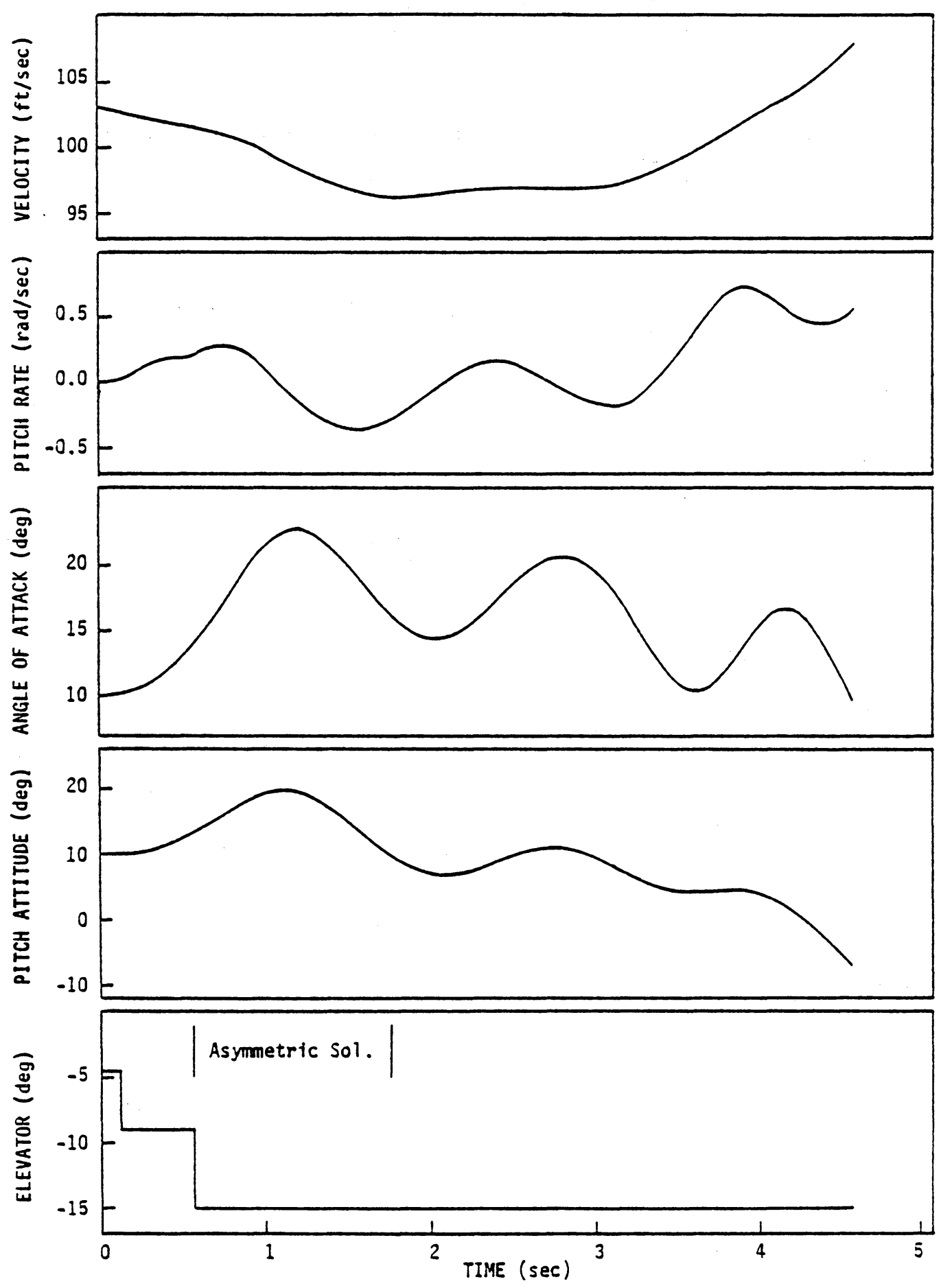

Figure 15.a - SYMMETRIC STALL PENETRATION

Basic Wing - Longitudinal Parameters.

Asymmetric Induced Angle-of-Attack Solution 


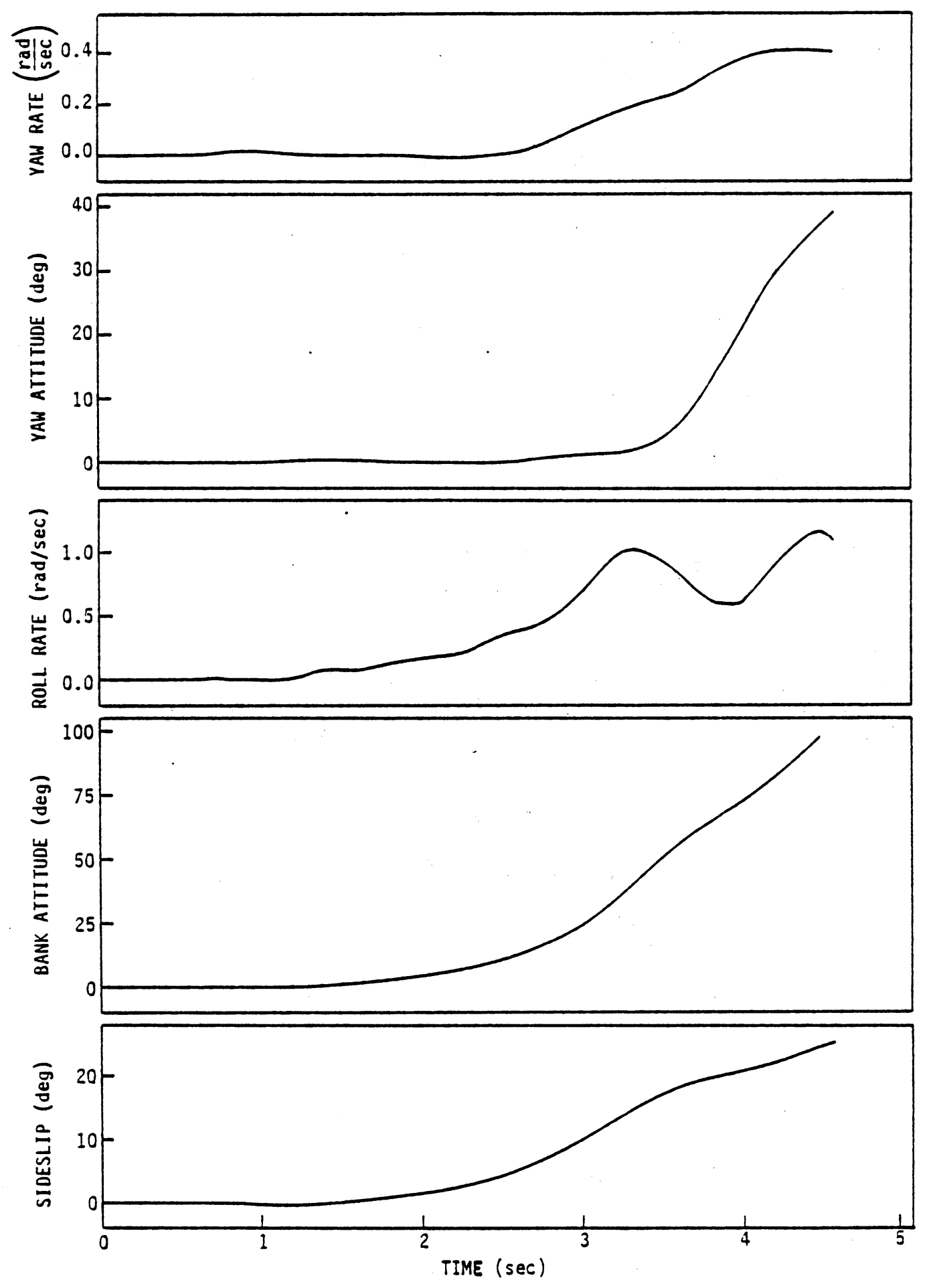

Figure $15 . b$ - SYMMETRIC STALL PENETRATION

$$
\text { Basic Wing - Lateral Parameters }
$$

Asymmetric Induced Angle-of-Attack Solution 


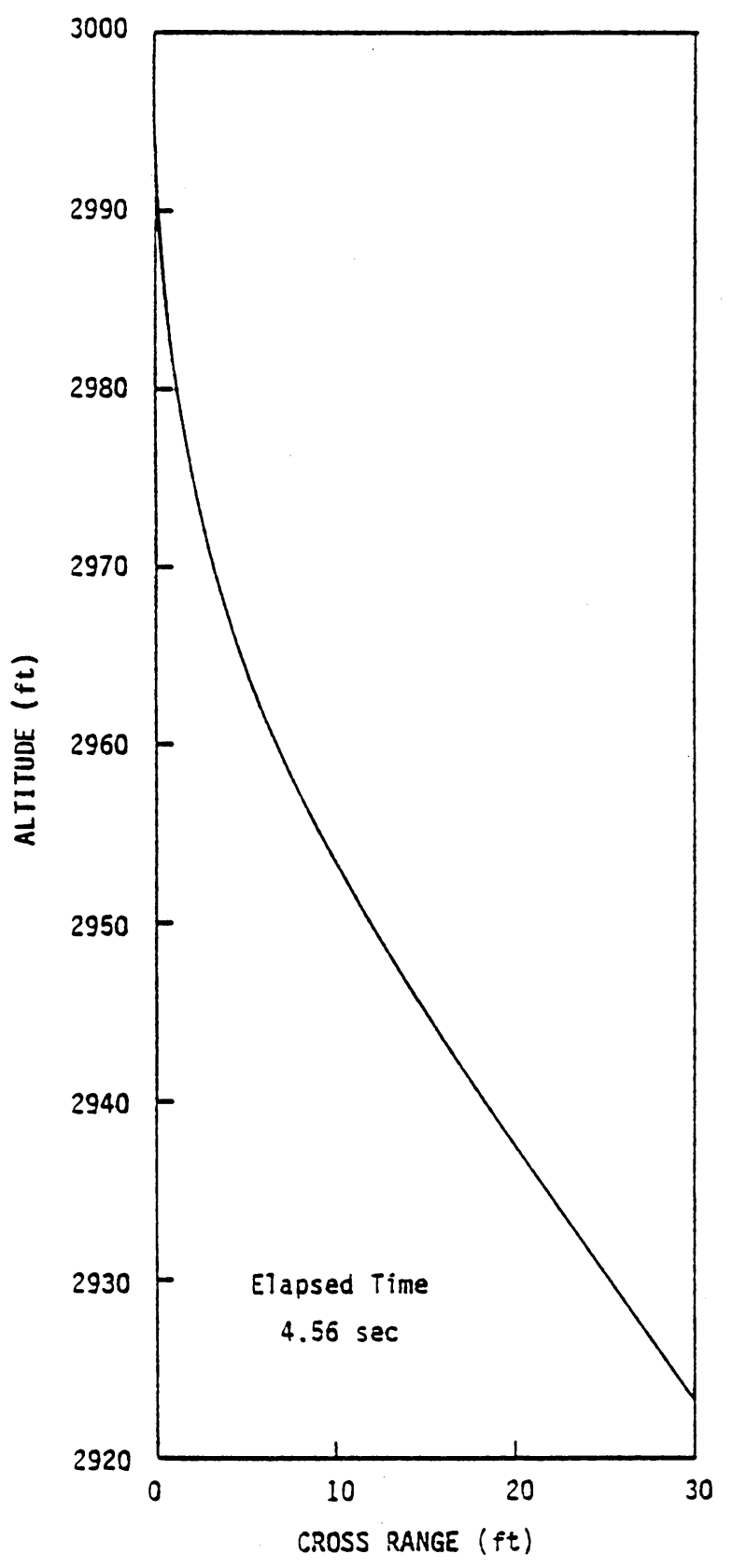

Figure 15.C - SYMMETRIC STALL PENETRATION Basic Wing - Altitude vs Cross Range Plot Asymmetric Induced Angle-of-Attack Solution 

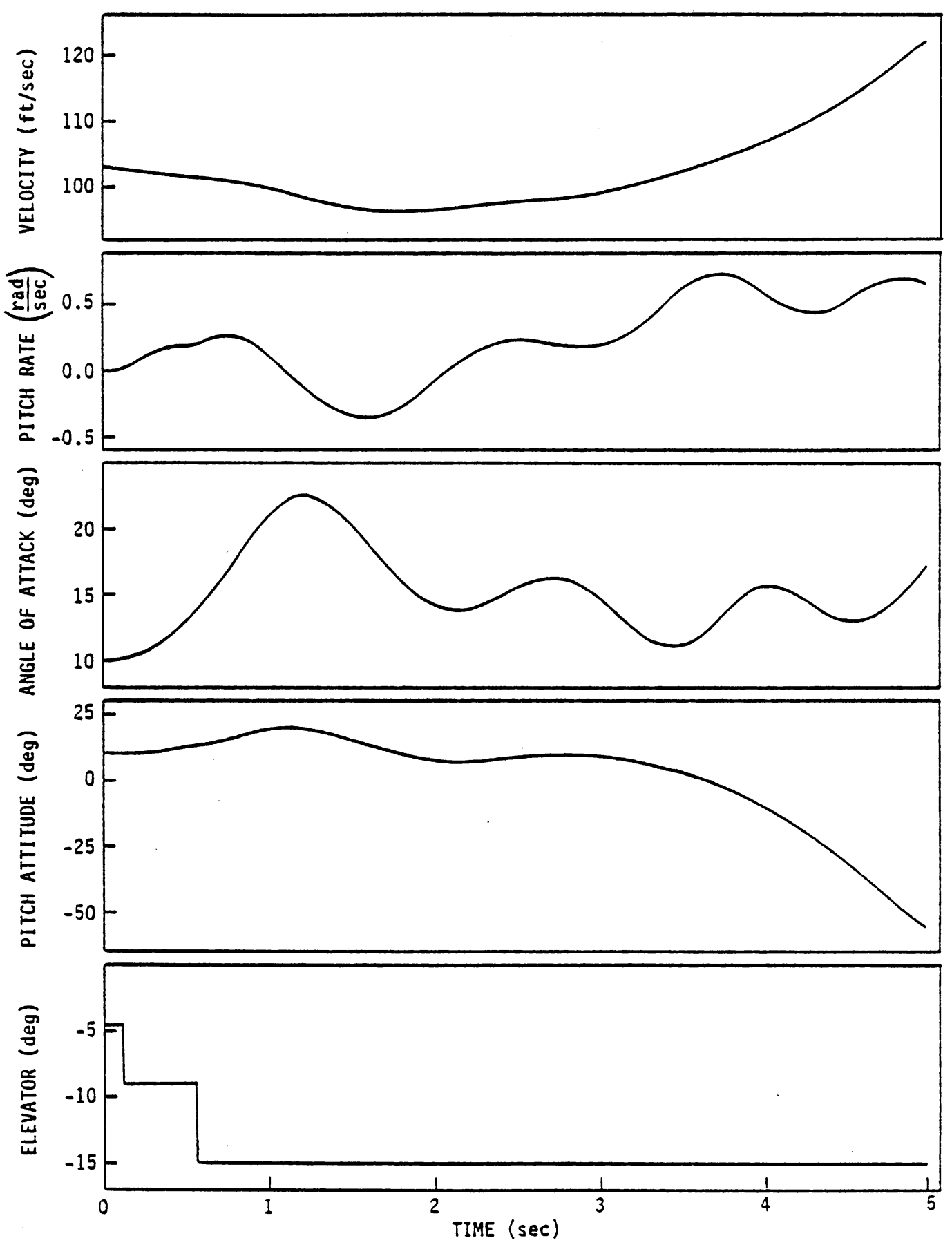

Figure 16.a - ASYMMETRIC STALL PENETRATION

Basic Wing - Longitudinal Parameters

Initial Sideslip Angle $=-5^{\circ}$ 
91
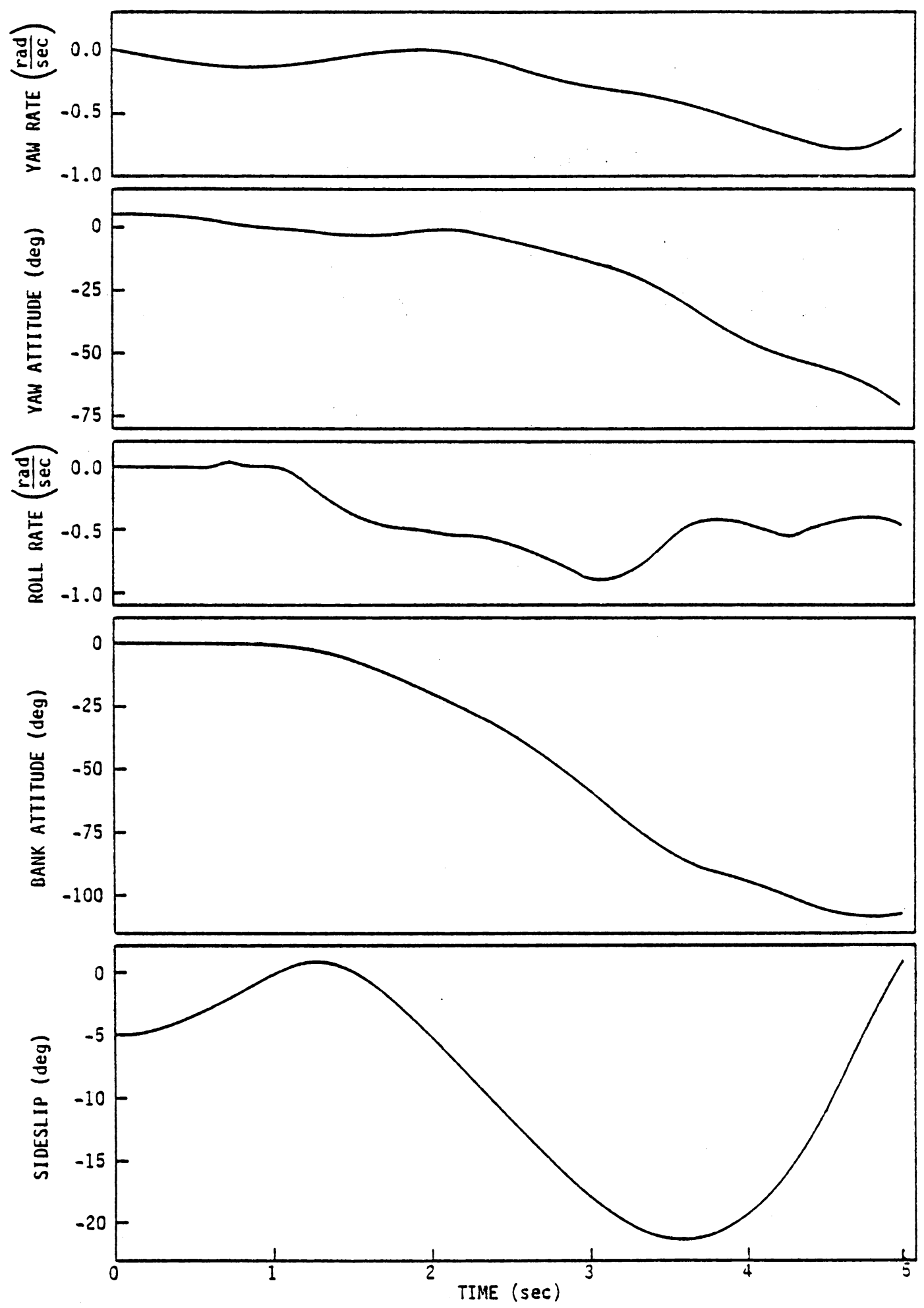

Figure 16.b - ASYMMETRIC STALL PENETRATION

Basic Wing - Lateral Parameters

Initial Sideslip Angle $=-5^{\circ}$ 


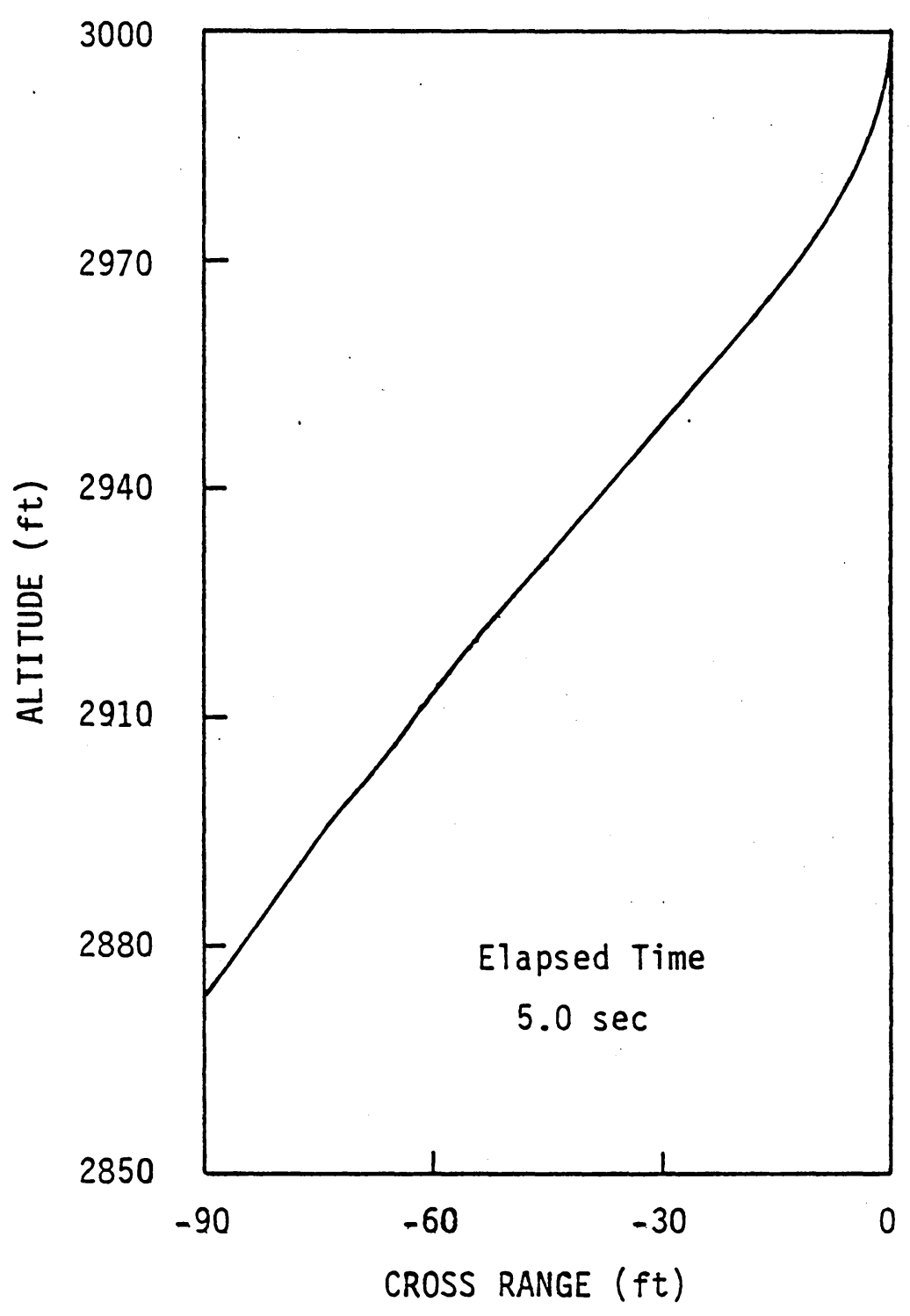

Figure 16.C - ASYMMETRIC STALL PENETRATION Basic Wing - Altitude vs Cross Range Plot Initial Sideslip Angle $=-5^{\circ}$ 


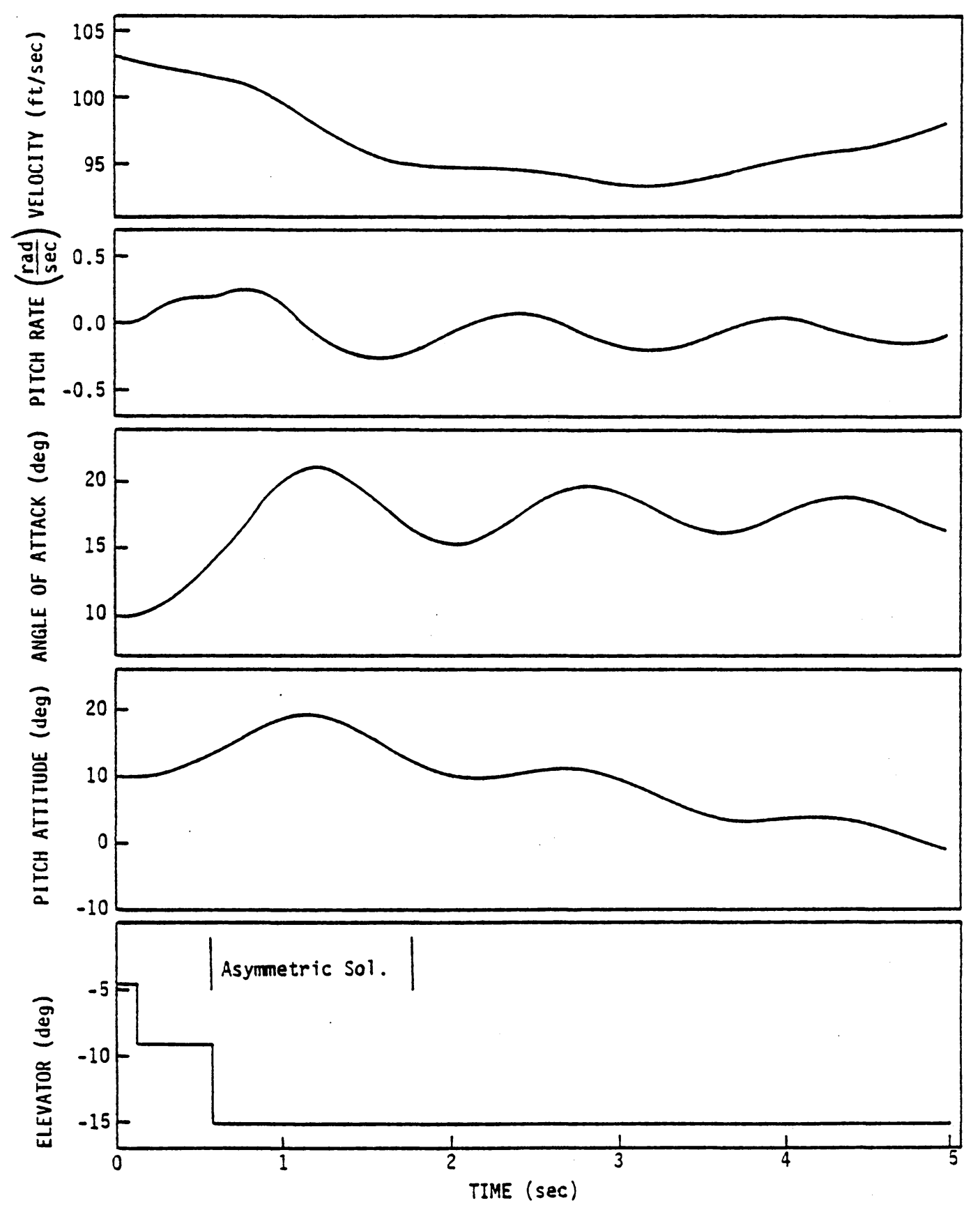

Figure 17.a - SYMMETRIC STALL PENETRATION Outboard Drooped Leading Edge - Longitudinal Parameters Asymmetric Induced Angle-of-Attack Solution 

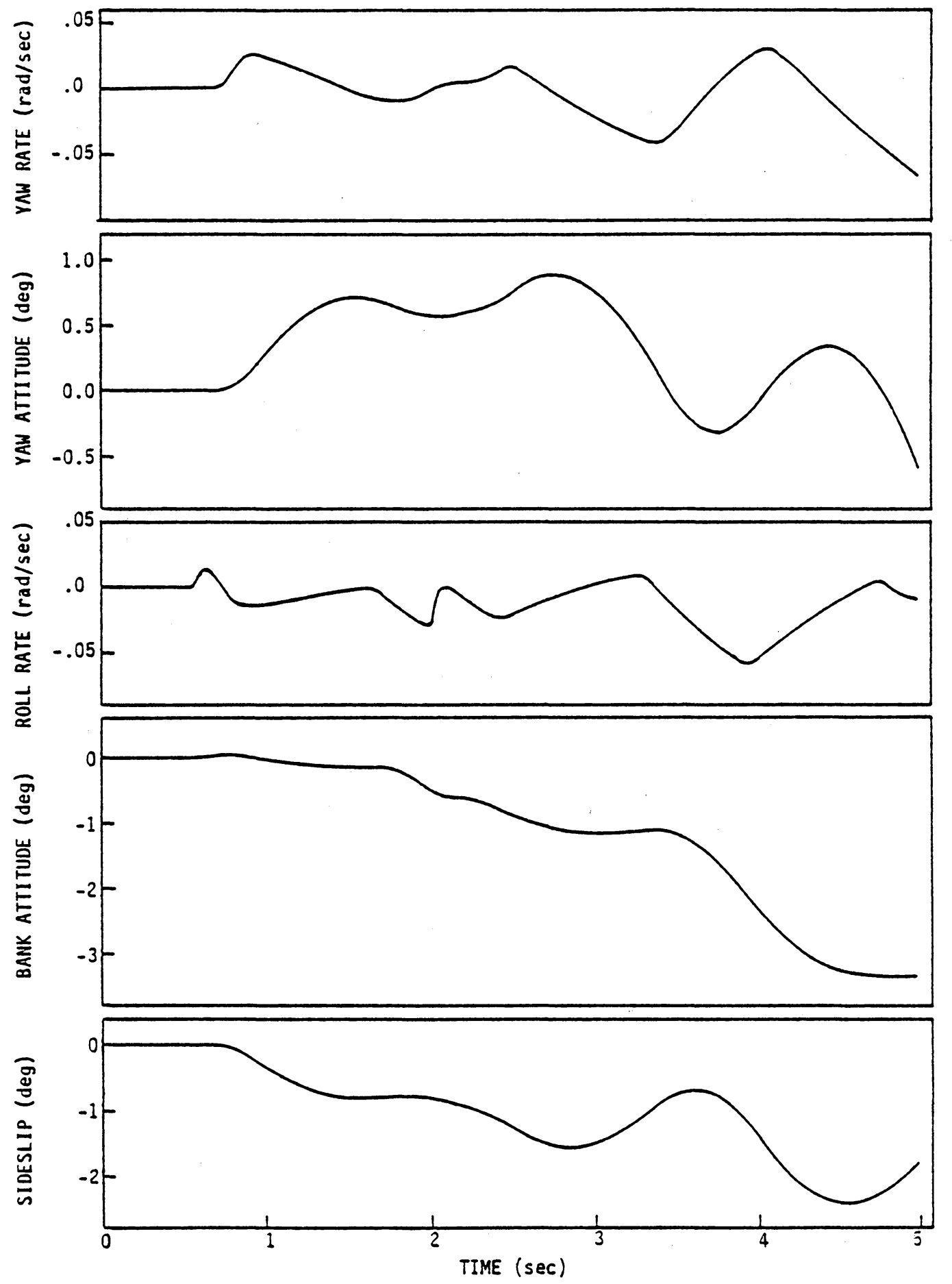

Figure $17 . b$ - SYMMETRIC STALL PENETRATION

Outboard Drooped Leading Edge - Lateral Parameters

Asymmetric Induced Angle-of-Attack Solution 


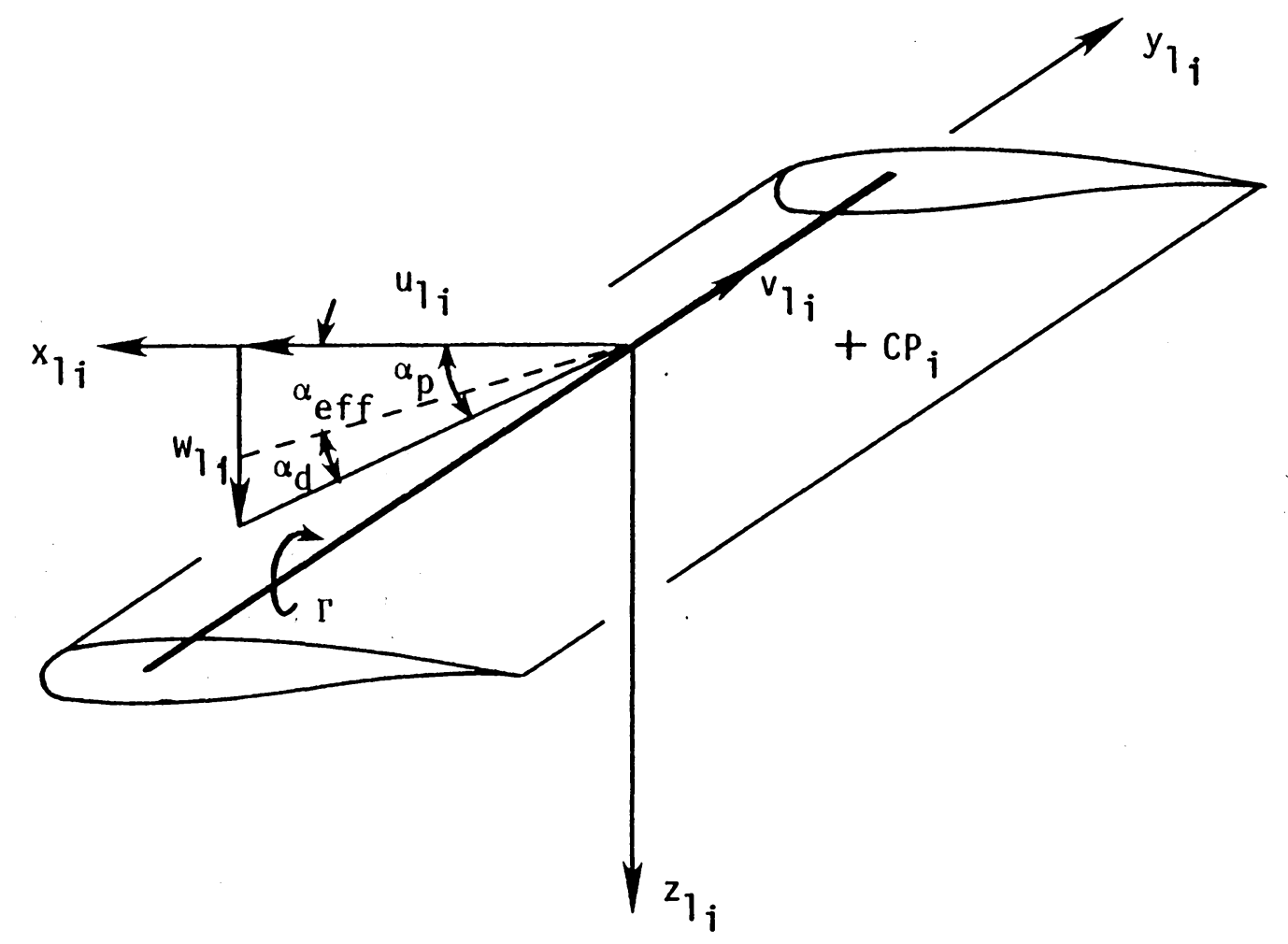

Figure C.1 - Local Wing Station $i$ Geometry for Determining Angles of Attack 


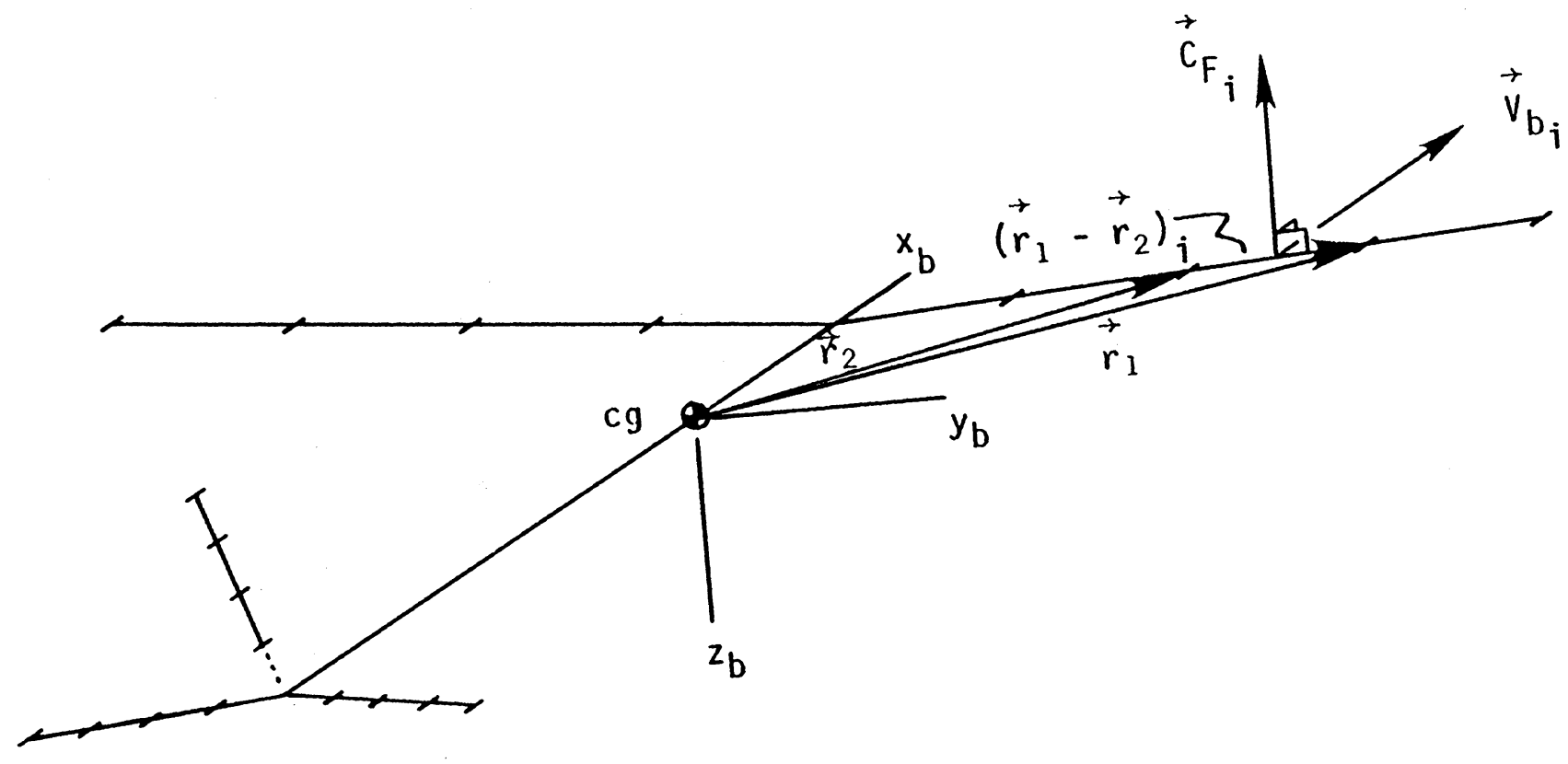

Figure C.2 - Geometry for Computing Local Force Coefficient $\vec{C}_{F_{j}}$ in Terms of Body-Axis System Vectors 


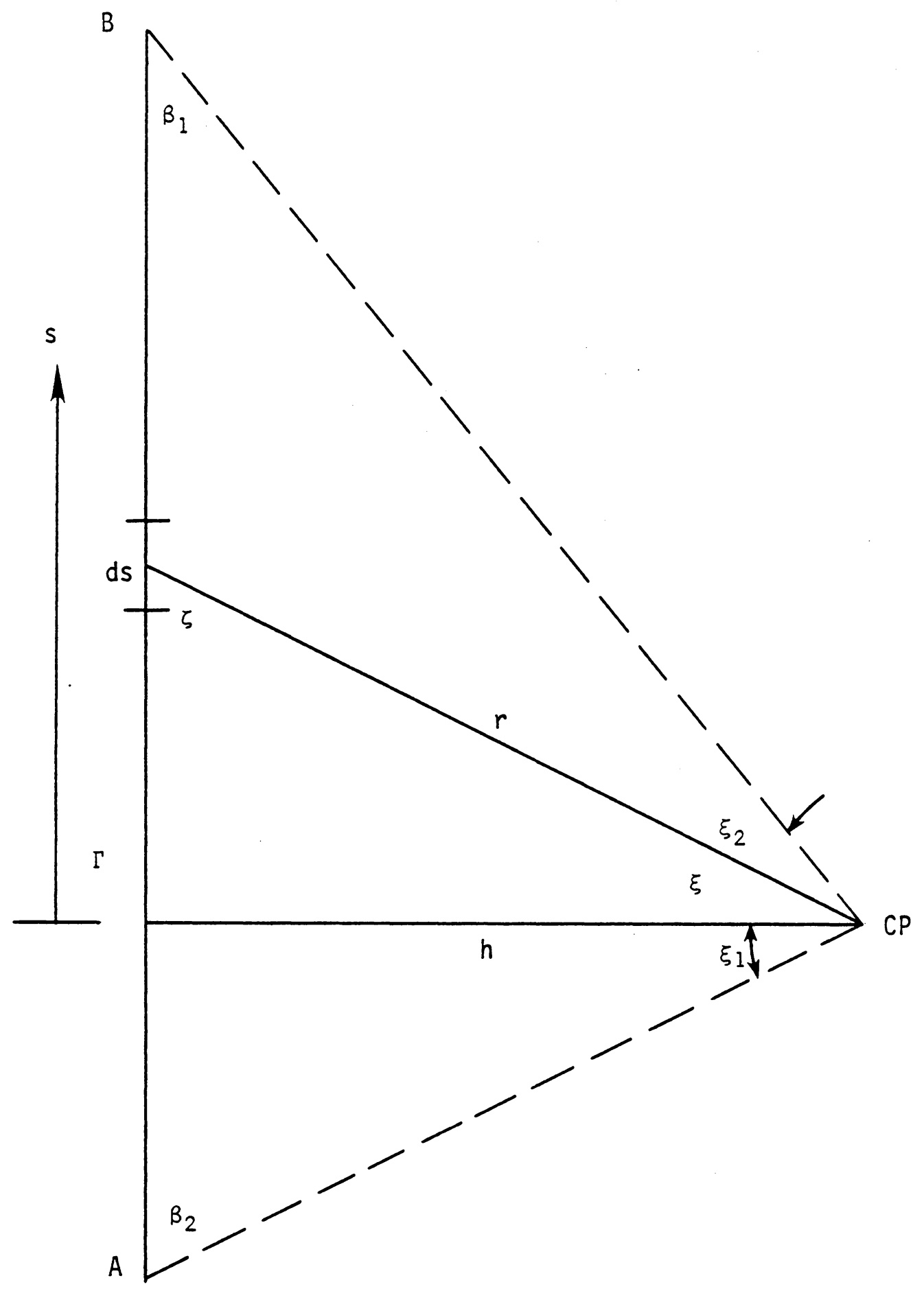

Figure D.1 - Geometry Used in Deriving the Velocity Induced at Point $C P$ by a Straight Vortex Filament 


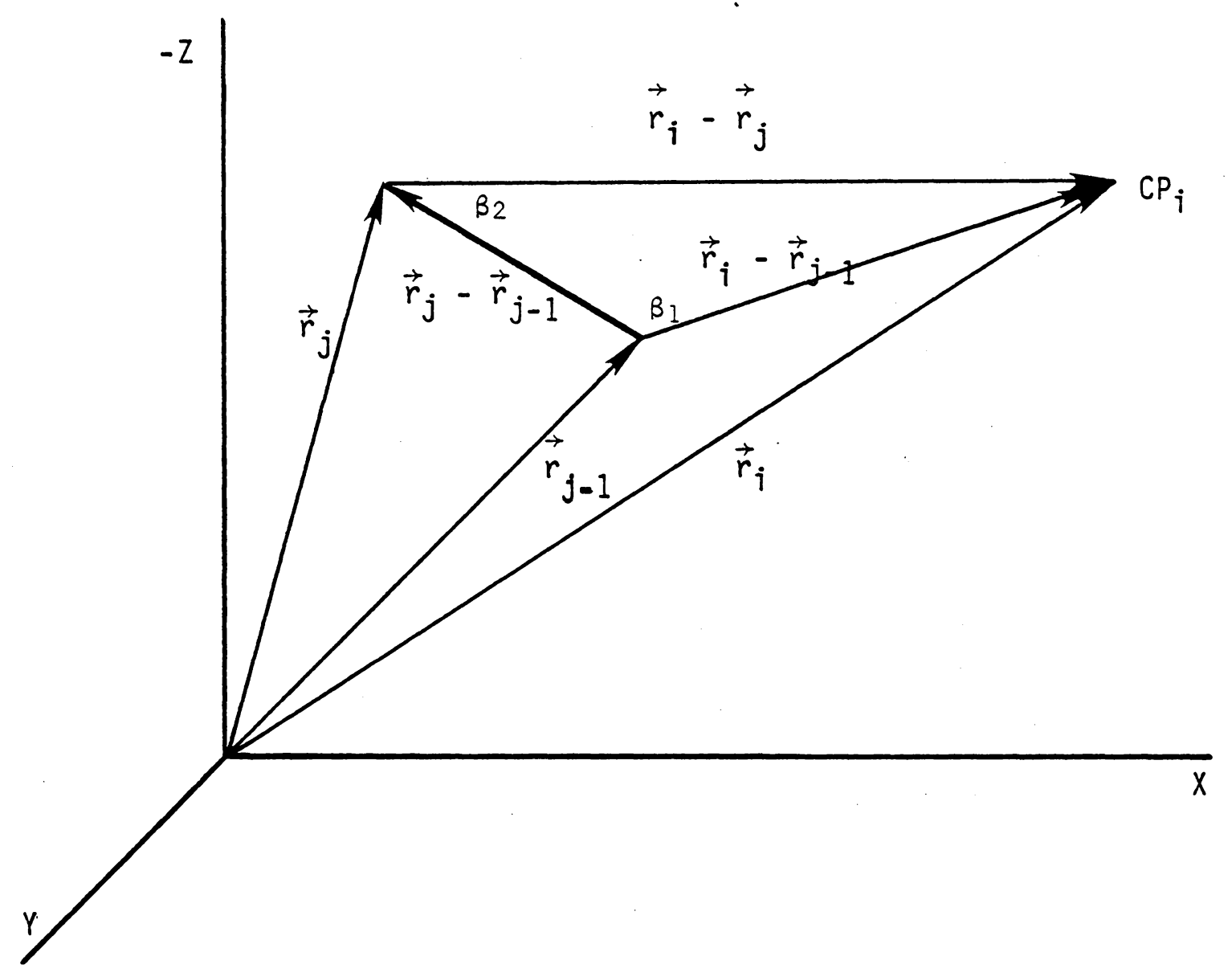

Figure 0.2 - Vector Relationship between a Vortex Element $j$ and Control Point $\mathfrak{i}$ in Inertial Space 


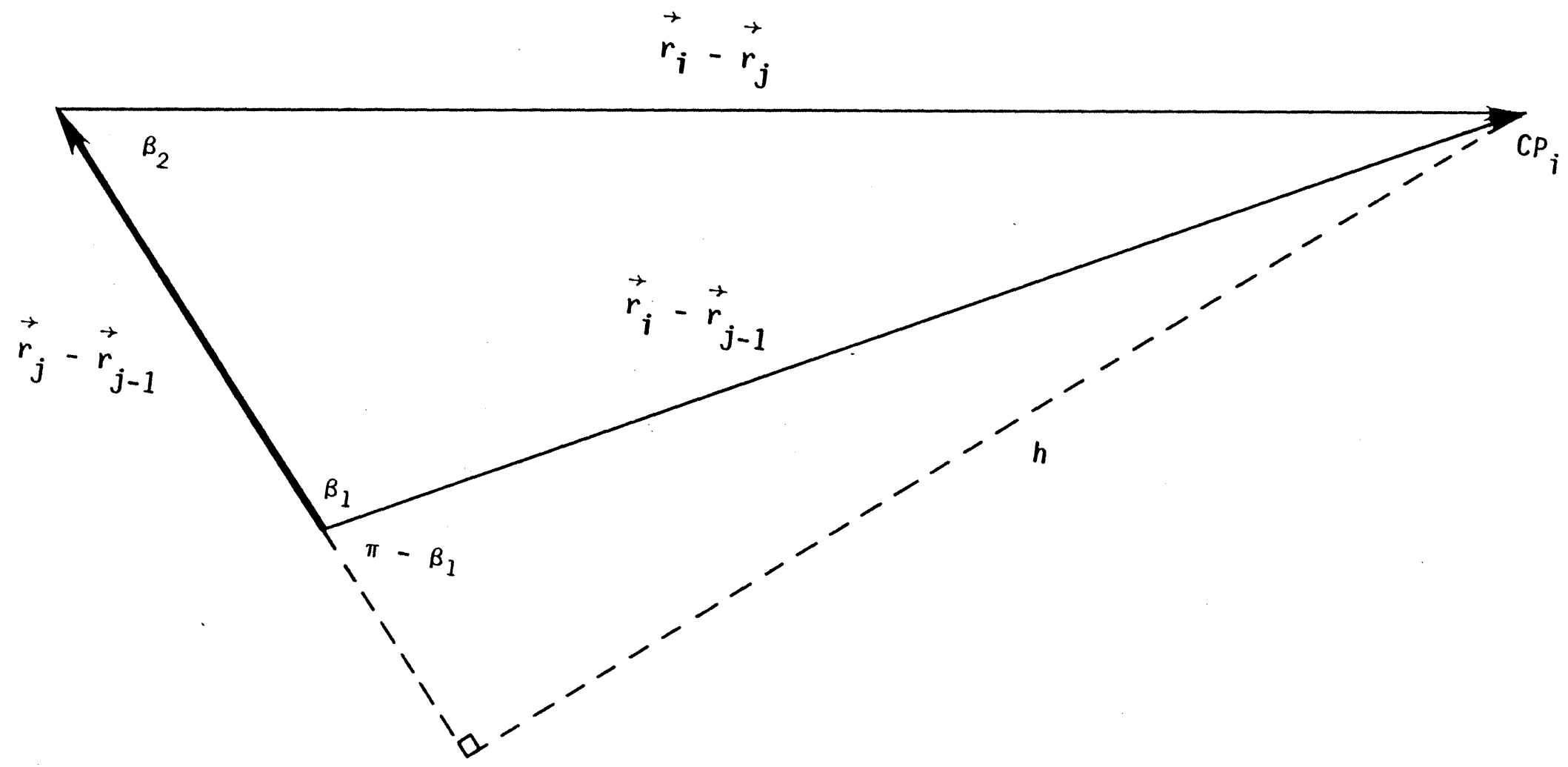

Figure D.3 - Inertial Space Plane Geometry for Computing the Scalar $h$
$h=$ Perpendicular Distance from Vortex Element $j$

$h$ - Perpendicular Distance from Vortex Element $j$ to Control Point $i$ 


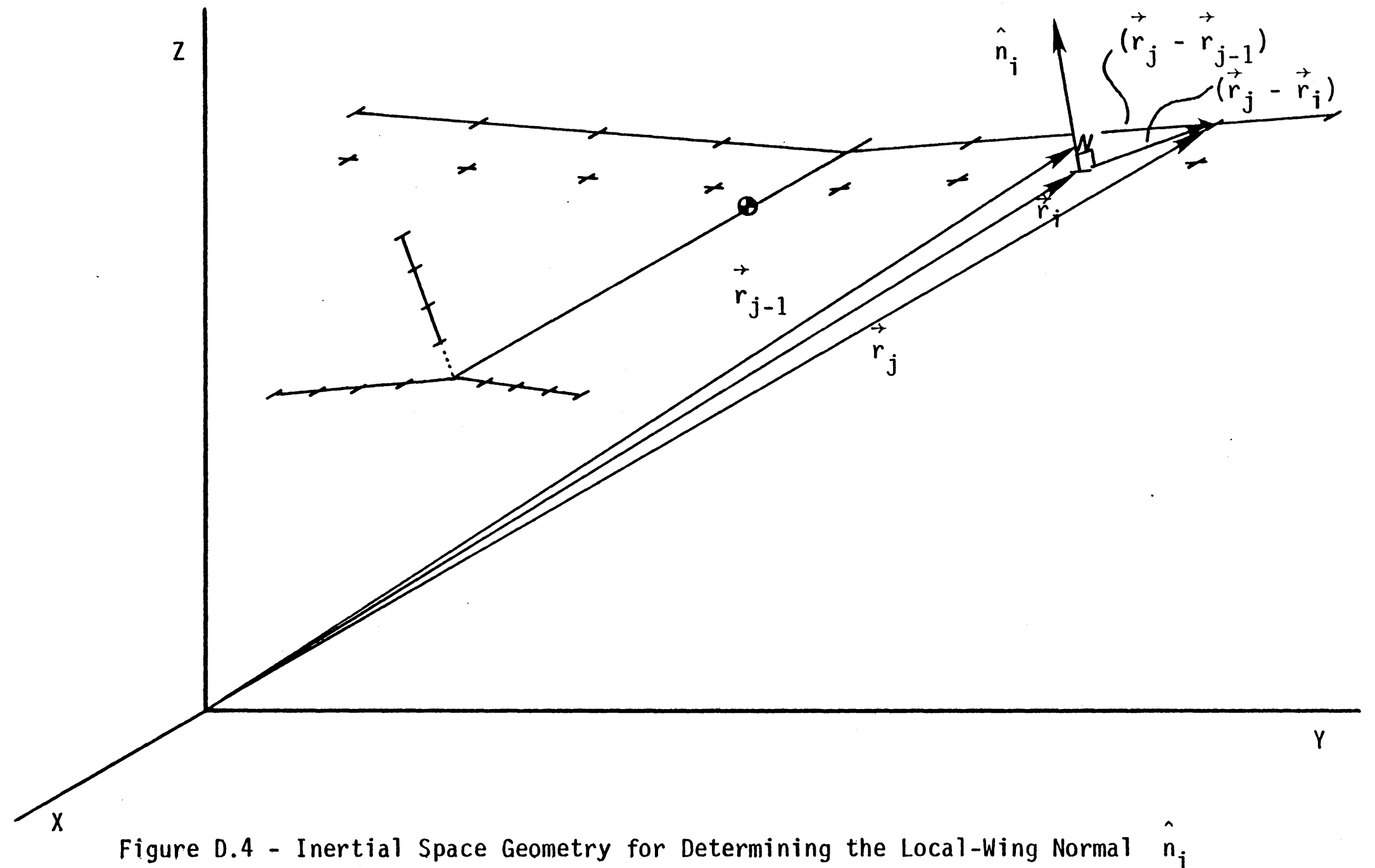




\section{APPENDIX A}

\section{EQUATIONS OF MOTION}

Below, the general equations of motion are written for atmospheric flight of a rigid-body aircraft. Excluded are engine gyroscopic effects, changing mass effects and vehicle products of inertia. The first three equations represent longitudinal motion; the next three govern lateral movement. Equations $A 7$ through $A 9$ are kinematic relations describing changes in the Euler orientation angles while the remainder are the flight trajectory equations. These 12 equations constitute the motion simulator of the dynamic model.

$m \dot{u}+m q w-m r v-x+m g \sin \theta-T=0$

$\dot{m} \dot{w}+m p v-m q u-Z-m g \cos \theta \cos \phi=0$

$I_{y} \dot{q}-\left(I_{z}-I_{x}\right) r p-M=0$

$m \dot{v}+m r u-m p w-Y-m g \cos \theta \sin \phi=0$

$I_{x} \dot{p}-\left(I_{y}-I_{z}\right) q r-M_{\ell}=0$

$I_{z} \dot{r}-\left(I_{x}-I_{y}\right) p q-N=0$

$\dot{\theta}-q \cos \phi+r \sin \phi=0$

$\dot{\phi}-p-q \sin \phi \tan \theta-r \cos \phi \tan \theta=0$

$\dot{\psi}-q \sin \phi \sec \theta-r \cos \phi \sec \theta=0$ 
$v_{x}=u \cos \theta \cos \psi+v(\sin \phi \sin \theta \cdot \cos \psi-\cos \phi \sin \psi)$

$+w(\cos \phi \sin \theta \cos \psi-\sin \phi \sin \psi)$

$v_{y}=u \cos \theta \sin \psi+v(\sin \phi \sin \theta \sin \psi+\cos \phi \cos \psi)$

$+w(\cos \phi \sin \theta \cdot \sin \psi-\sin \phi \cos \psi)$

(A11)

$v_{z}=-u \sin \theta+v \sin \phi \cos \theta+w \cos \phi \cos \theta$ 
APPENDIX B

\section{AXES TRANSFORMATIONS}

The transformation of the body-axis system into a local wing system can be accomplished by making three successive rotations about various axes. These rotations must be described in the particular order resembling how a physical wing is formed and mated to the fuselage. Here, it is assumed that a wing segment is rotated in the following order before being attached to the body:

(1) Rotate dihedral angle $n$ about $x_{b}$ axis to the system $x^{\prime} y^{\prime} z^{\prime}$

(2) Rotate sweep angle $\Lambda$ about $z^{\prime}$ axis to the system $x^{\prime \prime} y^{\prime \prime} z^{\prime \prime}$

(3) Rotate incidence angle $v$ about $y^{\prime \prime}$ axis to the system $x_{7} y_{1} z_{1}$ where subscripts $b$ and 1 stand for body- and local wing-axis systems, respectively.

We seek the transformation matrix required to express a body-axis vector $\vec{A}$, say, in the local wing system.

$$
\vec{A}_{1}=L_{1 b} \vec{A}_{b}
$$

This matrix $L_{1 b}$ can be developed by successive applications of elementary rotation matrices governed by the three sequential rotations above.

$$
L_{1 b}=\left[v_{y}\right]\left[\Lambda_{z}\right]\left[n_{x}\right]
$$


Expanding the single rotation matrices, we have

$$
L_{b}=\left[\begin{array}{ccc}
\cos \nu & 0 & -\sin \nu \\
0 & 1 & 0 \\
\sin \nu & 0 & \cos \nu
\end{array}\right]\left[\begin{array}{ccc}
\cos \Lambda & \sin \Lambda & 0 \\
-\sin \Lambda & \cos \Lambda & 0 \\
0 & 0 & 1
\end{array}\right]\left[\begin{array}{ccc}
1 & 0 & 0 \\
0 & \cos n & \sin n \\
0 & -\sin n & \cos n
\end{array}\right]
$$

Performing the matrix multiplications yields the desired transformation.

$$
L_{1 b}=\left[\begin{array}{ccc}
\cos \nu \cos \Lambda & \begin{array}{c}
\cos \nu \sin \Lambda \cos n \\
+\sin \nu \sin n
\end{array} & \begin{array}{c}
\cos \nu \sin \Lambda \sin n \\
-\sin \nu \cos n
\end{array} \\
-\sin \Lambda & \cos \Lambda \cos n & \cos \Lambda \sin n \\
\sin \nu \cos \Lambda & \sin \nu \sin \Lambda \cos n & \sin \nu \sin \Lambda \sin n \\
& -\cos \nu \sin n & +\cos \nu \cos n
\end{array}\right]
$$

For a left wing local coordinate system transformation, Equation B4 is used with a negative sweep angle while a negative dihedral angle is substituted for the right wing case. The parameters defining the transformation from the body to a local fin station are

$$
\eta=-90^{\circ}, \Lambda=\text { fin sweep angle, } \nu=0^{\circ}
$$

The inverse transformation (local-wing to body) is used in force resolution and is given by the transpose of the matrix above.

$$
L_{b 1}=L_{1 b}^{-1}=L_{1 b}^{T}
$$

The matrix associated with the transformation of body axes into inertial (local-horizontal) axes is developed similarly. The rotation angles are the Euler orientation angles and the sequence of rotations is 
(1) Rotate bank angle $-\phi$ about $x_{b}$ axis to the system $x^{\prime} y^{\prime} z^{\prime}$

(2) Rotate pitch angle $-\theta$ about $y^{\prime}$ axis to the system $x^{\prime \prime} y^{\prime \prime} z "$

(3) Rotate yaw angle $-\psi$ about $z^{\prime \prime}$ axis to the system $x_{h} y_{h} z_{h}$

where subscripts $b$ and $h$ denote body and horizontal coordinate systems respectively. The transformation matrix is

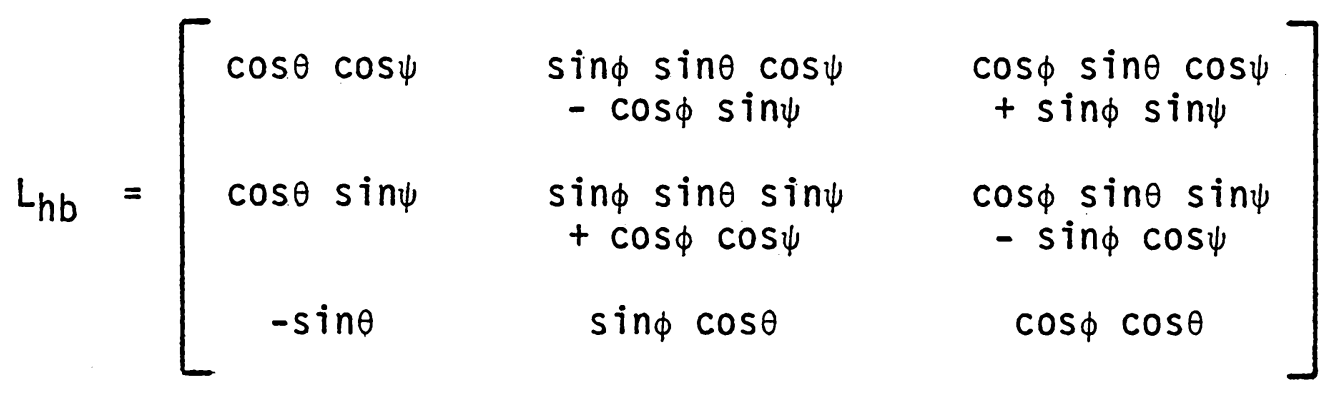

Note that the aircraft's velocity vector in inertial space (i.e. its trajectory) can be represented in terms of the body velocity components $(u, v, w)$ through this matrix. The resulting expressions are given in Appendix $A$ as Equations $A 10$ - A12. Also, the orientation of the vehicle in the inertial-axis system is obtained by transforming every body-axis vector defining a point of the airframe's geometry through the matrix $L_{h b}$. The position of every such geometric point with respect to the inertial origin is found by adding to the transformed vectors the space location of the center of gravity. 


\section{APPENDIX C \\ LOCAL ANGLE OF ATTACK DEFINITION AND FORCE RESOLUTION}

The nonlinear lifting line theory assumes that a given lifting surface can be segmented into discrete wing elements, each modeled by a bound lifting line filament and a control point at which the effective angle of attack is computed. Each spanwise wing section is assumed to possess the lift characteristics of a 20 airfoil. The two-dimensional properties of each section are further exploited in defining the local pitch angle of attack and resolving the local force acting on the bound vortex.

Consider the local wing station $i$ pictured in Figure C.1. The mean pitch angle of attack is computed at the section midpoint of the bound vortex segment. Let the location of this point be denoted by the vector $\vec{r}_{\mathrm{cg}_{i}}$ emanating from the body-axis system origin at the aircraft center of gravity. At any particular time when the pitch angle of attack $\alpha_{p_{j}}$ is to be determined, the airplane's velocity $\vec{v}_{c g}$ and total angular rate $\vec{\omega}_{c g}$ are known. (The components of are of course roll, pitch and yaw.) The local velocity $\vec{v}_{b_{i}}$ in the body-axis system is given by the rigid-body motion equation

$$
\vec{v}_{b_{i}}=\vec{v}_{c g}+\vec{\omega}_{c g} \times \vec{r}_{c g_{i}}
$$

This velocity vector is then transformed into the local-wing axis components $\vec{v}_{1_{i}}$ using the transformation matrix given by Equation B4. 


$$
\vec{v}_{1_{i}}=L_{1 b} \vec{V}_{b_{i}}
$$

The pitch angle of attack is now defined conventionally in terms of the local velocity components.

$$
\alpha_{p_{i}}=\arctan \left\{\frac{w_{1}}{u_{1}}\right\}_{i}
$$

The velocity quantity which occurs in the two-dimensional and threedimensional induced angle-of-attack definitions is the local $u_{1}$ resulting from the (C2) transformation. (See Equations 3,4,5 of Chapter III.)

The velocity term appearing in the Kutta-Joukowski Law $\left(V_{N_{j}}\right.$ in Equations 6 and 7 of Chapter III) is the local velocity component normal to the lifting line segment. This quantity is readily calculated by the expression

$$
v_{N_{i}}=\sqrt{u_{i}{ }^{2}+w_{1_{i}}^{2}}
$$

The third component $v_{1}$ lies along the bound vortex segment as shown in Figure C.1.

The direction of the local force experienced by the vortex element due to its "flight" is computed and resolved in the body-axis system after the downwash calculations have produced a converged induced angle of attack $\alpha_{d_{j}}$. In keeping with classical lifting line theory, the force is considered to be perpendicular to the plane formed by the vortex segment and the velocity vector associated with the effective angle of attack. The force is assumed to act at the bound vortex midpoint, 
denoted $\vec{r}_{c g_{i}}$ above. The local velocity components $\left(u_{7}, v_{7}, w_{1}\right)_{i}$ are modified to reflect airfoil section operation at the effective angle of attack and become the so-called relative wind components.

$$
\left(u_{1}, v_{1}, w_{1}\right)_{i} \rightarrow\left(u_{1}, v_{1}, w_{1}-u_{1} \tan \alpha_{d}\right)_{i}
$$

Represent the relative wind velocity vector as $\vec{V}^{\prime}{ }_{1_{i}}$ and transform to body axes components through the inverse of the matrix given by Equation B4.

$$
\vec{v}_{b_{i}}=L_{b l} \vec{V}^{\prime} l_{i}
$$

Now, the bound vortex segment may be represented as a vector whose direction is given by the sense of circulation according to the right hand rule. The head of this vector may be located with respect to the body-axis origin by the vector $\vec{r}_{1}$ and its tail by $\vec{r}_{2}$. (See Figure C.2.) This vortex vector is described by the vector difference $\left(\vec{r}_{1}-\vec{r}_{2}\right)_{j}$. The direction $\vec{D}$ of the force experienced by the lifting line is given in body components by the cross product

$$
\vec{D}_{i}=\frac{\left(\vec{r}_{1}-\vec{r}_{2}\right)_{i} \times \vec{v}_{b_{i}}}{\left|\left(\vec{r}_{1}-\vec{r}_{2}\right)_{i} \times \vec{v}_{b_{i}}\right|}
$$

The force magnitude results from the nonlinear lifting line theory discussed in Chapter III and is given in two-dimensional coefficient form. Hence,

$$
\vec{C}_{F_{i}}=\vec{D}_{i} \cdot C_{L}\left(\alpha_{\text {eff }}\right)
$$


Since the wing (or tail or fin) is segmented into $N$ equal length vortex elements, the total wing force coefficient is

$$
\vec{C}_{F}=\frac{1}{N} \sum_{i}^{N} \vec{C}_{F_{i}}=\frac{1}{N} \sum_{i}^{N} \vec{D}_{i} \cdot C_{L}\left(\alpha_{\text {eff }}\right)
$$

The three components of Equation $C 9$ are the body-axis force coefficient $C_{X}, C_{Y}$ and $C_{Z}$ and may be used in the equations of motion. However, the coefficient $C_{X}$ may be a poor axial force estimate since no "fuselage profile" influence is included in the lifting line theory. The present model uses an axial force variation with angle of attack measured in static wind tunnel tests. (See Chapter IV.) The side and normal coefficients as calculated above are assumed to be good estimates of the body forces.

Since the sectional force coefficients $\vec{C}_{F_{j}}$ are assumed to act at the midpoints of the respective lifting line elements, $\vec{r}_{\mathrm{cg}}$, the body moment coefficients, roll, pitch and yaw, can be determined by summing the cross products of the individual forces with their positions relative to the body-axis origin. The relations are

$$
\begin{aligned}
& C_{\ell}=\frac{1}{N b} \sum_{i}^{N}\left(\vec{r}_{C_{C g}} \times \vec{C}_{F_{j}}\right) \cdot \hat{i} \\
& C_{M}=\frac{1}{N C} \sum_{i}^{N}\left(\vec{r}_{C g_{j}} \times \vec{C}_{F_{i}}\right) \cdot \hat{j} \\
& C_{N}=\frac{1}{N b} \sum_{i}^{N}\left(\vec{r}_{C g_{j}} \times \vec{C}_{F_{i}}\right) \cdot \hat{k}
\end{aligned}
$$


where the unit vectors along the body $x-, y$ - and $z$-axis are $\hat{i}, \hat{j}$ and $\hat{k}$ respectively.

of course all forces and moments must be dimensionalized from the coefficients with respect to the lifting surface (wing, tail or fin) from which they originate. The force and moment components from all surfaces are summed and input to the equations of motion. 
APPENDIX D

DOWNWASH CALCULATIONS OF VORTEX SYSTEM

The calculation of the downwash contribution at a particular control point due to a given vortex system element (bound or free) is presented in this Appendix in a general formulation. First, the Law of Biot and Savart is presented in an integrated form which expresses the downwash at a given location (the control point) due to any finite length straight vortex segment. This downwash is normal to the plane defined by the segment and the point. Next, the quantities appearing in the integrated expression are formulated in terms of vectors representing the relative positions of the vortex element and the control point. The fact that the three-dimensional wake geometry is recorded in inertial space renders this vector approach most applicable. Finally, a procedure for determining the component of downwash normal to the wing at the control point is necessary because of the three-dimensionality. For this sample calculation, the vortex segment is assumed to have unit circulation strength.

The Biot-Savart Law is an expression for the increment of velocity dw induced by a vortex filament element ds at the control point $C P$ (Fig. D.1).

$$
d w=\frac{1}{4 \pi} \frac{\sin \zeta d s}{r^{2}}
$$

$r$ is the distance between the ds segment of the filament and the point $C P$ and $\zeta$ is the angle between the length $r$ and the straight filament. 
The total downwash contribution due to a finite length of this filament (from point $A$ to point $B$ ) is found by integration.

$$
\Delta W=\int_{B^{\prime}}^{A^{3}} \frac{1}{4 \pi} \frac{\sin \zeta}{r^{2}} d s
$$

The following geometric definitions allow an integration variable transformation if we specify the perpendicular distance from the filament to $C P$ as $h$ and the angle between the lengths $r$ and $h$ as $\xi$ : (See Figure D.1.)

$$
\begin{aligned}
& r^{2}=h^{2}+s^{2}=h^{2}\left(1+s^{2} / h^{2}\right) \\
& \text { where } s / h=\tan \xi \text { or } s=h \tan \xi
\end{aligned}
$$

Differentiating,

$$
d s=h \sec ^{2} \xi d \xi
$$

Substituting Equation D4 into D3 we obtain

$$
\begin{gathered}
r^{2}=h^{2}\left(1+\tan ^{2} \xi\right)=h^{2} \sec ^{2} \xi \\
\sin \zeta=\frac{h}{r}=\cos \xi
\end{gathered}
$$

Now,

and the integral $\mathrm{D} 2$ becomes

$$
\begin{aligned}
& \Delta \mathrm{W}=\int_{\xi_{1}}^{\xi_{2}} \frac{1}{4 \pi} \frac{\cos \xi}{h^{2} \sec ^{2} \xi} h \sec ^{2} \xi d \xi \\
& \Delta \mathrm{W}=\frac{1}{4 \pi h} \int_{\xi_{1}}^{\xi_{2}} \cos \xi \mathrm{d} \xi
\end{aligned}
$$

By the geometry of Figure D.1, we have $\xi_{2}=\pi / 2-\beta_{1}$ and

$$
\xi_{1}=\pi / 2-\beta_{2}
$$


Hence, $\begin{aligned} \Delta W & =\frac{1}{4 \pi h} \sin \xi \mid \begin{array}{c}\pi / 2-\beta_{1} \\ -\left(\pi / 2-\beta_{2}\right)\end{array} \\ \Delta W & =\frac{1}{4 \pi h}\left(\cos \beta_{1}+\cos \beta_{2}\right)\end{aligned}$

Equation $D 8$ is the integrated Biot-Savart Law yielding the downwash velocity at a point located a perpendicular distance $h$ from the vortex segment and induced by the straight length of filament included within the angles $B_{1}$ and $B_{2}$ measured with respect to the point. The quantities $h, \cos \beta_{1}$ and $\cos \beta_{2}$ are now expressed in terms of the vector location of a given vortex segment and control point in inertial space.

Let $i$ denote a particular control point on the wing at which the downwash $\Delta \mathrm{w}_{\mathbf{j}}$ is to be computed. At a given time, the control point's location in inertial space is specified by the vector $\vec{r}_{i}$. Let the vortex segment inducing the downwash velocity be represented by a vector whose direction is indicated by the sense of its circulation according to the right hand rule. The head of the vortex vector is located in the inertial system at the prescribed time by the vector $\vec{r}_{j}$; its tail is given by $\vec{r}_{j-1}$. This geometry is pictured in Figure D.2. The plane formed by the control point and the vortex filament is the same as shown in Figure D.1.

The vector from the vortex element head to the control point is $\vec{r}_{i}-\vec{r}_{j}$ while the corresponding vector emanating from the vortex tail is $\vec{r}_{i}-\vec{r}_{j-1}$. The vortex vector itself is defined by the vector 
difference $\vec{r}_{j}-\vec{r}_{j-1}$. An expression for the term $\cos \beta_{1}$ can be determined from these vector quantities by writing the vector dot product for $\vec{r}_{j}-\vec{r}_{j-1}$ and $\vec{r}_{i}-\vec{r}_{j-1}$.

$\left(\vec{r}_{j}-\vec{r}_{j-1}\right) \cdot\left(\vec{r}_{i}-\vec{r}_{j-1}\right)=\left|\vec{r}_{j}-\vec{r}_{j-1}\right|\left|\vec{r}_{i}-\vec{r}_{j-1}\right| \cos \beta_{1}$

Solving for $\cos \beta_{1}$

$$
\cos \beta_{1}=\frac{\left(\vec{r}_{j}-\vec{r}_{j-1}\right) \cdot\left(\vec{r}_{i}-\vec{r}_{j-1}\right)}{\left|\vec{r}_{j}-\vec{r}_{j-1}\right|\left|\vec{r}_{i}-\vec{r}_{j-1}\right|}
$$

An expression for $\cos \beta_{2}$ can be similarly derived from consideration of the vectors $\vec{r}_{i}-\vec{r}_{j}$ and $\vec{r}_{j-1}-\vec{r}_{j}$. Note the change in direction of the vortex vector representation. This is necessary for proper quadrant definition of the angle $\beta_{2}$. The result is

$$
\cos \beta_{2}=\frac{\left(\vec{r}_{j-1}-\vec{r}_{j}\right) \cdot\left(\vec{r}_{i}-\vec{r}_{j}\right)}{\left|\vec{r}_{j-1}-\vec{r}_{j}\right|\left|\vec{r}_{i}-\vec{r}_{j}\right|}
$$

Since $h$ is the perpendicular distance from the vortex segment to the control point, its vector-based definition is readily found from the plane geometry indicated in Figure D.3. From the right triangle relations,

$h=\left|\vec{r}_{i}-\vec{r}_{j-1}\right| \sin \left(\pi-\beta_{1}\right)=\left|\vec{r}_{i}-\vec{r}_{j-1}\right| \sin \beta_{1}$

The vector cross product for $\vec{r}_{j}-\vec{r}_{j-1}$ and $\vec{r}_{i}-\vec{r}_{j-1}$ is $\left|\left(\vec{r}_{j}-\vec{r}_{j-1}\right) \times\left(\vec{r}_{i}-\vec{r}_{j-1}\right)\right|=\left|\vec{r}_{j}-\vec{r}_{j-1}\right|\left|\vec{r}_{i}-\vec{r}_{j-1}\right| \sin \beta_{1}$ 
Substituting from Equation D12, and solving for $h$, yields

$$
h=\frac{\left|\left(\vec{r}_{j}-\vec{r}_{j-1}\right) \times\left(\vec{r}_{i}-\vec{r}_{j-1}\right)\right|}{\left|\vec{r}_{j}-\vec{r}_{j-1}\right|}
$$

Note that the direction of the induced velocity at the control point is normal to the plane specified by the vortex vector and $C P$. This is the same direction obtained by crossing the vortex vector into the $\left(\vec{r}_{i}-\vec{r}_{j-1}\right)$ vector. Hence the direction of the downwash in inertial space is given by the unit vector

$$
\hat{u}_{i}=\frac{\left(\vec{r}_{j}-\vec{r}_{j-1}\right) \times\left(\vec{r}_{i}-\vec{r}_{j-1}\right)}{\left|\left(\vec{r}_{j}-\vec{r}_{j-1}\right) \times\left(\vec{r}_{i}-\vec{r}_{j-1}\right)\right|}
$$

However, it is the component of the downwash normal to the wing at the control point which is required for the induced angle-of-attack calculations. The normal direction to the wing may be found by crossing any two vectors lying in the plane of the wing. In the inertial space of Figure 0.4 , the vectors $\vec{r}_{j-1}$ and $\vec{r}_{j}$ locate the tail and head of a bound vortex vector $\vec{r}_{j}-\vec{r}_{j-1}$ positioned at the wing quarter chord. The control point at the three-quarter chord is specified by the vector $\vec{r}_{i}$. The two in-the-plane-of-the-wing vectors are $\vec{r}_{j}-\vec{r}_{i}$ and $\vec{r}_{j}-\vec{r}_{j-1}$. The unit normal to the wing is then given by

$$
\hat{n}_{i}=\frac{\left(\vec{r}_{j}-\vec{r}_{j-1}\right) \times\left(\vec{r}_{j}-\vec{r}_{i}\right)}{\left|\left(\vec{r}_{j}-\vec{r}_{j-1}\right) \times\left(\vec{r}_{j}-\vec{r}_{i}\right)\right|}
$$


Finally, the normal component of downwash is given by

$$
\Delta w_{i_{n}}=\left(\hat{n}_{i} \cdot \hat{u}_{i}\right) \cdot \Delta w
$$

In summary, the effective downwash at a particular control point induced by a vortex element (bound or free) of unit circulation strength is given by Equation 017 where $\Delta \mathrm{W}$ is the downwash magnitude developed in Equations D8, D10, D11, D14 and $\left(\hat{n}_{i}-\hat{u}_{i}\right)$ relates this downwash to the wing-normal component. The above calculations must be performed for all yortex system elements and the results summed to find the total downwash at each control point. 


\section{APPENDIX E}

\section{FORCED-ROLL OSCILLATION DATA REDUCTION}

Assume a small perturbation expansion is applicable to the total aerodynamic rolling moment coefficient $C_{\ell}$

$$
C_{\ell}=C_{\ell_{p}} \Delta p+C_{\ell_{\beta}} \Delta \beta+C_{\ell_{\dot{\beta}}} \dot{\Delta \beta}+\text { higher order time derivatives }
$$

In the absence of yaw angle relative to the freestream, the model's sideslip angle is expressed by the kinematic relation

$$
\Delta \beta=\Delta \phi \sin \alpha
$$

where $\Delta \phi$ is given by the bank attitude input function

$$
\Delta \phi=\Delta \phi_{\text {MAX }} \sin \omega t
$$

Differentiating Equation E2,

$$
\dot{\Delta \beta}=\dot{\Delta \phi} \sin \alpha=\Delta p \sin \alpha
$$

where the time derivative of the bank angle is expressed as the roll rate. Substituting Equations E2 and E4 into the truncated form of Equation E1, we have

$$
C_{\ell}=C_{\ell_{p}} \Delta p+C_{\ell_{\beta}} \Delta \phi \sin \alpha+C_{\ell_{\dot{\beta}}} \Delta p \sin \alpha
$$

Using E3

$$
C_{\ell}=\left(C_{\ell p}+C_{\ell_{\dot{\beta}}} \sin \alpha\right) \Delta p+C_{\ell_{\beta}} \Delta \phi_{\operatorname{MAX}} \sin \omega t \sin \alpha
$$


The roll rate is found by differentiating Equation E3

$$
\Delta \mathrm{p}=\dot{\Delta \phi}=\Delta \phi_{\text {MAX }} \omega \cos \omega t
$$

Substituting Equation E7 into Equation E6 yields an expression for the output signal $C_{\ell}$ :

$$
C_{\ell}=\left\{\left(C_{\ell_{p}}+C_{\ell_{\dot{\beta}}} \sin \alpha\right) \omega \cos \omega t+C_{\ell_{B}} \sin \alpha \sin \omega t\right\} \Delta \phi_{M A X}
$$

The component out of phase with the input signal (E3) is

$C_{\ell_{\text {OUT }}}=\left\{\left(C_{\ell_{p}}+C_{\ell_{\dot{\beta}}} \sin \alpha\right) \omega \cos ^{2} \omega t+C_{\ell_{\beta}} \sin \alpha \sin \omega t \cos \omega t\right\} \Delta \phi_{\text {MAX }}$

The average value of this component over one period is given by the integral

$$
\bar{c}_{\ell_{\text {OUT }}}+\frac{1}{T} \int_{t_{0}}^{t_{0}+T} c_{\ell_{\text {OUT }}} d t
$$

Substituting Equation Eg into Equation E10 and integrating yields

$$
\bar{c}_{\ell_{\text {OUT }}}=\frac{1}{2} \Delta \phi_{\text {MAX }} \omega\left(c_{\ell_{p}}+C_{\ell_{\dot{B}}} \sin \alpha\right)
$$

Solying for the dynamic stability parameter and nondimensionalizing according to standard convention, we obtain

$$
c_{\ell_{p}}+c_{\ell_{\dot{B}}} \sin \alpha=\frac{4 \bar{c}_{\ell \text { OUT }} V_{\infty}}{{ }_{\text {MAX }} \omega b}
$$




\section{The vita has been removed from the scanned document}




\title{
A DYNAMIC MODEL FOR AIRCRAFT POSTSTALL DEPARTURE
}

\author{
by
}

Mark Andrew Hreha

\section{(ABSTRACT)}

An engineering model designed for the analys is of high angle-ofattack flight characteristics is developed and applied to the problem of aircraft poststall departure. The model consists of an aerodynamics package used interactively with a six degree-of-freedom flight simulator. The aerodynamics are computed via a nonlinear lifting line theory with unsteady wake effects due to a discrete, nonplanar vortex system. A fully configured aircraft (main wing, horizontal tail and vertical fin) is mathematically constructed by modeling all lifting surfaces with bound, discrete vortex segments and associated control points; vehicle geometric influence on high angle-of-attack flight characteristics is included through complete variability in the relative locations, orientations and sizes of the flight surfaces. This aircraft model is "flown" through prescribed maneuvers by integrating the equations of motion. Selected results of trajectory simulations presented for a typical general aviation aircraft provide the following insights to wing-drop departure subsequent to sta 11.

The abruptness of poststal1 rol1-off depends on the presence of flight asymmetries at the stall break and the rate of stall penetration. Such out-of-trim flight conditions induce asymmetric wing panel unstall 
subsequent to deep sta11 penetration resulting in large wing-dropproducing rol1 moments. However, the abrupt departure from symmetric flight conditions is also found to be mathematically possible. This is a consequence of multiple lifting line solutions which exist for bound vortex systems assigned the 1 ift properties of airfoils having stall discontinuities. The dynamic model is well suited to the prediction of departure resistance benefits realized through passive aerodynamic modifications, for example, drooped leading edge outboard wing panels. The model can also be applied to the generation of dynamic stability derivatives by analytically simulating forced oscillation test procedures. 